University of Rhode Island

DigitalCommons@URI

Open Access Master's Theses

2018

\title{
A LINE ARRAY OF DIRECTIONAL HYDROPHONES FOR IMPROVED DETECTION OF EMERGENCY LOCATOR BEACONS
}

Elizabeth Ann Alvanas

University of Rhode Island, elizabeth.alvanas@cox.net

Follow this and additional works at: https://digitalcommons.uri.edu/theses

\section{Recommended Citation}

Alvanas, Elizabeth Ann, "A LINE ARRAY OF DIRECTIONAL HYDROPHONES FOR IMPROVED DETECTION OF EMERGENCY LOCATOR BEACONS" (2018). Open Access Master's Theses. Paper 1311.

https://digitalcommons.uri.edu/theses/1311

This Thesis is brought to you for free and open access by DigitalCommons@URI. It has been accepted for inclusion in Open Access Master's Theses by an authorized administrator of DigitalCommons@URI. For more information, please contact digitalcommons-group@uri.edu. 
A LINE ARRAY OF DIRECTIONAL HYDROPHONES

FOR IMPROVED DETECTION OF

EMERGENCY LOCATOR BEACONS

BY

ELIZABETH A. ALVANAS

A THESIS SUBMITTED IN PARTIAL FULFILLMENT OF THE

REQUIREMENTS FOR THE DEGREE OF

MASTER OF SCIENCE

IN

OCEAN ENGINEERING

UNIVERSITY OF RHODE ISLAND

2018 


\title{
MASTER OF SCIENCE THESIS
}

OF

\author{
ELIZABETH A. ALVANAS
}

\section{APPROVED:}

Thesis Committee:

Major Professor

Harold T. Vincent II

James Hu

Manbir Sodhi

Nasser H. Zawia

DEAN OF THE GRADUATE SCHOOL

UNIVERSITY OF RHODE ISLAND

2018 


\begin{abstract}
The U.S Navy’s system for locating black boxes from downed aircraft is the Towed Pinger Locator 25 (TPL-25). The TPL-25 is a kite-shaped fiberglass shell that is towed behind a surface vessel while its single omnidirectional hydrophone listens for the signal being transmitted from the emergency beacon on one of the aircraft's black boxes. Recovery of the black boxes is critical to an accident investigation yet the window in which to locate the black box is short, typically only 30 days, and the success of the TPL-25 is limited as evidenced by the unsuccessful search for Malaysia Airlines Flight 370 in 2014.

This thesis explores how replacing the TPL-25’s single omnidirectional hydrophone with a device that has an increased directivity index, specifically with a linear array of directional multimode hydrophones, can increase the detection range of the TPL-25.
\end{abstract}




\section{ACKNOWLEDGMENTS}

As my thesis work comes to an end, I would like to express my deepest gratitude to those who supported me throughout this journey.

To my major professor Dr. Harold Vincent who taught many of my courses, served as my advisor, and inspired me by his enthusiasm for acoustics.

To my professor Dr. Steven Crocker who gave me the idea for my initial study and later helped me when I was struggling with three-dimensional plots in MATLAB.

To the professors at the Naval Post Graduate School who taught my first graduate level courses and introduced me to acoustics.

To my colleagues at the Naval Undersea Warfare Center (NUWC), Newport, RI Mr. Anthony Paolero and Dr. Victor Evora for providing me access to their facilities, Mr. Jeffrey Szelag and Mr. Michael Warnock for their guidance in building my array, Mr. Michael Bergeron and Mr. Jason Burghouwt for their assistance in the Open Test Facility, Ms. Karen Villano who taught me how to solder, Dr. William Martin who graciously put my array design into SOLIDWORKS, and Dr. Stephen Butler, Dr. Benjamin Cray, Dr. Thomas Howarth and Dr. Andrew Hull for their willingness to answer my questions.

To the DeSantis Foundation for the generous donation that allowed me to purchase the materials I needed.

And finally, to my family - my husband Bruce, our children Kyle and Evan, and my parents Merrill and Pat Louks - for their patience, understanding and support throughout the many years it took me to complete my degree, and most importantly for always believing in me. To them, I dedicate this work. 


\section{TABLE OF CONTENTS}

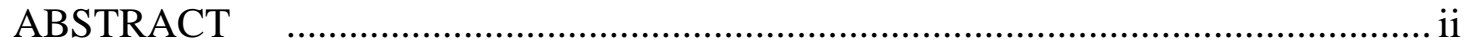

ACKNOWLEDGMENTS ............................................................................ ii

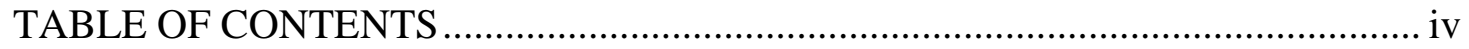

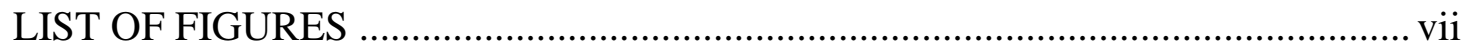

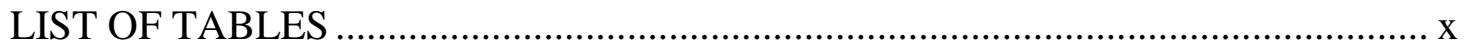

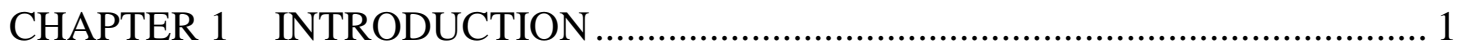

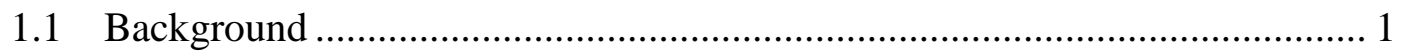

1.1.1 The Emergency Beacon’s Transmission Signal ...................................2

1.1.2 The Towed Pinger Locator 25 ...........................................................

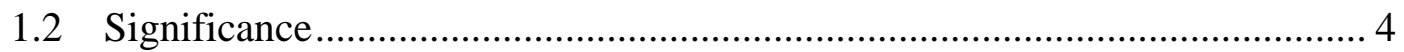

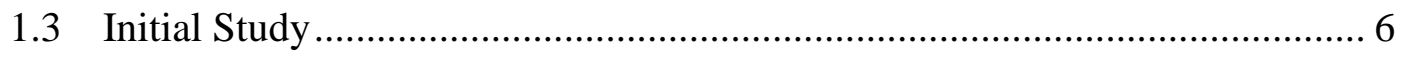

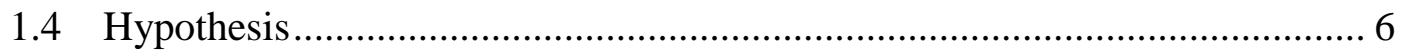

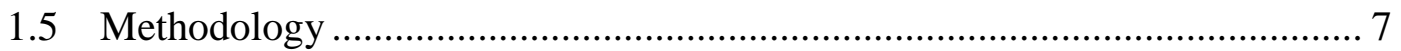

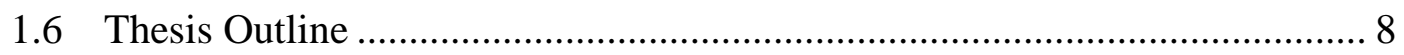

CHAPTER 2 TECHNOLOGY OVERVIEW ..................................................... 9

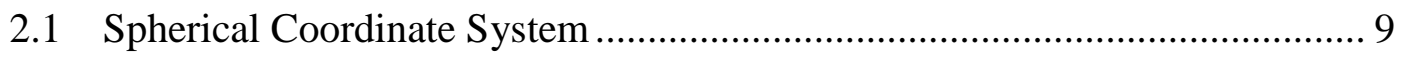

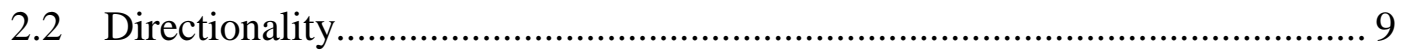

2.2.1 Beam Pattern.................................................................................. 10

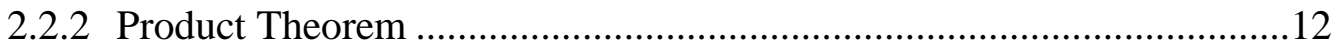

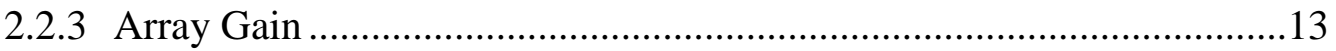

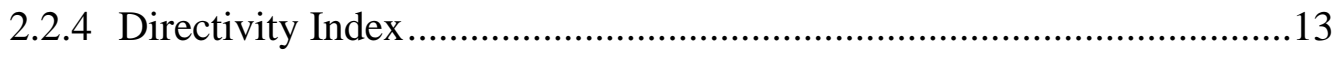

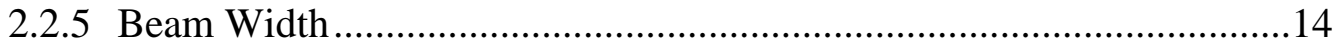


2.3 Multimode Transducer Technology ………………...................................... 15

2.3.1 Multimode Beam Patterns .......................................................................15

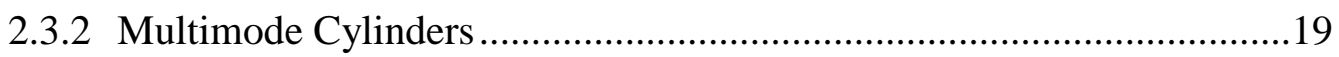

2.3.3 Multimode Signal Processing .................................................................23

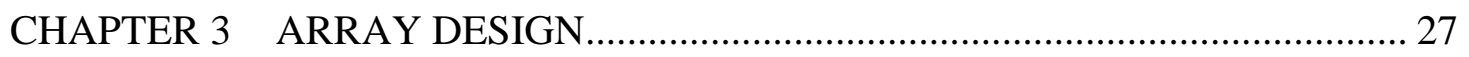

3.1 Budget Driven Decisions .......................................................................... 27

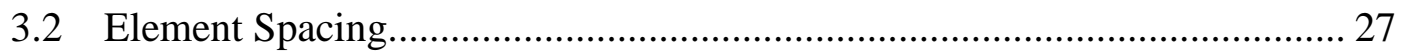

3.3 Final Array Design ................................................................................ 31

CHAPTER 4 THEORETICAL (PREDICTED) DIRECTIVITY ................................ 33

4.1 Single-Cylinder Multimode Prototype ............................................................. 33

4.2 Multimode Linear Hydrophone Array ……………........................................ 35

CHAPTER 5 IN-AIR CHARACTERIZATION .................................................... 38

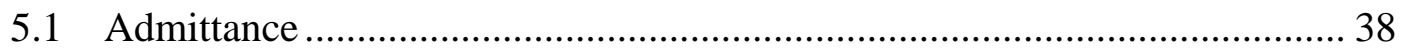

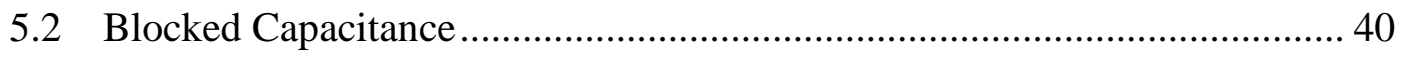

5.3 Leakage Resistance ................................................................................... 41

5.4 Dissipation Factor …………………………........................................... 42

CHAPTER 6 HYDROPHONE PROTOTYPE FABRICATION.............................. 44

CHAPTER 7 HYDROPHONE PROTOTYPE TESTING ………….......................... 46

7.1 Test Facility ............................................................................................. 46

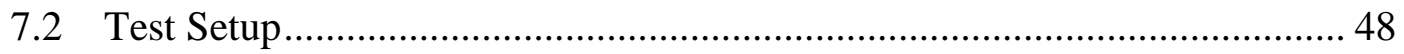

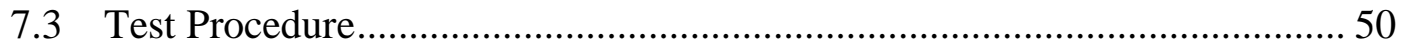

CHAPTER 8 PROCESSING THE HYDROPHONE TEST RESULTS.................... 53

8.1 Free Field Voltage Sensitivity....................................................................... 53 


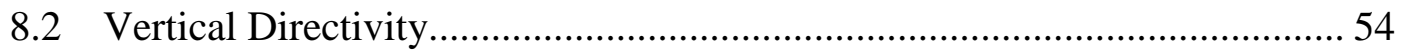

8.3 Horizontal Directivity ............................................................................... 56

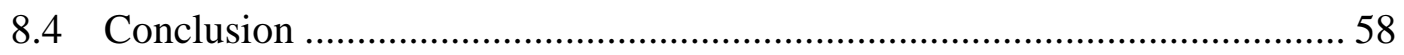

CHAPTER 9 ARRAY FABRICATION …………….......................................... 59

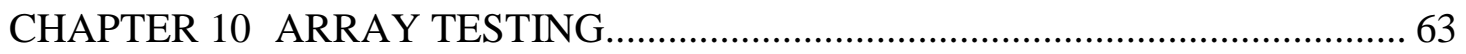

CHAPTER 11 PROCESSING THE ARRAY TEST RESULTS ………………….... 65

11.1 Free Field Voltage Sensitivity........................................................................ 65

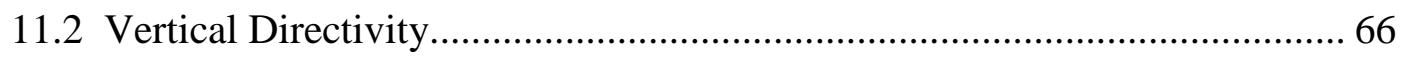

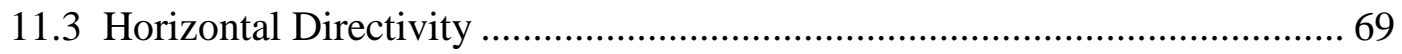

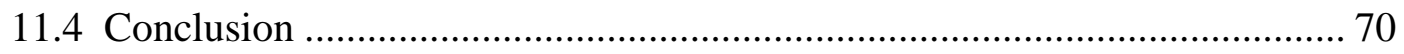

CHAPTER 12 SONAR EQUATION ANALYSIS …………………………......... 71

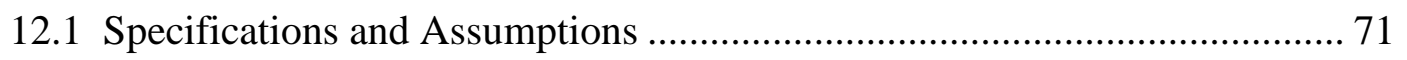

12.2 Performance Analysis of Existing TPL-25 System ....................................... 72

12.3 Performance Analysis of Proposed Design (Multimode Linear Array)........ 76

12.4 Comparison of Proposed Design to Existing System .................................... 78

CHAPTER 13 SUMMARY AND CONCLUSION …………………..................... 79

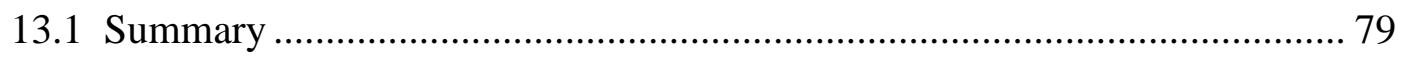

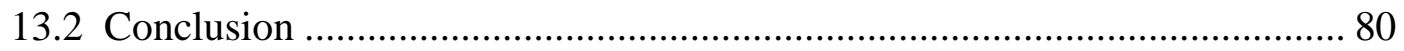

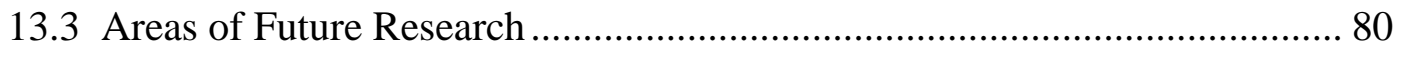

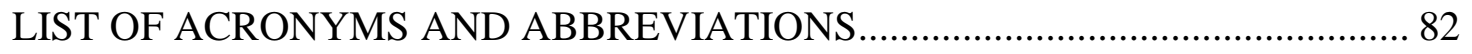

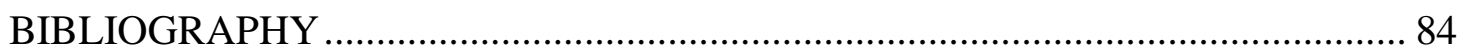




\section{LIST OF FIGURES}

FIGURE

PAGE

Figure 1 A towed pinger locator being used in a search for a black box (Sasse, 2014) 1

Figure 2 The U.S. Navy’s Towed Pinger Locator 25 (NAVSEA).............................. 3

Figure 3 Australian Defence Vessel (ADV) Ocean Shield (Bloomberg, 2014) ........... 5

Figure 4 Chart showing ADV Ocean Shield towed pinger locator detections as of

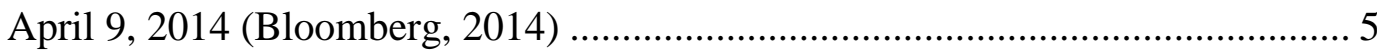

Figure 5 Spherical coordinate system (Acoustical Society of America, 2012)............ 9

Figure 6 Theoretical 2-D multimode beam patterns (Butler, Butler and Rice, 2004) 15

Figure 7 Theoretical multimode 2-D beam patterns ............................................ 17

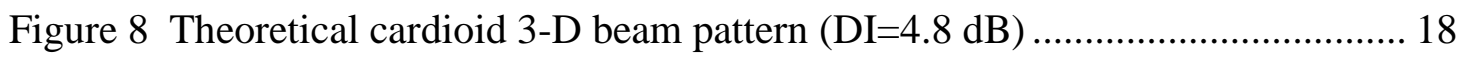

Figure 9 STEMiNC part SMC2622T13111SP (Steiner \& Martins, Inc., 2018)......... 19

Figure 10 PZT cylinders electrode for $3^{\text {rd }}$ and $4^{\text {th }}$ radial extensional modes of vibration

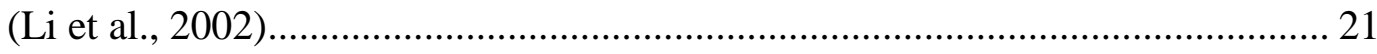

Figure 11 Multimode cylinder transmitting response for the three separate modes of

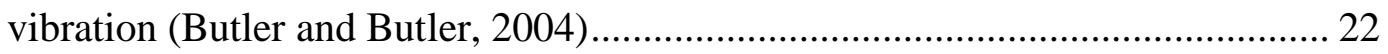

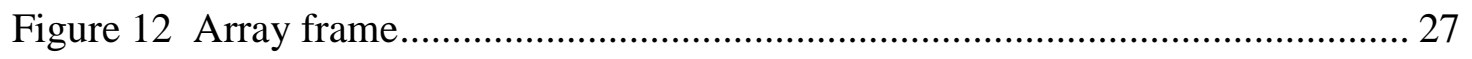

Figure 13 Theoretical horizontal beam patterns for various element spacing ........... 29

Figure 14 Beam patterns for a linear array of 15 point receivers, a single cylinder, and a linear array of 15 cylinders (for $7 / 8$ wavelength spacing) .............................. 30

Figure 15 Cylinder and rubber ring.............................................................. 31

Figure 16 Multimode linear hydrophone array design (all dimensions are in meters)32

Figure 17 Theoretical 3-D beam pattern for multimode cylinder (DI=5.2 dB) ......... 33 
Figure 18 Theoretical 2-D multimode cylinder beam patterns ............................... 34

Figure 19 Theoretical 3-D beam pattern for linear array of 15 isotropic sensors (DI = $14.0 \mathrm{~dB})$. 35

Figure 20 Theoretical 3-D beam pattern for linear array of 15 multimode cylinders

$(\mathrm{DI}=18.35 \mathrm{~dB})$. 36

Figure 21 Theoretical 2-D beam patterns for linear array of 15 multimode cylinders 37

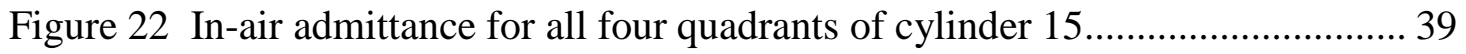

Figure 23 In-air admittance for quadrant 3 of all 15 cylinders ............................... 40

Figure 24 In-air blocked capacitance for all cylinders and segments ..................... 41

Figure 25 In-air leakage resistance for all cylinders and segments ......................... 42

Figure 26 In-air dissipation factor for all cylinders and segments ......................... 43

Figure 27 Fabrication of the single-cylinder hydrophone ..................................... 45

Figure 28 Final single-cylinder hydrophone .................................................... 45

Figure 29 NUWC Open Tank Facility pictorial (Paolero and Évora, 2015) ............. 46

Figure 30 NUWC Open Tank Facility photo .................................................... 47

Figure 31 Hydrophone vertical test fixture ........................................................ 51

Figure 32 Hydrophone horizontal test fixture ........................................................ 52

Figure 33 FFVS magnitude vs. frequency for single-cylinder hydrophone................ 53

Figure 34 Hydrophone voltage magnitudes and phases at each angle in the vertical (xz) plane 54

Figure 35 Hydrophone actual vs. theoretical 2-D beam patterns in the vertical (xz) plane 55 
Figure 36 Hydrophone actual vs. theoretical 2-D beam pattern in the horizontal (xy)

plane.

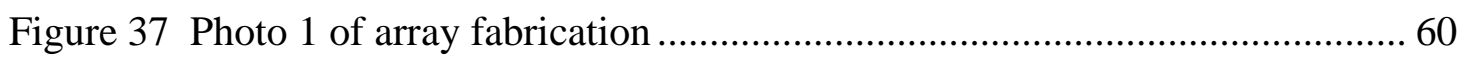

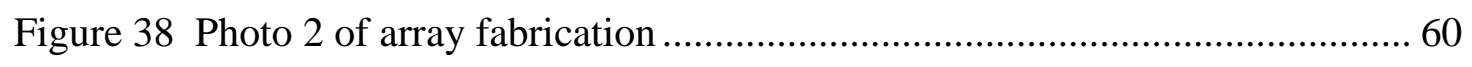

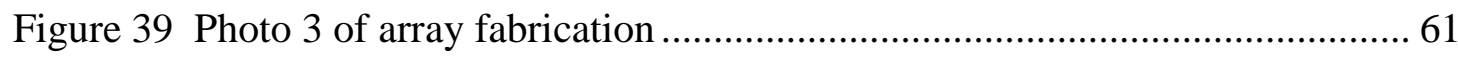

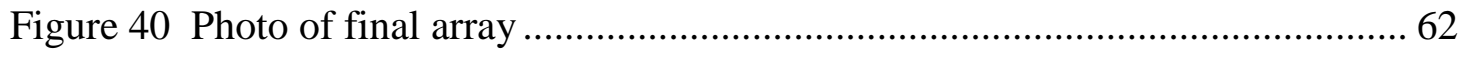

Figure 41 Array Horizontal Test Fixture ........................................................... 64

Figure 42 FFVS magnitude vs. frequency for hydrophone array ............................ 65

Figure 43 Array voltage magnitudes and phases at each angle in the vertical (xz)

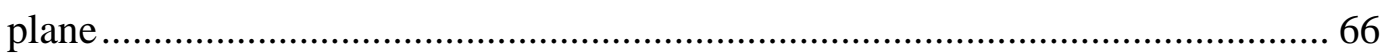

Figure 44 Array actual vs. theoretical 2-D beam patterns in the vertical (xz) plane .. 67

Figure 45 Array actual vs. theoretical 2-D beam pattern in the horizontal (xy) plane 69

Figure 46 Transmission Loss and Figure of Merit of the Existing TPL-25 System ... 75

Figure 47 Transmission Loss and Figure of Merit of the Proposed Design .............. 77 


\section{LIST OF TABLES}

TABLE

PAGE

Table 1 Emergency beacon measurements (BEA, 2011) ....................................... 2

Table 2 Theoretical directivity indexes and beam widths...................................... 18

Table 3 Cylinder material properties................................................................ 19

Table 4 Basic voltage distributions that excite each fundamental mode of vibration 24

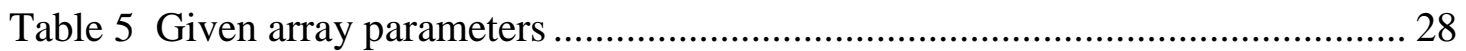

Table 6 Array measurements ........................................................................ 31

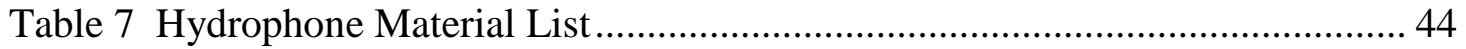

Table 8 Open Tank Facility Test Equipment ..................................................... 48

Table 9 Test Conditions October 14, 2016 .............................................................. 49

Table 10 Hydrophone FFVS Magnitude at $37.5 \mathrm{kHz}$........................................... 53

Table 11 Hydrophone actual vs. theoretical vertical directivity indexes and beam

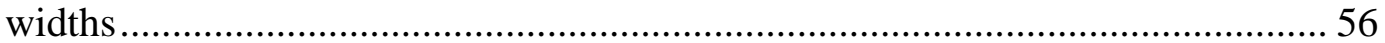

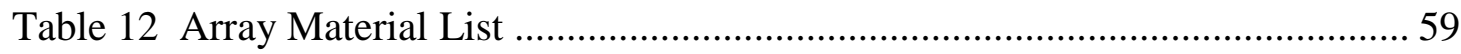

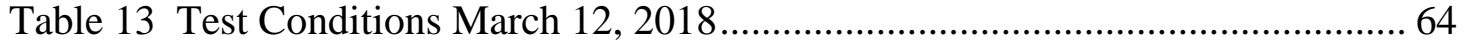

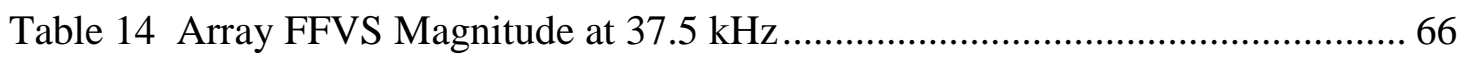

Table 15 Hydrophone actual vs. theoretical vertical directivity indexes and beam

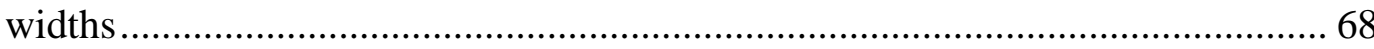

Table 16 Actual vs. theoretical horizontal directivity indexes and beam widths........ 70

Table 17 Summary of existing TPL-25 system performance analysis ...................... 76

Table 18 Summary of proposed design performance analysis.............................. 78

Table 19 Comparison of FOM and detection range ........................................... 78 


\section{CHAPTER 1 INTRODUCTION}

\subsection{Background}

A towed pinger locator is a towed underwater platform used to locate cockpit voice recorders and flight data recorders - commonly referred to as black boxes from downed aircraft. The towed pinger locator's hydrodynamic shell is connected by a cable and winch to a surface vessel, and towed across the search area. An acoustic listening device mounted under the towed pinger locator listens for the acoustic signal (“ping”) being transmitted by an acoustic transmitter that is fitted to the aircraft's black boxes. These acoustic transmitters - commonly referred to as emergency beacons, pingers, or underwater locator beacons (ULBs) - transmit at a frequency of around $37.5 \mathrm{kHz}$ (BEA, 2011). Figure 1 contains a pictorial of a towed pinger locator being used in a search for a flight recorder.

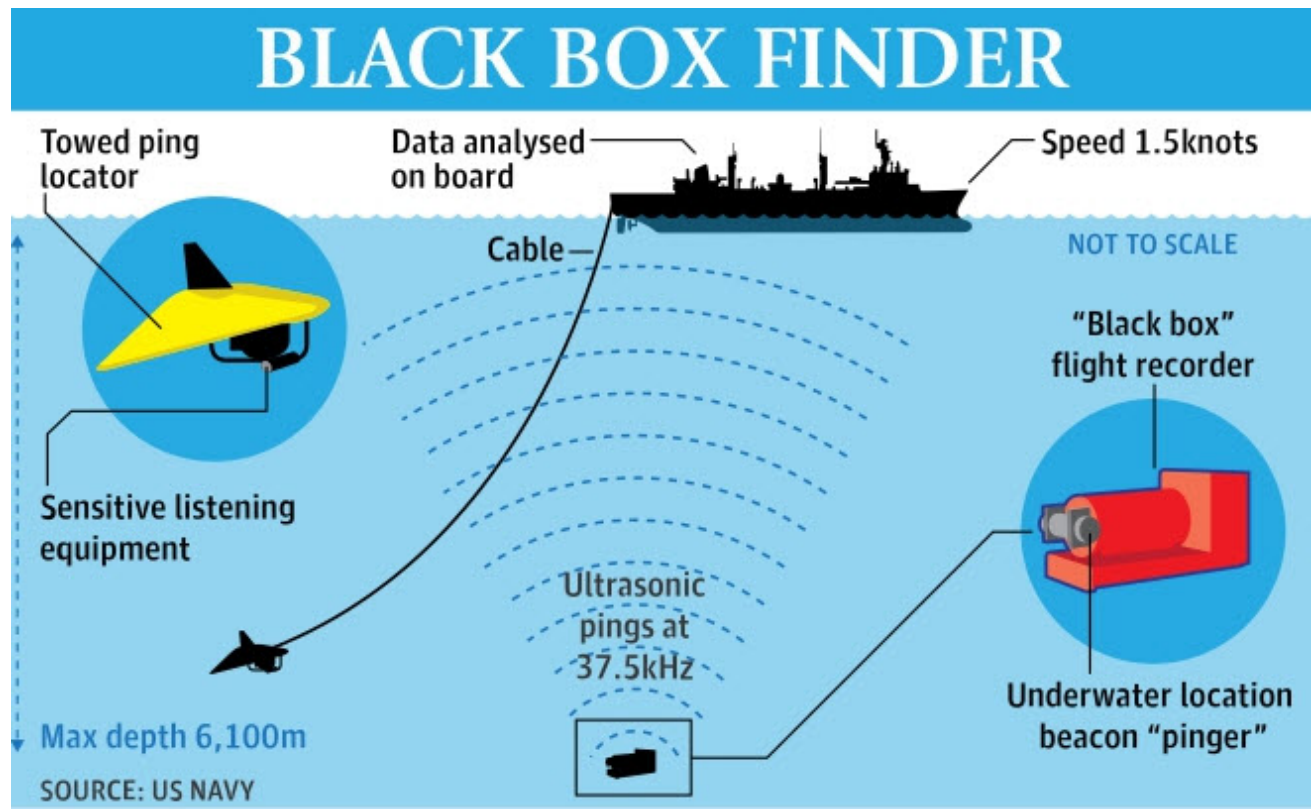

Figure 1 A towed pinger locator being used in a search for a black box (Sasse, 2014) 


\subsubsection{The Emergency Beacon's Transmission Signal}

In August 2011, the Bureau d’Enquêtes et d'Analyses pour la sécurité de l'aviation civile (translated as "Bureau of Enquiry and Analysis for civil aviation safety") called for the measurement of aircraft emergency beacons. Six Dukane DK 120 and six Benthos ELP 362 D emergency beacons were included in this test. The beacons and the hydrophones were immersed to $10 \mathrm{~m}$ with a separation distance of $3.75 \mathrm{~m}$. Table 1 shows the results of the measurement, and is included to provide additional details regarding the beacon's transmission signal.

Table 1 Emergency beacon measurements (BEA, 2011)

\begin{tabular}{ccccccc}
\hline \hline Make & Model & Reference & Level & Recurrence & Duration & Signal $^{1}$ \\
\hline Dukane & DK 120 & SC67204 & $162.7 \mathrm{~dB}$ & 1.069 sec. & $9.38 \mathrm{~ms}$ & FP \\
Dukane & DK 120 & SC72955 & $162.8 \mathrm{~dB}$ & $1.067 \mathrm{sec}$. & $9.34 \mathrm{~ms}$ & FP \\
Dukane & DK 120 & ST17247 & $159 \mathrm{~dB}$ & $1.080 \mathrm{sec}$. & $9.23 \mathrm{~ms}$ & FP \\
Dukane & DK 120 & ST30769 & $162.2 \mathrm{~dB}$ & 1.063 sec. & $9.31 \mathrm{~ms}$ & FP \\
Dukane & DK 120 & ST14872 & $162.5 \mathrm{~dB}$ & 1.085 sec. & $9.18 \mathrm{~ms}$ & FP \\
Dukane & DK 120 & SC31516 & $163 \mathrm{~dB}$ & 1.088 sec. & $9.27 \mathrm{~ms}$ & FP \\
Benthos & ELP 362 D & 47975 & $160.5 \mathrm{~dB}$ & 1.011 sec. & $11.69 \mathrm{~ms}$ & FM \\
Benthos & ELP 362 D & 35651 & $160.8 \mathrm{~dB}$ & 1.000 sec. & $11.70 \mathrm{~ms}$ & FM \\
Benthos & ELP 362 D & 40125 & $160 \mathrm{~dB}$ & $1.030 \mathrm{sec}$. & $12.88 \mathrm{~ms}$ & FM \\
Benthos & ELP 362 D & 46451 & $169.5 \mathrm{~dB}$ & 1.138 sec. & $15.26 \mathrm{~ms}$ & FM \\
Benthos & ELP 362 D & 45401 & $158.6 \mathrm{~dB}$ & $1.021 \mathrm{sec}$. & $12.65 \mathrm{~ms}$ & FM \\
Benthos & ELP 362 D & 48113 & $161.3 \mathrm{~dB}$ & $1.010 \mathrm{sec}$. & $11.93 \mathrm{~ms}$ & FM \\
\hline \hline
\end{tabular}

${ }^{1}$ The DK 120 beacon transmission signal is a pure frequency (FP) between 35.4 and $35.6 \mathrm{kHz}$. The ELP $362 \mathrm{D}$ beacon transmission signal is a frequency modulation (FM descending) from 37.6 to $37.2 \mathrm{kHz}$. 


\subsubsection{The Towed Pinger Locator 25}

The U.S Navy’s system for locating black boxes from downed aircraft is the Towed Pinger Locator 25 (TPL-25). The TPL-25, which was first fielded in 2010, is shown in Figure 2. It is the Navy's fourth generation towed pinger locator - the first was developed in 1985 and was used on numerous civilian and military search operations including Air France flight 447 (Sasse, 2014). The TPL-25 is one of several ocean search and recovery systems maintained and utilized by the Naval Sea Systems Command's (NAVSEA) Supervisor of Salvage and Diving (SUPSALV).

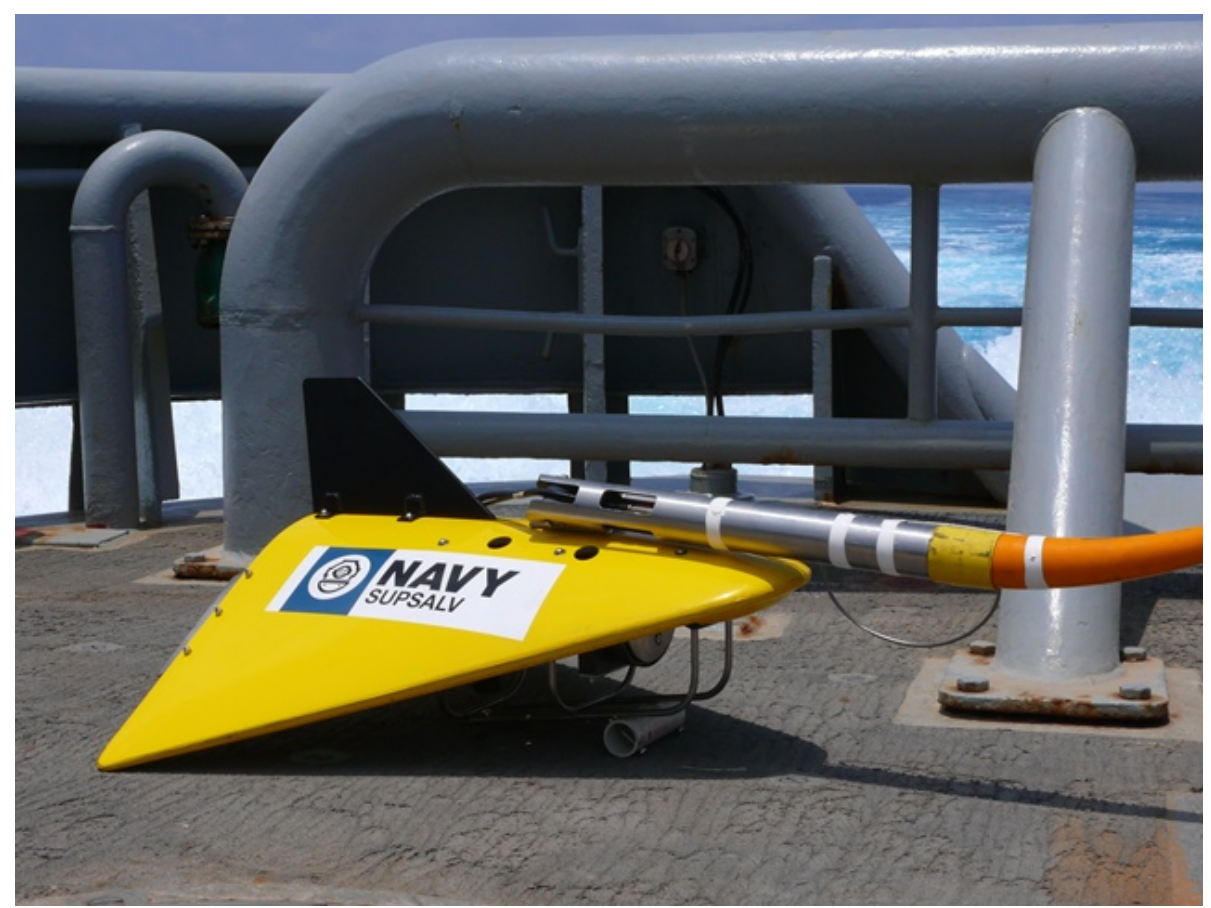

Figure 2 The U.S. Navy’s Towed Pinger Locator 25 (NAVSEA)

The TPL-25 consists of a tow fish (a 70-lb fiberglass shell, 30 inches in length and 35 inches in diameter ${ }^{2}$ ), tow cable, winch, hydraulic power unit, generator, and topside control console. It is towed behind a vessel at slow speeds, generally from 1

\footnotetext{
2 The specification does not describe how the "diameter" of the tow fish is measured.
} 
to 5 knots, depending on the depth. The TPL-25 contains a single omnidirectional hydrophone (listening device) within a 1-standard atmosphere titanium housing rated for a maximum depth of 6,100 meters (Sasse, 2014). The TPL-25 therefore has a maximum operating depth of 6,100 meters or 20,000 feet.

\subsection{Significance}

Towed pinger locators have been successful at locating numerous downed aircraft in the past, including the Adam Air Flight 574, which crashed on January 1, 2007 while flying over the Makassar Strait, killing all 102 people on board. The U.S. Navy found it at a depth of between 4,900 and 6,200 feet using a towed pinger locator on the USNS Mary Sears (Lamothe, 2014). More recently, on December 29, 2017, a U.S. Navy SUPSALV team using the TPL-25 discovered the wreckage of a C-2A Greyhound aircraft that crashed into the Philippine Sea on November 22, 2017 while en route to the USS Ronald Reagan. The wreckage was discovered at a depth of 18,500 feet (U.S. $7^{\text {th }}$ Fleet Public Affairs, 2018).

However, the TPL-25 was unsuccessful in its search for Malaysia Airlines Flight 370 which disappeared March 8, 2014 with 239 people on a route from Kuala Lumpur in Malaysia to Beijing, China. Figure 3 shows a photo of the Australian Defence Vessel (ADV) Ocean Shield towing a TPL-25 in the search for Malaysia Airlines Flight 370. The Ocean Shield believed that it had detected two signals from the aircraft's flight recorder on April 5, 2014 and two more on April 8, 2014 (Figure 4). One thing that may have hindered the detection of Flight 370 by the TPL-25 was the depth of the Indian Ocean where the airline crashed, which was believed to be as deep as 23,000 feet. (Bloomberg, 2014) 


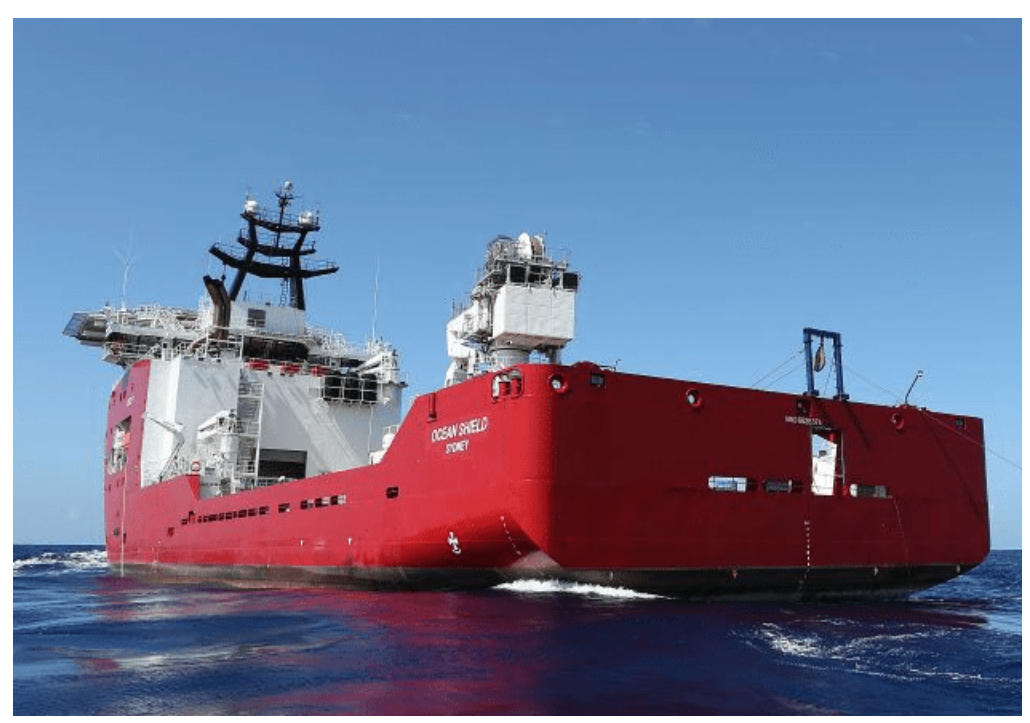

Figure 3 Australian Defence Vessel (ADV) Ocean Shield (Bloomberg, 2014)

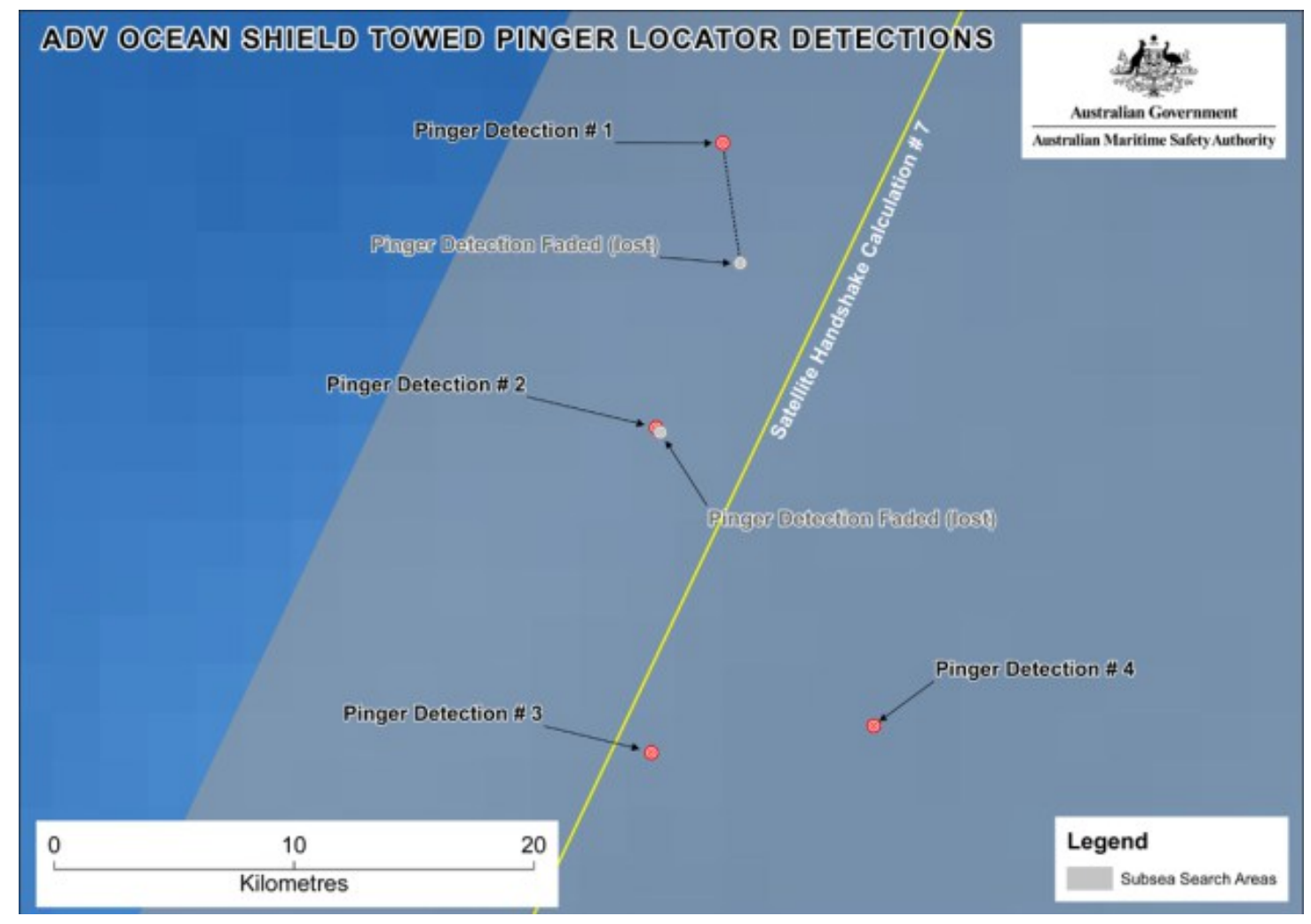

Figure 4 Chart showing ADV Ocean Shield towed pinger locator detections as of April 9, 2014 (Bloomberg, 2014) 
Recovery of a downed aircraft's black boxes is critical to an accident investigation because the information they provide is essential to determining the cause of the accident. However, the window in which to locate the black boxes is short. The typical battery on a black box's emergency beacon begins to fade after 35 days and loses meaningful output after 38 to 40 days (Bloomberg, 2014).

One way to increase the chances of locating the emergency beacons during this window of time is to increase the detection range of the TPL-25. Designing an improved sonar system for the TPL-25, one that that will increase the TPL-25's detection range, is the goal of this thesis.

\subsection{Initial Study}

In 2015, a preliminary analysis of the performance of the existing TPL-25 system and the performance of an upgraded design that incorporated a linear array of 32 omnidirectional hydrophones was conducted (Alvanas, 2015). The preliminary analysis estimated the Transmission Loss (TL), Directivity Index (DI), Detection Threshold (DT), Figure of Merit (FOM) and detection range $(r)$ of the existing and upgraded systems. The analysis concluded that the upgraded design (32-hydrophone linear array) yielded a $40 \%$ increase in detection range, with estimated detection ranges of 2,751 meters for the upgraded design and 1,963 meters for the existing TPL25 system.

\section{$1.4 \quad$ Hypothesis}

Detection range $(r)$ can be predicted through the Transmission Loss (TL) parameter (Urick p. 17, 1983) of the passive sonar equation which is defined by Urick (p. 22, 1983) as: 


$$
\mathrm{SL}-\mathrm{TL}=\mathrm{NL}-\mathrm{DI}+\mathrm{DT}
$$

where
SL is Source Level
TL is Transmission Loss
NL is Noise Level
DI is Directivity Index
DT Detection Threshold

This equation is discussed in more detail later in the thesis. For now, it is only necessary to understand that, based upon this equation, Transmission Loss (TL), and therefore detection range $(r)$, can be increased if Directivity Index $(D I)$ is increased.

The hypothesis of this thesis is that if, as shown in the initial study, replacing the TPL-25's single omnidirectional hydrophone with a linear array of omnidirectional hydrophones can affectedly increase the detection range $(r)$ of the TPL-25, then replacing the single hydrophone with a linear array of directional hydrophones (which has a higher Directivity Index (DI) than a linear array of omnidirectional hydrophones) will increase the detection range $(r)$ even more dramatically.

\subsection{Methodology}

The use of multimode technology as a means to increase the directionality of a transducer was first researched.

This multimode technology was then applied to a single-cylinder hydrophone prototype. The theoretical directivity (beam pattern and directivity index) of the single-cylinder multimode hydrophone prototype was predicted, the prototype was fabricated, the prototype was tested in an acoustic test tank, the data collected in the tank was processed using MATLAB, and the actual directivity was compared to the predicted directivity. 
The multimode technology was then applied to a multi-cylinder linear hydrophone array. The array was designed using SOLIDWORKS. And again, the theoretical directivity (beam pattern and directivity index) of the linear hydrophone array was predicted, the array was fabricated, the array was tested in an acoustic test tank, the data collected in the tank was processed using MATLAB, and the actual directivity was compared to the predicted directivity.

Finally, detection ranges were estimated for the existing TPL-25 system and for the TPL-25 with the proposed multi-cylinder multimode linear hydrophone array.

\subsection{Thesis Outline}

The thesis contains thirteen chapters. Chapter 2 provides an overview of the researched technology. Chapter 3 provides the array design. Chapter 4 provides the predicted directivity of both the single-cylinder multimode hydrophone and the multicylinder multimode linear hydrophone array. Chapter 5 provides in-air characterization of the individual cylindrical transducers. Chapter 6 describes the single-cylinder hydrophone prototype fabrication. Chapter 7 describes the hydrophone prototype tank test. Chapter 8 analyzes the data from the hydrophone prototype tank test. Chapter 9 describes the array fabrication. Chapter 10 describes the array tank test. Chapter 11 analyzes the data from the array tank test. Chapter 12 introduces the passive sonar equation and calculates estimated detection range for both the existing TPL-25 system and the TPL-25 with the proposed multi-cylinder multimode linear hydrophone array. Chapter 13 provides a summary and conclusion, and recommends future research areas. 


\section{CHAPTER 2 TECHNOLOGY OVERVIEW}

This chapter includes an overview of the technology that was researched and applied in the following chapters. A number of the terms introduced in this chapter are applicable to both projectors (transmitting transducers) and hydrophones (receiving transducers), however, they are defined here, and used later, primarily in the context of hydrophones.

\subsection{Spherical Coordinate System}

The spherical coordinate system shown in Figure 5 is the coordinate system that is used throughout this thesis to describe the direction of arrival of a sound wave relative to an acoustic aperture. The acoustic aperture is positioned in the coordinate system so that its acoustic center is located at point $\mathrm{x}=\mathrm{y}=\mathrm{z}=0$ and its length extends along the y-axis. The vector $r$ points to the sound wave's direction of arrival. The angle phi, $\emptyset$, is considered the azimuthal angle, and the angle theta, $\theta$, is considered the elevation angle.

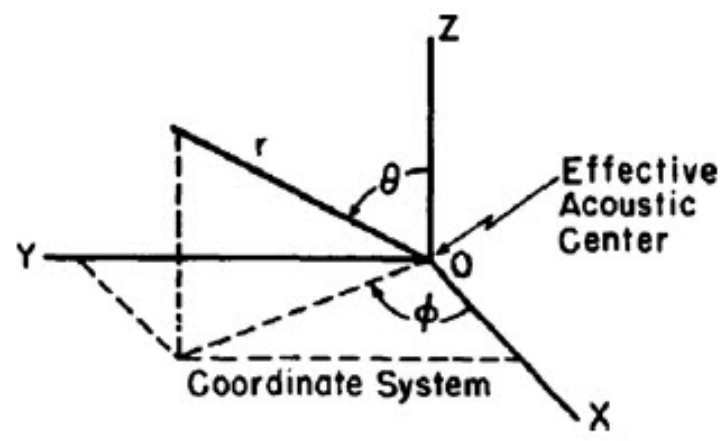

Figure 5 Spherical coordinate system (Acoustical Society of America, 2012)

\subsection{Directionality}

Directionality of an acoustic aperture refers to its ability to focus in a particular direction. Directionality increases sensitivity in the direction of interest, and reduces 
the noise arriving from directions that are not of interest, therefore increasing the overall signal-to-noise ratio (SNR). There are a number of ways to quantify directionality including beam pattern, directivity index and beam width - these terms are introduced in the following sections.

\subsubsection{Beam Pattern}

Beam pattern describes the response of an acoustic aperture as a function of direction. For hydrophones, beam pattern is a function of phi and theta, and is the mean-square voltage produced by an array of unit response when sound of unit pressure is incident on it in the direction of phi, theta (Urick p. 54, 1983). Beam pattern is the voltage response at each arrival angle, normalized so that its maximum value is 1 , and then squared.

At times within this text, there are references to three-dimensional (3-D) beam patterns, and at other times there are references to two-dimensional (2-D) beam patterns. A 3-D beam pattern is a function of both azimuthal angle phi and elevation angle theta and is referred to as $b(\emptyset, \theta)$. A 2-D beam pattern can exist in either the xy plane, where it is a function of the azimuthal angle phi and is referred to as $b(\varnothing)$, or it can exist in the xz plane, where it is a function of the elevation angel theta and is referred to as $b(\theta)$.

The 2-D beam pattern at elevation angle $\theta$ is simply the 3-D beam pattern averaged over all azimuthal angles (Ellis, 2006):

$$
b(\theta)=\frac{1}{2 \pi} \int_{0}^{2 \pi} b(\varnothing, \theta) d \emptyset
$$


Likewise, the 2-D beam pattern at azimuthal angle $\emptyset$ is simply the 3-D beam pattern averaged over all elevation angles (Ellis, 2006):

$$
b(\varnothing)=\frac{1}{2 \pi} \int_{0}^{2 \pi} b(\varnothing, \theta) d \theta
$$

One of the beam patterns discussed in this thesis is the beam pattern for a continuous linear aperture of length $L$ and wavelength $\lambda$. The 2-D beam pattern is given by Urick (p. 43, 1983) as:

$$
\mathrm{b}(\emptyset)=\left[\frac{\sin (\pi \mathrm{L} / \lambda) \sin \emptyset}{(\pi \mathrm{L} / \lambda) \sin \emptyset}\right]^{2}
$$

The 3-D beam pattern is:

$$
\mathrm{b}(\varnothing, \theta)=\left[\frac{\sin (\pi \mathrm{L} / \lambda) \sin \emptyset}{(\pi \mathrm{L} / \lambda) \sin \emptyset} \cdot \sin \theta\right]^{2}
$$

Another beam pattern that is addressed in this thesis is the beam pattern for a linear array of $N$ equally spaced elements. This beam pattern is a derivation of the array factor, which is the spatial Fourier transform of the element excitations and is defined by Balanis (2005) as:

$$
A F(\varnothing)=\sum_{n=0}^{N-1} a_{n} e^{j k d \cos \emptyset}
$$

where

$a_{n}$ is the excitation coefficient of each element

$k$ is the wavenumber, $2 \pi / \lambda$

$\lambda$ is the acoustic wavelength

$d \quad$ is the distance between elements

For uniform element excitation (the case in this thesis), $a_{n}$ can be set to $1 / \mathrm{N}$ for each element and the array factor becomes: 


$$
\operatorname{AF}(\varnothing)=\frac{1}{N} \sum_{n=0}^{N-1} e^{\mathrm{jnkd} \cos \varnothing}
$$

The 3-D array factor is:

$$
\mathrm{AF}(\varnothing, \theta)=\frac{1}{\mathrm{~N}} \sum_{\mathrm{n}=0}^{\mathrm{N}-1} \mathrm{e}^{\mathrm{jnkd} \cos \emptyset \sin \theta}
$$

The beam pattern is the magnitude squared of the array factor. The 2-D and 3-D beam patterns are defined respectively as:

$$
\begin{gathered}
b(\varnothing)=|A F(\varnothing)|^{2}=\left|\frac{1}{N} \sum_{n=0}^{N-1} e^{j n k d \cos \emptyset}\right|^{2} \\
b(\varnothing, \theta)=|\operatorname{AF}(\varnothing, \theta)|^{2}=\left|\frac{1}{N} \sum_{n=0}^{N-1} e^{j n k d \cos \emptyset \sin \theta}\right|^{2}
\end{gathered}
$$

\subsubsection{Product Theorem}

The product theorem states that the beam pattern of an array of identical, equally spaced directional hydrophones is the product of (1) the pattern of each hydrophone alone, and (2) the pattern of an identical array of nondirectional hydrophones (Urick p. $60,1983)$. In this thesis, the product theorem is used to determine the beam pattern for a linear array of equally spaced cylinders.

The 2-D beam pattern for a linear array of equally spaced cylinders is the product of the beam pattern for a cylinder and the beam pattern for a linear array of $\mathrm{N}$ equally spaced elements:

$$
\mathrm{b}(\varnothing)=\left[\frac{\sin [(\pi \mathrm{L} / \lambda) \sin \varnothing]}{(\pi \mathrm{L} / \lambda) \sin \varnothing}\right]^{2}\left[\frac{1}{\mathrm{~N}} \sum_{\mathrm{n}=0}^{\mathrm{N}-1} \mathrm{e}^{\mathrm{jnkd} \cos \varnothing}\right]^{2}
$$


Likewise, the 3-D beam pattern is the following product:

$$
\mathrm{b}(\emptyset, \theta)=\left[\frac{\sin [(\pi \mathrm{L} / \lambda) \sin \emptyset]}{(\pi \mathrm{L} / \lambda) \sin \varnothing} \cdot \sin \theta\right]^{2}\left[\frac{1}{\mathrm{~N}} \sum_{\mathrm{n}=0}^{\mathrm{N}-1} \mathrm{e}^{\mathrm{jnkd} \cos \emptyset \sin \theta}\right]^{2}
$$

\subsubsection{Array Gain}

Array gain $(A G)$ is the ratio of the signal-to-noise ratio of the array to the signalto-noise ratio of a single element of the array, and is defined in decibels by Urick (p. 34, 1983) as:

$$
\mathrm{AG}=10 \log \frac{(\mathrm{S} / \mathrm{N})_{\text {array }}}{(\mathrm{S} / \mathrm{N})_{\text {one element }}}
$$

When the signal is a unidirectional plane wave and is therefore perfectly coherent, array gain can be reduced to the ratio of the noise power out of one element to the noise power out of the array (Burdic, 1991):

$$
A G=10 \log \frac{\int_{4 \pi}|\mathrm{N}(\varnothing, \theta)|^{2} \mathrm{~d} \Omega}{\int_{4 \pi}|\mathrm{N}(\varnothing, \theta)|^{2} \mathrm{~b}(\varnothing, \theta) \mathrm{d} \Omega}
$$

where

$$
\begin{array}{ll}
\Omega & \text { is a solid angle given by } \varnothing \text { and } \theta \\
b(\emptyset, \theta) & \text { is the beam pattern of the array }
\end{array}
$$

\subsubsection{Directivity Index}

When the noise is isotropic such that $N(\varnothing, \theta)=1$, the array gain of Equation (14) can be further reduced to a quantity called directivity index (Urick p. 42, 1983):

$$
\mathrm{DI}=10 \log \frac{\int_{4 \pi} \mathrm{d} \Omega}{\int_{4 \pi} \mathrm{b}(\varnothing, \theta) \mathrm{d} \Omega}=10 \log \frac{4 \pi}{\int_{0}^{2 \pi} \int_{-\pi / 2}^{\pi / 2} \mathrm{~b}(\varnothing, \theta) \cos \varnothing \mathrm{d} \varnothing \mathrm{d} \theta}
$$

If the beam pattern has rotational symmetry and is nondirectional in elevation, then the Directivity Index (DI) can be reduced to (Urick p. 42, 1983): 


$$
\mathrm{DI}=10 \log \frac{4 \pi}{2 \pi \int_{-\pi / 2}^{\pi / 2} b(\varnothing) \cos \emptyset \mathrm{d} \emptyset}
$$

Likewise, if the beam pattern has rotational symmetry and is nondirectional in azimuth, then the directivity index can be reduced to:

$$
\mathrm{DI}=10 \log \frac{4 \pi}{2 \pi \int_{-\pi / 2}^{\pi / 2} b(\theta) \cos \theta d \theta}
$$

The above directivity indexes are referred to in this text as 3-D directivity indexes because they are measures of a 3-D beam pattern. Often, however, a 3-D beam pattern is not available. If a hydrophone is rotated vertically and directivity measurements are taken, a 2-D vertical beam pattern can be created. If the hydrophone is then rotated horizontally and directivity measurements are taken, a 2-D horizontal beam pattern can be created. In these cases, when there are one or more 2-D beam patterns but not a 3-D beam pattern, only the 2-D directivity indexes can be calculated as follows:

$$
\begin{gathered}
D_{\text {horizontal }}=10 \log \frac{\int_{0}^{2 \pi} d \varnothing}{\int_{0}^{2 \pi} b(\varnothing) d \emptyset}=10 \log \frac{2 \pi}{\int_{0}^{2 \pi} b(\varnothing) d \emptyset} \\
D_{\text {vertical }}=10 \log \frac{\int_{0}^{2 \pi} d \theta}{\int_{0}^{2 \pi} b(\theta) d \theta}=10 \log \frac{2 \pi}{\int_{0}^{2 \pi} b(\theta) d \theta}
\end{gathered}
$$

Directivity index is defined by Nuttall and Cray (2001) as “a decibel measure of the improvement in the SNR that a beamformed array provides in an ideal isotropic noise field with a perfectly correlated signal, relative to an omnidirectional array element in the free field".

\subsubsection{Beam Width}

Beam width is the angle between the points on each side of the main response axis where the sound pressure level is at some specified level relative to the main 
response level. A standard beam width level is $-3 \mathrm{~dB}$, because this is the half power level. The beam width is a measure of the sharpness of the main lobe of the beam pattern.

\subsection{Multimode Transducer Technology}

Multimode transducer technology is a technology that exploits the fundamental vibration modes of a transducer in order to create a particular directional transmit or receive pattern. An overview of this technology as it applies to the cylindrical hydrophone (the subject of this thesis) and the specific goal of increased directivity is provided in the following sections.

\subsubsection{Multimode Beam Patterns}

A multimode beam pattern is created by combining the beam patterns associated with the natural vibration modes of the device shape. The focus of this section (and throughout this paper) is the creation of the multimode cardioid beam pattern through the combination of the first two extensional modes of vibration of the cylinder, the omnidirectional $(n=0)$ mode and the dipole $(n=1)$ mode (Figure 6).

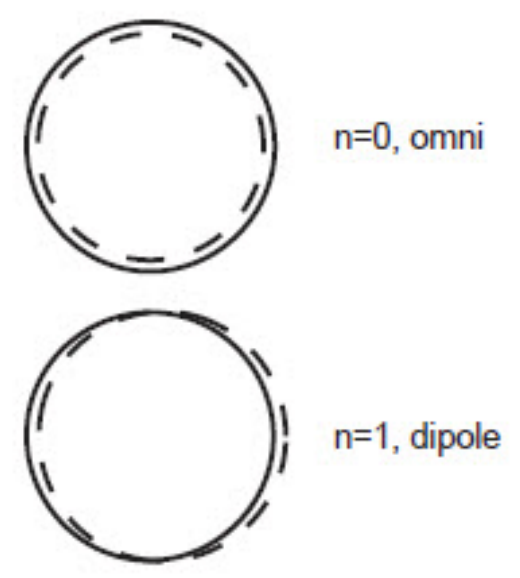

Figure 6 Theoretical 2-D multimode beam patterns (Butler, Butler and Rice, 2004) 


\section{Omni Beam Pattern}

The omni (or omnidirectional) mode is the fundamental extensional "breathing" mode, where the expansion is uniform and in phase at all locations along the circumference of the cylinder. The cylinder vibrates in the radial direction as a single mass as a result of the expansion and contraction of the circumference (Sherman and Butler, 2007). The 2-D omni beam pattern is given by the following equation:

$$
b(\varnothing)=1
$$

and is shown in Figure 7(a). When this beam pattern is plugged into Equation (18), the resulting 2-D directivity index is $0 \mathrm{~dB}$. The beam width is considered to be 360 degrees.

\section{Dipole Beam Pattern}

The dipole mode has a figure eight beam pattern where the two lobes have the same magnitude of expansion but are 180 degrees out of phase, so that when one half of the cylinder is expanding, the other half is contracting. According to Sherman \& Butler (2007), the dipole has a 3-D DI of $4.8 \mathrm{~dB}$, a 90 degree beam width, and a 2-D beam pattern given by the following equation:

$$
b(\varnothing)=\cos \emptyset^{2}
$$

When this beam pattern is plugged into Equation (18), the resulting 2-D directivity index is $3.0 \mathrm{~dB}$. The dipole 2-D beam pattern is shown in Figure 7(b).

\section{Cardioid Beam Pattern}

When the omni and dipole beam patterns are combined with equal weight, a cardioid (heart-shaped) beam pattern is created. ${ }^{3}$ This cardioid is sometimes referred

\footnotetext{
${ }^{3}$ Other weights can be applied to the dipole to generate variations of the cardioid such as the supercardioid (or maximum-DI cardioid). These variations are not considered in this work.
} 
to as the optimum-null cardioid. According to Sherman \& Butler (2007), the cardioid has a 3-D DI of $4.8 \mathrm{~dB}$ (same as the dipole), a 131 degree beam width, and a 2-D beam pattern given by the following equation:

$$
\mathrm{b}(\varnothing)=\left[\frac{1+\cos \emptyset}{2}\right]^{2}
$$

When this beam pattern is plugged into Equation (18), the resulting 2-D directivity index is $4.3 \mathrm{~dB}$. The cardioid 2-D beam pattern is shown in Figure 7(c).

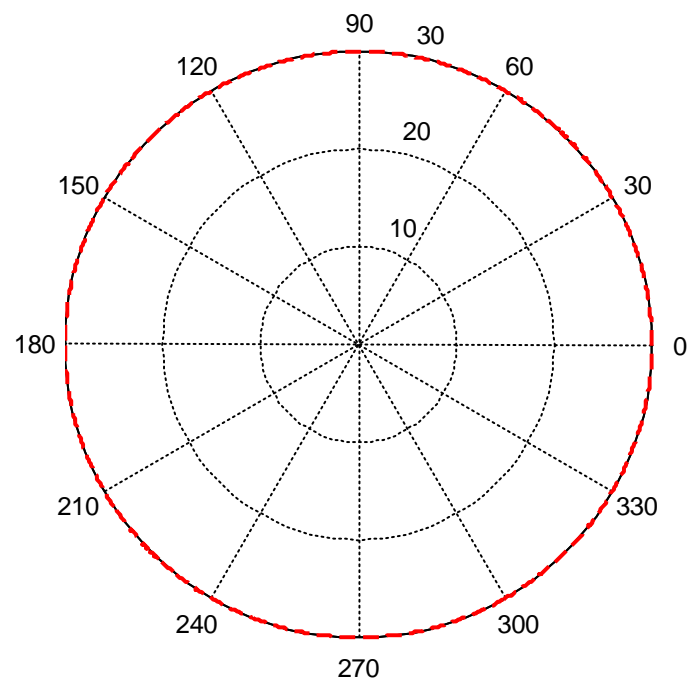

(a) Omni beam pattern

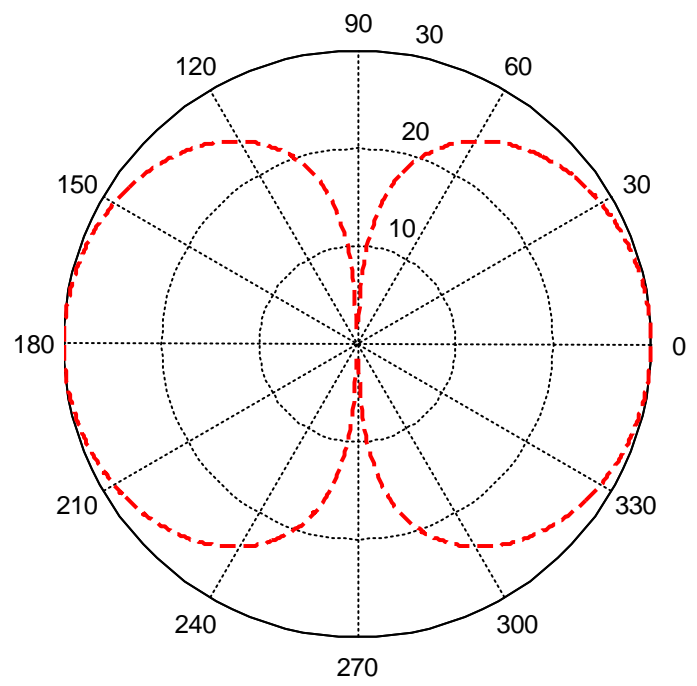

(b) Dipole beam pattern

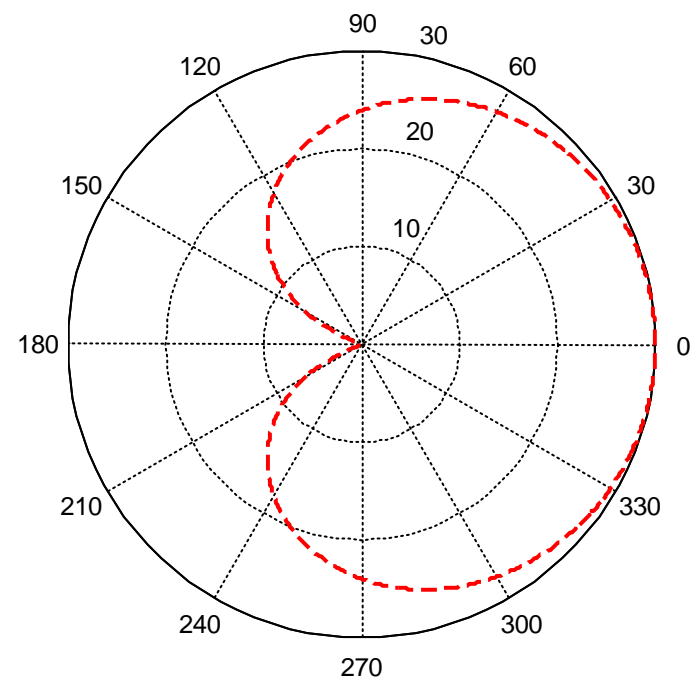

(c) Cardioid beam pattern

Figure 7 Theoretical multimode 2-D beam patterns 
The 3-D cardioid beam pattern is given by McConnell et al. (2006) as:

$$
\mathrm{b}(\varnothing, \theta)=\left[\frac{1+\cos \emptyset \sin \theta}{2}\right]^{2}
$$

The cardioid 3-D beam pattern is shown in Figure 8. This is the 3-D beam pattern for a true cardioid sensor.
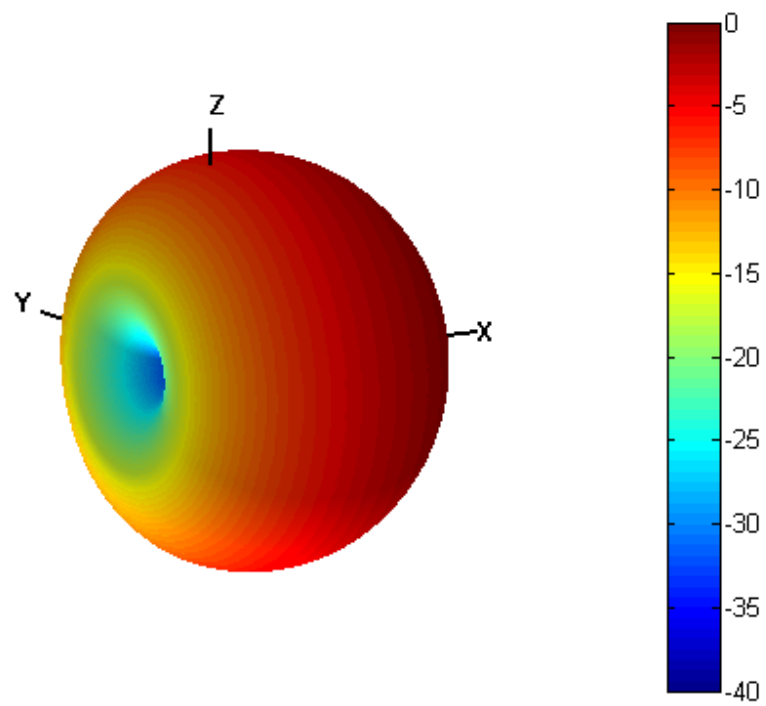

Figure 8 Theoretical cardioid 3-D beam pattern (DI=4.8 dB)

Table 2 contains a summary of the theoretical omni, dipole and cardioid beam pattern characteristic.

Table 2 Theoretical directivity indexes and beam widths

\begin{tabular}{lccc}
\hline \hline Pattern & 3-D DI & 2-D DI & Beam Width \\
\hline Omni & $0.0 \mathrm{~dB}$ & $0.0 \mathrm{~dB}$ & $360 \mathrm{deg}$. \\
Dipole & $4.8 \mathrm{~dB}$ & $3.0 \mathrm{~dB}$ & $90.0 \mathrm{deg}$. \\
Cardioid & $4.8 \mathrm{~dB}$ & $4.3 \mathrm{~dB}$ & $131.8 \mathrm{deg}$. \\
\hline \hline
\end{tabular}




\subsubsection{Multimode Cylinders}

The type of multimode transducer that is the focus of this thesis is the 4-quadrant striped cylinder. The particular cylinder is manufactured by Steiner \& Martins, Inc. (STEMiNC). It has an outside diameter of $26 \mathrm{~mm}$, inside diameter of $22 \mathrm{~mm}$ and length of $13 \mathrm{~mm}$. It is radially poled, and has a single electrode on the outside, and four electrodes (also referred to as quadrants) on the inside. When the cylinder is wired, each quadrant produces a separate voltage output that is proportional to that quadrant's deformation. The cylinder is shown in Figure 9.

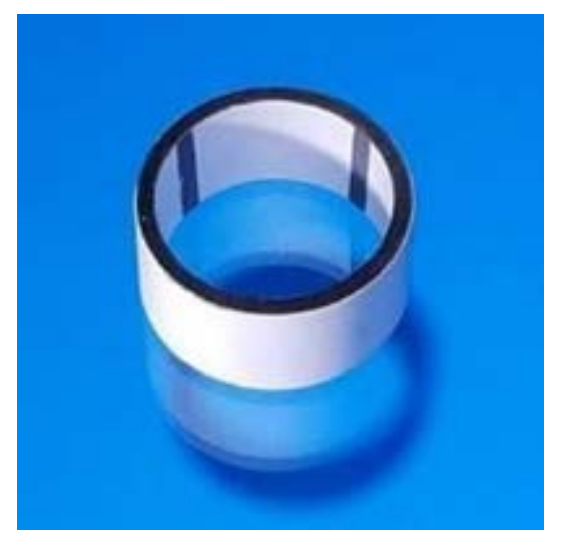

Figure 9 STEMiNC part SMC2622T13111SP (Steiner \& Martins, Inc., 2018)

The cylinder is made of lead zirconate titanate (PZT), and is specified by Steiner \& Martins, Inc. (2018) to be a modified Navy Type I or PZT-4 with the material properties listed in Table 3.

Table 3 Cylinder material properties

\begin{tabular}{|c|c|c|c|}
\hline Property & Units & Symbol & $\begin{array}{c}\text { Value } \\
\text { (modified PZT-4) }\end{array}$ \\
\hline \multirow{3}{*}{$\begin{array}{c}\text { Electromechanical } \\
\text { Coupling } \\
\text { Coefficients }\end{array}$} & & $K_{p}$ & 0.58 \\
\hline & & $K_{t}$ & 0.45 \\
\hline & & $K_{31}$ & 0.34 \\
\hline
\end{tabular}




\begin{tabular}{cccc}
\hline Frequency & $\mathrm{Hz} \cdot \mathrm{m}$ & $N_{p}$ & 2200 \\
Constants & & $N_{t}$ & 2070 \\
& & $N_{31}$ & 1680 \\
\hline Piezoelectric & $\times 10^{-12} \mathrm{~m} / v$ & $D_{33}$ & 320 \\
Constants & & $D_{31}$ & -140 \\
& $\times 10^{-3} \mathrm{Vm} / N$ & $G_{33}$ & 25 \\
& & $G_{31}$ & -11.0 \\
\hline Elastic & $\times 10^{10} \mathrm{~N} / \mathrm{m}^{2}$ & $Y_{33}$ & 7.3 \\
Constants & & $Y_{11}$ & 8.6 \\
\hline Mechanical & & $Q_{m}$ & 1800 \\
Quality Factor & & $\varepsilon T_{33} / \varepsilon_{0}$ & 1400 \\
\hline Dielectric & $@ 1 \mathrm{Khz}$ & $\tan \delta$ & 0.4 \\
Constant & & $T_{c}$ & 320 \\
\hline Dissipation Factor & $\% @ 1 \mathrm{Khz}$ & \\
\hline Curie & ${ }^{\circ} \mathrm{C}$ & $\rho$ & 7.9 \\
Temperature & & $\mathrm{g} / \mathrm{cm}^{3}$ & \\
\hline Density & & \\
\hline \hline
\end{tabular}

This cylinder has a fundamental resonance frequency of $42 \mathrm{kHz}$. This is the resonance frequency of the $\mathrm{n}=0$ or omni mode of vibration, and is defined by Sherman \& Butler (2007) as:

$$
\mathrm{f}_{0}=\frac{\mathrm{c}}{\pi \mathrm{D}}
$$

where

$C$ is the sound speed in the cylinder

$D$ is the mean diameter of the cylinder

For the next mode of vibration, the $\mathrm{n}=1$ or dipole mode, the cylinder's resonance frequency is defined by Sherman \& Butler (2007) as:

$$
\mathrm{f}_{1}=\sqrt{2} \mathrm{f}_{0}
$$

For this cylinder, the dipole mode resonance frequency is $59.4 \mathrm{kHz}$. 
Dividing the inside electrode of a typical piezoelectric cylinder into four (4) or more separate electrodes results in two notable changes in behavior.

The first notable change is the increased prominence of higher modes of vibration in the cylinder. This phenomenon was described in a paper by Hing Leung Li, Jun Hui Hu and Helen Lai Wah Chan (2002) titled "Finite Element Analysis on Piezoelectric Ring Transformer". In it, through both finite element method (FEM) and experimental measurements, they showed that creating multiple concentric electrodes along the thickness of a PZT ring increased the relative level of the higher extensional modes of vibration. The study shows that a ring with a single electrode responds the most to the frequency of its $1^{\text {st }}$ and $2^{\text {nd }}$ radial extensional modes of vibration, a ring with two concentric electrodes responds the most to the frequency of its $3^{\text {rd }}$ radial extensional mode of vibration, and a ring with three concentric electrodes responds most to the frequency of its $4^{\text {th }}$ radial extensional mode of vibration. Figure 10 shows rings with 3 and 2 concentric electrodes respectively. This behavior is explained as a decoupling of the compressive and tensile stress regions by splitting the electrode according to the stress distribution.

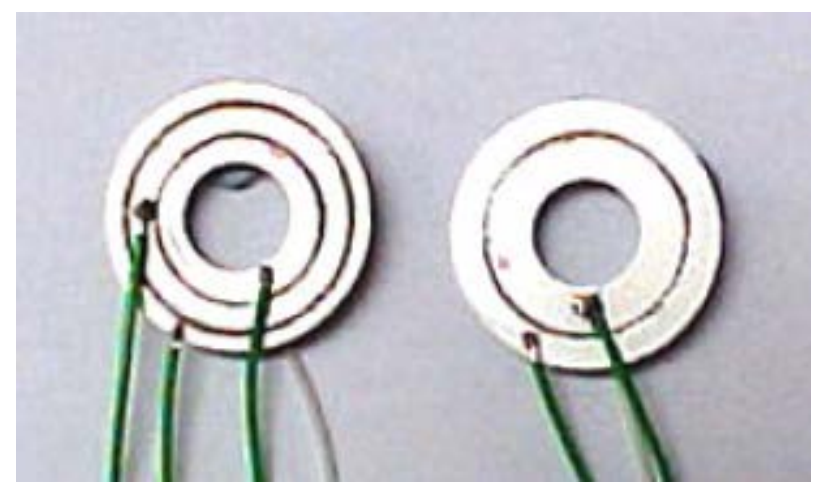

Figure 10 PZT cylinders electrode for $3^{\text {rd }}$ and $4^{\text {th }}$ radial extensional modes of vibration (Li et al., 2002) 
The nodal points for a particular mode of vibration are the locations where the displacement is always zero. On adjacent sides of a nodal point, the forces are in opposite directions. A single electrode resists these opposite forces. But splitting the electrode at the nodal points reduces the resistance and allows for increased elongation in one electrode and increased compression in the next.

Butler and Butler (2004), in their U.S. Patent titled "Multimode synthesized beam transduction apparatus”, describe a PZT cylinder with eight separate electrodes spaced around the inside of the cylinder. They measure the response of the first 3 extensional modes of vibration. This response is shown in Figure 11. This further illustrates how when operating a multimode cylinder at its fundamental breathing resonance frequency, there also exist components of the higher extensional modes of vibration.

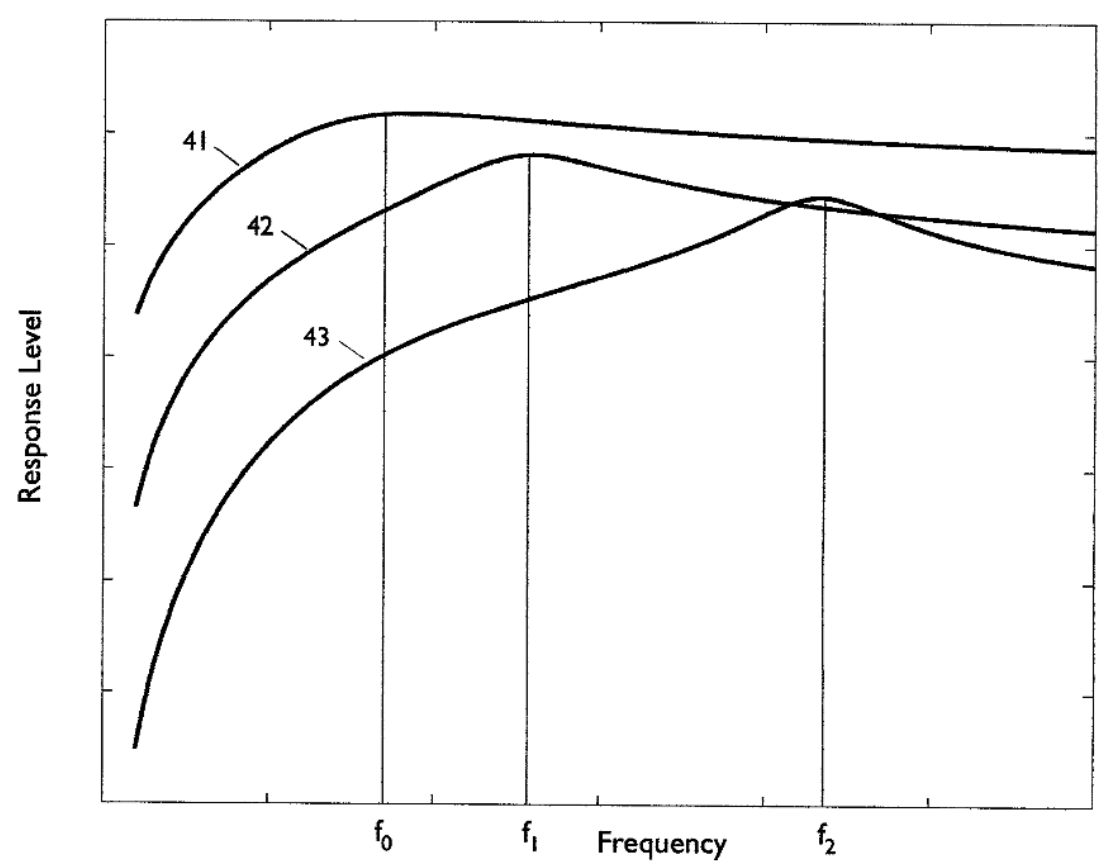

Figure 11 Multimode cylinder transmitting response for the three separate modes of vibration (Butler and Butler, 2004) 
The second notable change when the inside electrode of a typical piezoelectric cylinder is divided into four (4) or more separate electrodes is that the cylinder now functions as a combination of a pressure hydrophone and a pressure gradient hydrophone. The magnitude of the multimode cylinder's omni mode response provides a measure of pressure. The multimode cylinder's dipole mode of vibration is a response to a difference in pressure between one half of the cylinder and the other half, and the magnitude of this dipole mode response is proportional to the pressure gradient.

\subsubsection{Multimode Signal Processing}

The paper titled “A tri-modal directional transducer” by Butler, Butler and Rice (2001) contains a detailed description on how to use multimode cylinders to transmit cardioid beam patterns. Another paper titled "A low-frequency directional flextensional transducer and line array” by Butler, Butler, Butler and Cavanagh (1997) contains additional details on how to transmit cardioid beam patterns.

When a multimode cylinder is used as a projector, an actual cardioid shape beam pattern is transmitted. This is done by applying a specific complex voltage to each electrode so that the resulting vibration has a cardioid shape - no displacement at the point on the cylinder that corresponds to the null of the cardioid, and maximum displacement at the opposite side of the cylinder that corresponds to the cardioid's main response axis. The applied complex voltages are determined by combining the voltages required to generate an omni beam pattern and the voltages required to generate a dipole beam pattern. 
In contrast, when a multimode cylinder is used as a hydrophone, the cylinder does not physically vibrate in this cardioid shape. Instead, the voltages generated at each electrode (the output voltages) are processed in a manner that produces a cardioid receive pattern.

For a multimode cylinder with eight (8) inner electrodes connected in pairs, Butler, Butler and Rice (2004) define the basic voltage distribution that is required to excite the omni and dipole modes of vibration. This voltage distribution is shown in Table 4 , where $V_{1}, V_{2}, V_{3}$ and $V_{4}$ are the complex voltages applied to electrode pairs 1 , 2, 3 and 4, respectively.

Table 4 Basic voltage distributions that excite each fundamental mode of vibration

\begin{tabular}{ccccc}
\hline Mode & $\boldsymbol{V}_{\mathbf{1}}$ & $\boldsymbol{V}_{\mathbf{2}}$ & $\boldsymbol{V}_{\mathbf{3}}$ & $\boldsymbol{V}_{\mathbf{4}}$ \\
\hline Omni & 1 & 1 & 1 & 1 \\
Dipole & 1 & 1 & -1 & -1 \\
\hline \hline
\end{tabular}

This translates into the following equations for a 4-quadrant multimode hydrophone: ${ }^{4}$

$$
\begin{gathered}
\mathrm{V}_{\mathrm{O}}=\mathrm{V}_{1}+\mathrm{V}_{2}+\mathrm{V}_{3}+\mathrm{V}_{4} \\
\mathrm{~V}_{\mathrm{D}}=\mathrm{V}_{1}+\mathrm{V}_{2}-\left(\mathrm{V}_{3}+\mathrm{V}_{4}\right)
\end{gathered}
$$

where

$V_{1}, V_{2}, V_{3}$ and $V_{4}$ are the complex voltages (magnitude and phase) of the voltages measured at quadrants 1, 2, 3 and 4, respectively.

$V_{O}$ is the omnidirectional complex voltage - the sum of the 4 quadrants' complex voltages.

\footnotetext{
${ }^{4}$ Equation (27) is for a maximum response between quadrants 1 and 2.
} 
$V_{D}$ is the dipole complex voltage - the difference between a) the sum of quadrant 1 and 2 complex voltages and $b$ ) the sum of quadrant 3 and 4 complex voltages.

Butler, Butler and Cavanagh (1997) explain that when transmitting a cardioid beam pattern, the applied dipole voltage must be adjusted so that the resulting on-axis pressure amplitude and phase of the dipole beam pattern match that of the omni beam pattern. This creates a dipole pattern that has one lobe with the same pressure magnitude and phase as the omni pattern, and one lobe with the same pressure magnitude but the opposite phase of the omni pattern, so that the one lobe adds to the omni pattern and the other lobe cancels, yielding a null at one side and a pressure increase on the other side. Although the equations used to accomplish this do not translate directly to a multimode cylindrical hydrophone, the general premise was used to arrive at the following equation for a four quadrant multimode hydrophone:

$$
\mathrm{V}_{\mathrm{C}}=\mathrm{V}_{\mathrm{O}}+\mathrm{R} \cdot \mathrm{V}_{\mathrm{D}}
$$

where

$V_{C}$ is the cardioid complex voltage

$R \quad$ is the complex ratio of omni and dipole voltages

$R$ is defined so that the cardioid receive pattern has a null at 180 degrees. In other words, $R$ is defined so that the following condition is met:

$$
\mathrm{V}_{\mathrm{C}}(\pi)=0
$$

Combining Equations 28 and 29 gives:

$$
\mathrm{R} \cdot \mathrm{V}_{\mathrm{D}}(\pi)=-\mathrm{V}_{\mathrm{O}}(\pi)
$$

This results in the following definition for $R$ :

$$
R=\frac{-V_{O}(\pi)}{V_{D}(\pi)}
$$


The magnitudes squared of these complex voltages at each angle create the omni, dipole and cardioid beam patterns as defined respectively below:

$$
\begin{aligned}
& b_{O}(\theta)=\left|V_{O}(\theta)\right|^{2} \\
& b_{D}(\theta)=\left|V_{D}(\theta)\right|^{2} \\
& b_{C}(\theta)=\left|V_{C}(\theta)\right|^{2}
\end{aligned}
$$

These equations are used later in the thesis to process the data that is collected during the tank tests. 


\section{CHAPTER 3 ARRAY DESIGN}

\subsection{Budget Driven Decisions}

A donation of $\$ 1000$ was received to purchase materials for this project. The most significant design decision - how many elements to include in the linear array was based entirely on this budget. There was enough money to purchase 16 cylinders. One of these 16 was allocated to the single-cylinder hydrophone prototype that would be built and tested first. Therefore, the array would include 15 cylinders.

The second design decision that was influenced by budget was how to assemble the cylinders into a linear array. While soliciting design ideas from colleagues, one colleague located an obsolete hydrophone frame that was the perfect size. It comprised six stainless steel rods, 24 inches in length and 1/8 inch in diameter, arranged in parallel, and held in place by 2 cylindrical brass endcaps. The interior space created by the arrangement of the rods was just large enough for the cylindrical transducers to fit into. This frame (shown in Figure 12) was donated to the project and became the backbone of the linear array design.

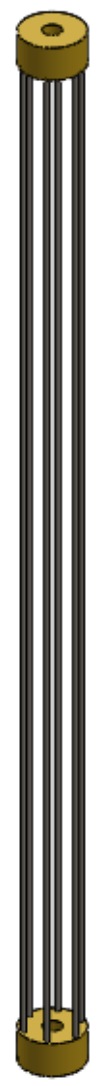

Figure 12 Array frame

\subsection{Element Spacing}

The next design decision was the array element spacing. Given the parameters in Table 5, Equations (11) and (18) were used to calculate the horizontal beam pattern and directivity index for a linear array of 15 cylinders with the distance between 
cylinders, $d$, set to $1 / 2$ wavelength, $3 / 4$ wavelength, $7 / 8$ wavelength and 1 wavelength. The results are shown in Figure 13. A decision was made to proceed with 7/8 wavelength spacing because it generated the highest directivity index, a horizontal DI of $15.8 \mathrm{~dB}$.

Table 5 Given array parameters

\begin{tabular}{ccc}
\hline \hline Parameter & Description & Value \\
\hline $\mathrm{f}$ & Operating Frequency & $37.5 \mathrm{kHz}$ \\
$\mathrm{c}$ & Sound Speed & $1500 \mathrm{~m} / \mathrm{s}$ \\
$\lambda$ & Wavelength $=c / f$ & $4.0 \mathrm{~cm}$ \\
$\mathrm{n}$ & Number of cylinders & 15 \\
$\mathrm{~L}$ & Cylinder Length & $1.3 \mathrm{~cm}$ \\
\hline \hline
\end{tabular}




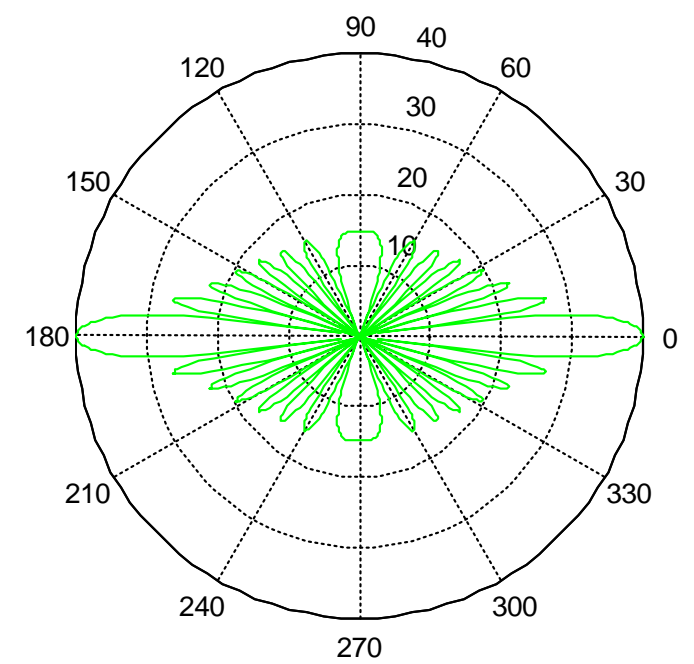

1/2 wavelength spacing (Horizontal DI = $13.66 \mathrm{~dB})$

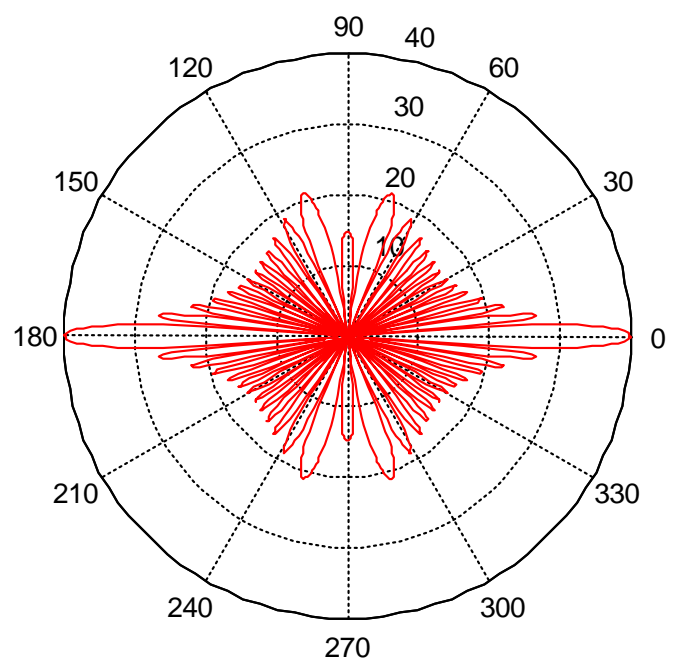

7/8 wavelength spacing (Horizontal DI = $15.77 \mathrm{~dB})$

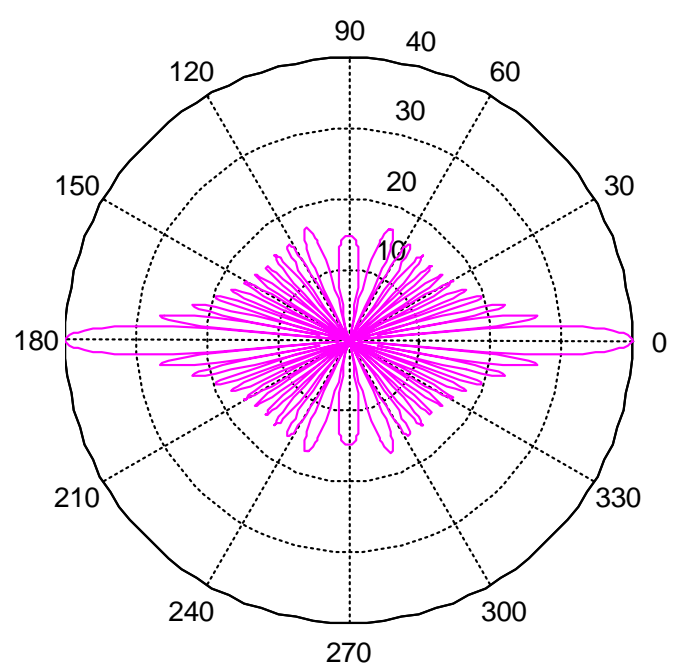

3/4 wavelength spacing (Horizontal $\mathrm{DI}=15.31 \mathrm{~dB}$ )

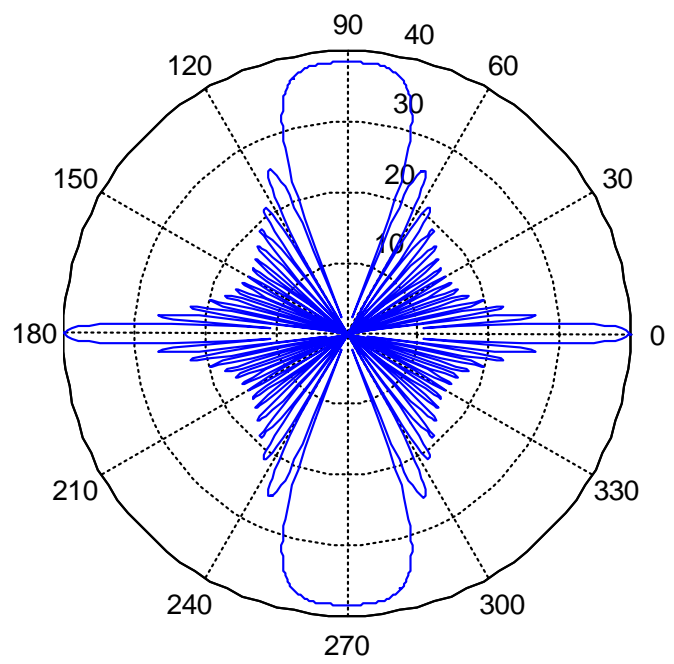

1 wavelength spacing (Horizontal $\mathrm{DI}=8.82 \mathrm{~dB}$ )

Figure 13 Theoretical horizontal beam patterns for various element spacing

According to the product theorem, the beam pattern for a linear array of point receivers was multiplied by the beam pattern for a cylinder to obtain the beam pattern for a linear array of cylinders. It is worth noting that because the beam pattern for the cylinder is nearly omnidirectional, there is very little difference between the beam pattern for a linear array of point receivers and the beam pattern for a linear array of 
cylinders. This can be seen in Figure 14 for a linear array with 7/8 wavelength spacing between elements.

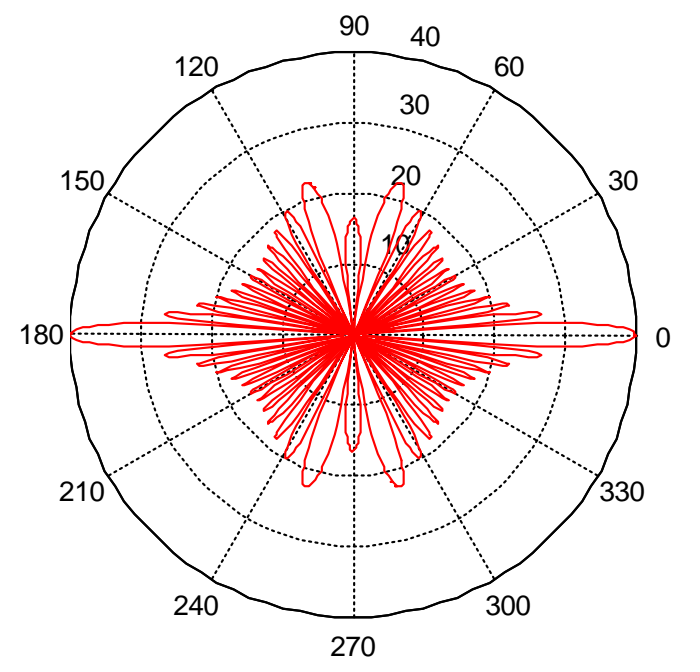

Array of point recievers (Horizontal DI $=15.65 \mathrm{~dB}$ )

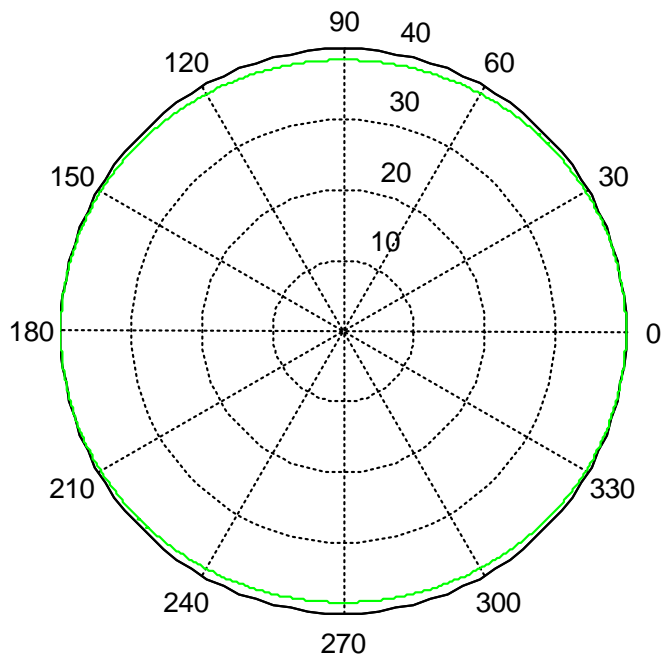

Cylinder (Horizontal $\mathrm{DI}=0.74 \mathrm{~dB}$ )

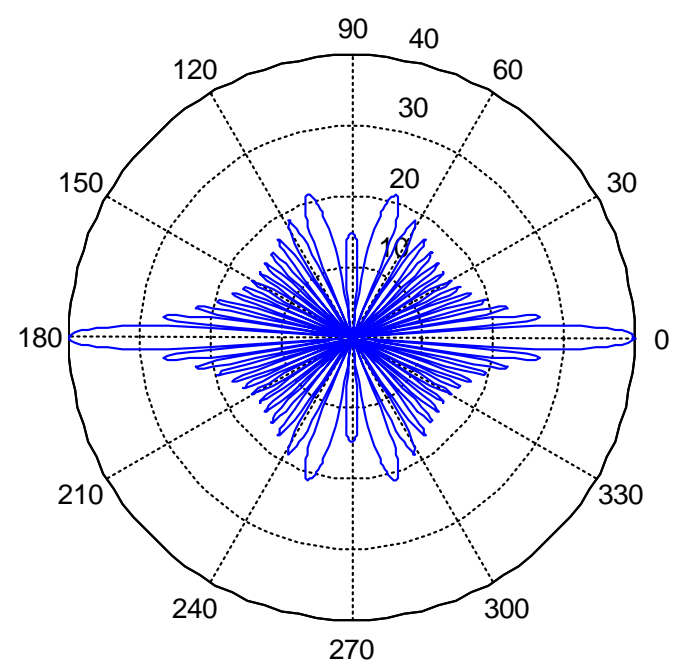

Array of cylinders (Horizontal DI $=15.77 \mathrm{~dB}$ )

Figure 14 Beam patterns for a linear array of 15 point receivers, a single cylinder, and a linear array of 15 cylinders (for 7/8 wavelength spacing) 


\subsection{Final Array Design}

The cylinders are free-flooded (oil filled) for deep submergence. The rubber rings are used to isolate the cylinders from the stainless steel rods (Figure 15). The rest of the design decisions incorporate standard linear array technology.

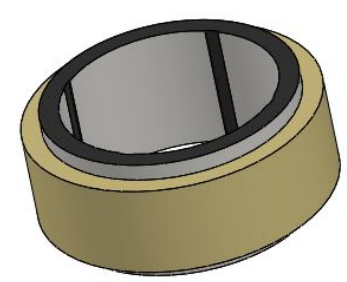

Figure 15 Cylinder and rubber ring

The final multimode linear hydrophone array design is illustrated in Figure 16. Table 6 contains the dimensions.

Table 6 Array measurements

\begin{tabular}{lc}
\hline \hline & Measurement (cm) \\
\hline Array length & 71.0 \\
Array diameter & 4.8 \\
Steel rod length & 61.0 \\
Steel rod diameter & 0.3 \\
Brass endcap length & 2.1 \\
Brass endcap diameter & 3.7 \\
Cylinder ID & 2.2 \\
Cylinder OD & 2.5 \\
Rubber ring ID & 2.5 \\
Rubber ring OD & 3.0 \\
Center-to-center cylinder spacing & 3.5 \\
Rubber boot ID & 3.8 \\
Rubber boot OD & 4.8 \\
\hline \hline
\end{tabular}




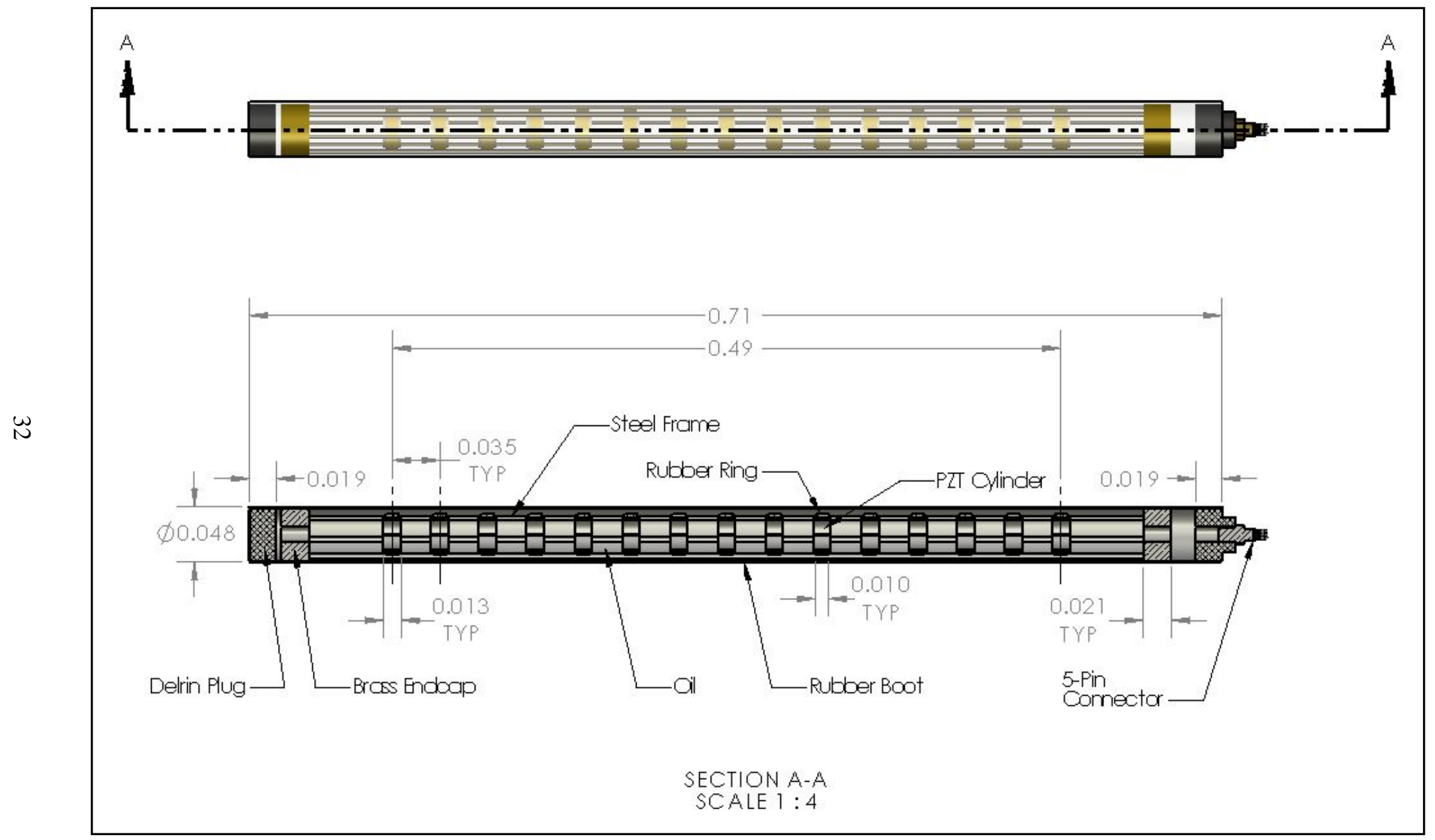

Figure 16 Multimode linear hydrophone array design (all dimensions are in meters) ${ }^{5}$

${ }^{5}$ SOLIDWORKS drawings courtesy of Dr. William Martin, NUWC, Newport, RI 


\section{CHAPTER 4 THEORETICAL (PREDICTED) DIRECTIVITY}

Both the 3-D and the 2-D theoretical (predicted) beam patterns and directivity indexes are presented in this chapter. The 3-D theoretical beam pattern and directivity index describe the potential performance that can be achieved. However, because actual measurements can only be made in two dimensions, the 2- $\mathrm{D}$ theoretical beam patterns are what the actual results are compared to. The theoretical beam patterns and directivity indexes for both the single-cylinder prototype and the 15-cylinder linear hydrophone array are described in this chapter.

\subsection{Single-Cylinder Multimode Prototype}

To calculate the 3-D beam pattern of a multimode (cardioid) sensor, the product theorem is applied to the 3-D beam pattern for a true cardioid from Equation (23) and the 3-D beam pattern for the cylinder from Equation (5) as follows:

$$
\mathrm{b}(\emptyset, \theta)=\left[\frac{1+\cos \emptyset \sin \theta}{2}\right]^{2}\left[\frac{\sin [(\pi \mathrm{L} / \lambda) \sin \emptyset]}{(\pi \mathrm{L} / \lambda) \sin \emptyset} \cdot \sin \theta\right]^{2}
$$

This 3-D beam pattern is shown in Figure 17.
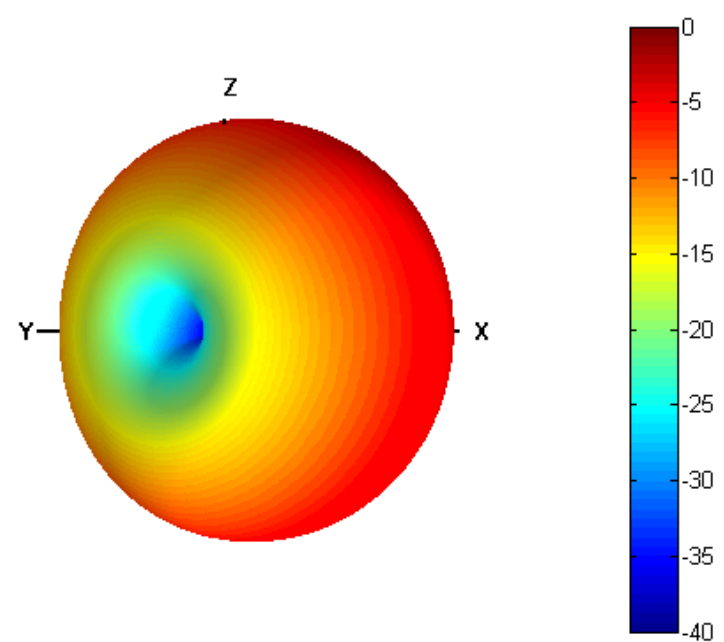

Figure 17 Theoretical 3-D beam pattern for multimode cylinder (DI=5.2 dB) 
Applying Equation (15) gives a cardioid cylinder 3-D directivity index of $5.2 \mathrm{~dB}$. Note that the beam pattern and directivity index for the cardioid cylinder are only slightly different than those for a true cardioid (Figure 8), because the cylinder is almost omnidirectional.

Taking a slice of the above $3 \mathrm{D}$ shape at theta equal to 90 degrees provides the horizontal beam pattern. Likewise, taking a slice at phi equal to zero degrees provides the vertical beam pattern. These beam patterns and their respective 2-D directivity indexes are shown in Figure 18.

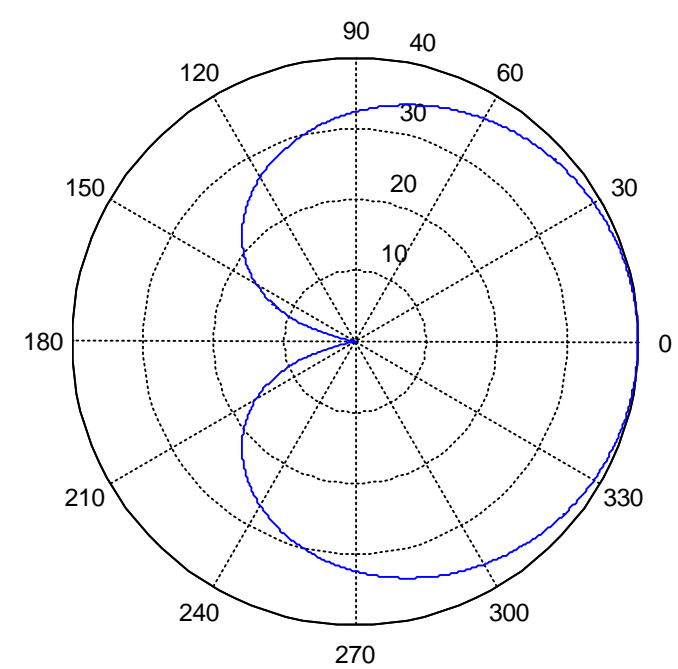

(a) Theoretical horizontal beam pattern (xy plane) (horizontal DI $=4.87 \mathrm{~dB}$ )

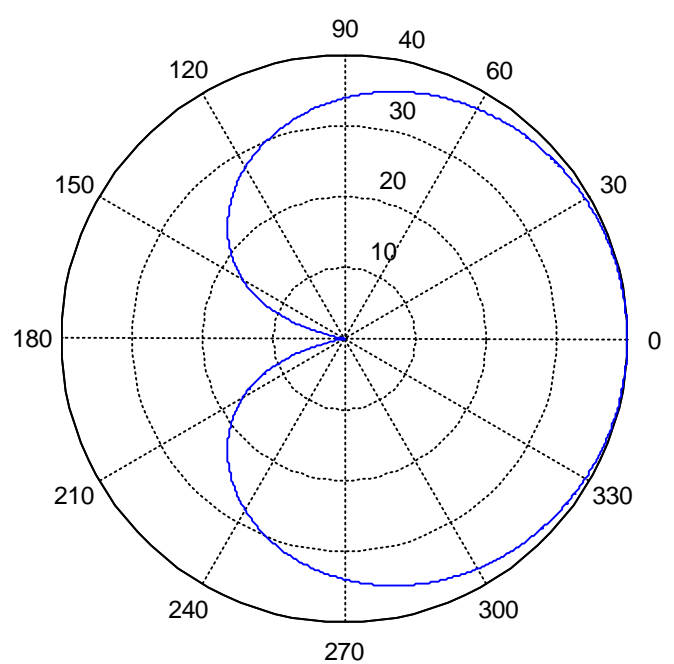

(b) Theoretical vertical beam pattern (xz plane) (vertical DI $=4.26 \mathrm{~dB}$ )

Figure 18 Theoretical 2-D multimode cylinder beam patterns 


\subsection{Multimode Linear Hydrophone Array}

The theoretical 3-D beam pattern for a linear array of 15 multimode cylinders, according to the product theorem, is the product of the 3-D beam pattern for the multimode cylinder which is given by Equation (35) and shown in Figure 17, and the 3-D beam pattern for a linear array of 15 isotropic sensors which is given by Equation (10) and shown in Figure 19.
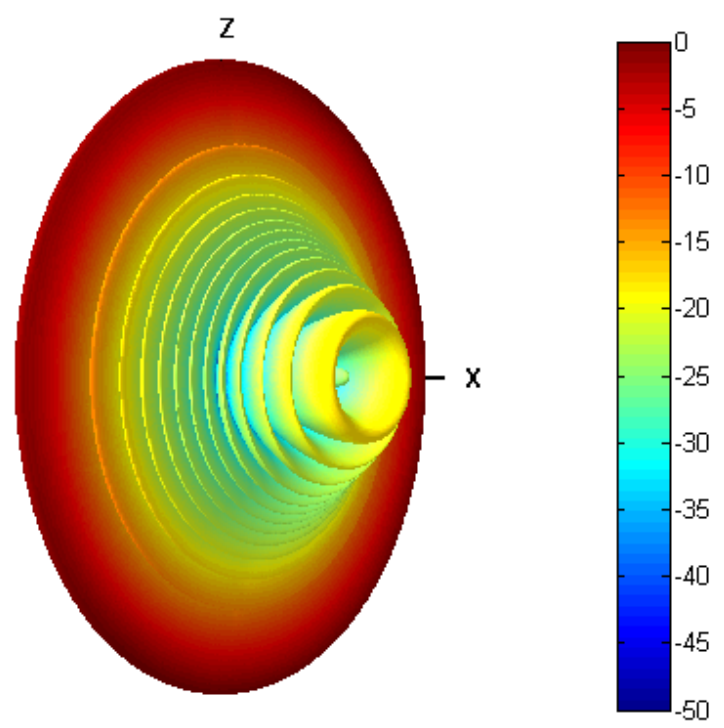

Figure 19 Theoretical 3-D beam pattern for linear array of 15 isotropic sensors $(\mathrm{DI}=14.0 \mathrm{~dB})$

The 3-D beam pattern for a linear array of multimode cylinders becomes:

$$
\mathrm{b}(\emptyset, \theta)=\left[\frac{1+\cos \emptyset \sin \theta}{2}\right]^{2}\left[\frac{\sin [(\pi \mathrm{L} / \lambda) \sin \emptyset]}{(\pi \mathrm{L} / \lambda) \sin \emptyset} \cdot \sin \theta\right]^{2}\left[\frac{1}{\mathrm{~N}} \sum_{\mathrm{n}=0}^{\mathrm{N}-1} \mathrm{e}^{\mathrm{jnkd} \cos \emptyset \sin \theta}\right]^{2}
$$

Multiple views of the resulting beam pattern are shown in Figure 20. Applying Equation (15) gives a 3-D Directivity Index (DI) of 18.35. This is the directivity index that is used in Chapter 12 to calculate the predicted detection range for the 15-cylinder multimode hydrophone array. 

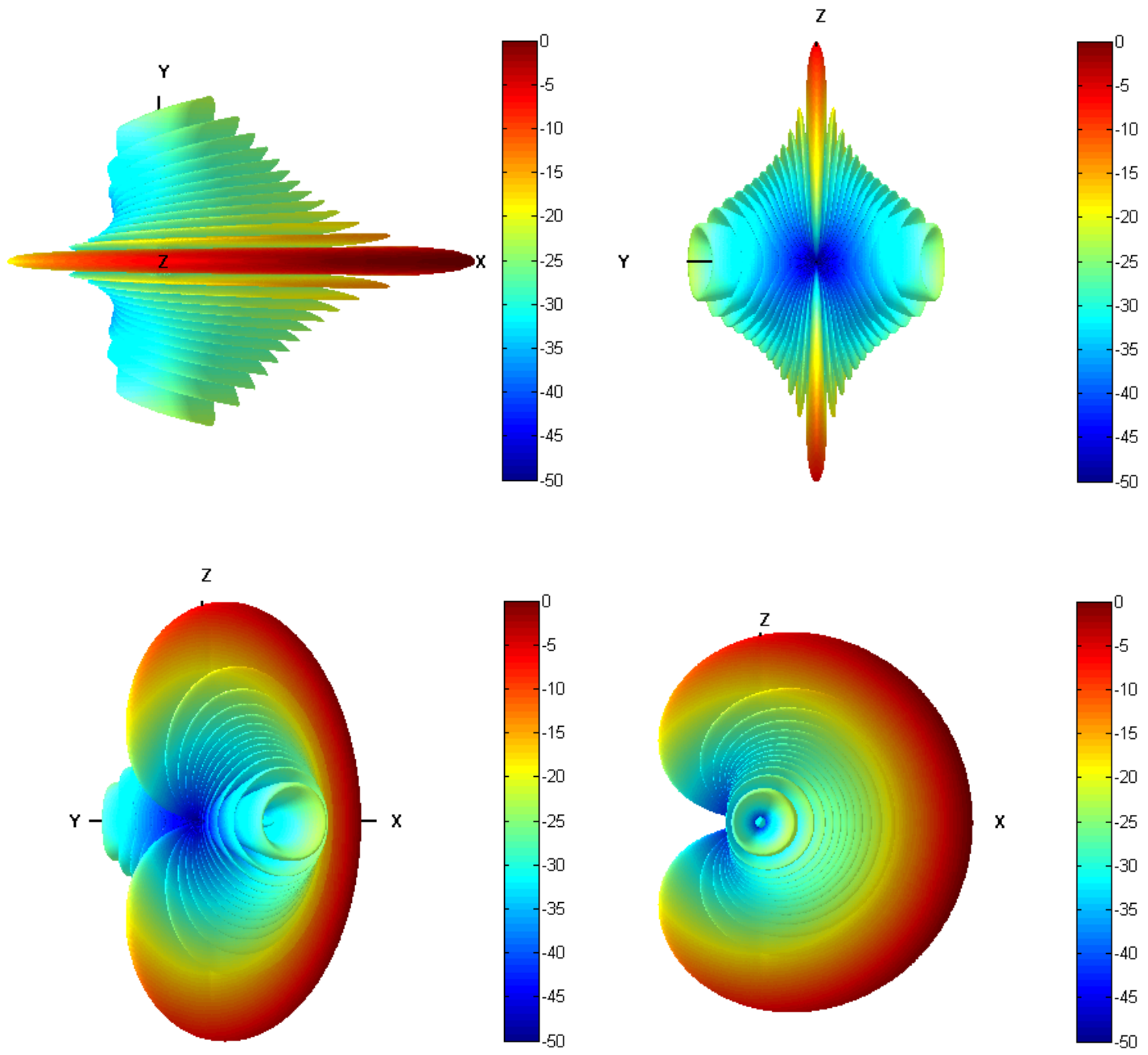

Figure 20 Theoretical 3-D beam pattern for linear array of 15 multimode cylinders (DI=18.35 dB)

Again, taking a slice of the above 3-D shape at theta equal to 90 degrees provides the horizontal beam pattern, and taking a slice at phi equal to zero degrees provides the vertical beam pattern. These beam patterns and their respective 2-D directivity indexes are shown in Figure 21. 


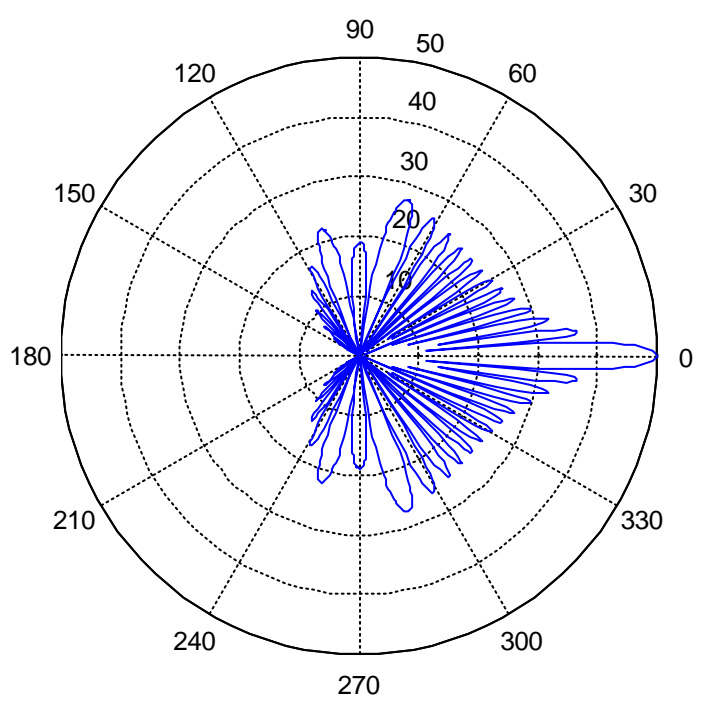

(a) Theoretical horizontal beam pattern (xy plane) (horizontal DI = $18.9 \mathrm{~dB}$, beam width $=4.3$ degrees)

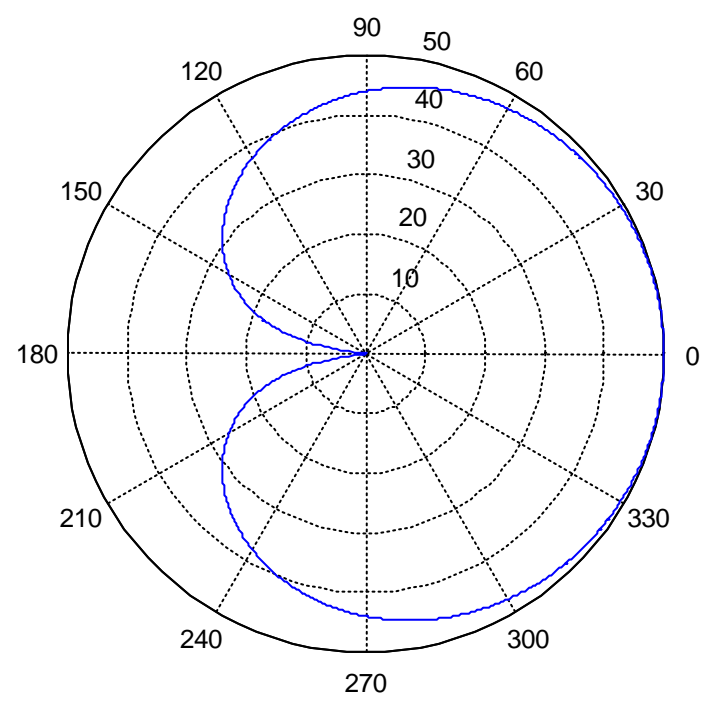

(b) Theoretical vertical beam pattern (xz plane) (vertical DI $=4.3 \mathrm{~dB}$, beam width $=131.8$ degrees)

Figure 21 Theoretical 2-D beam patterns for linear array of 15 multimode cylinders 


\section{CHAPTER 5 IN-AIR CHARACTERIZATION}

When fabricating acoustic apertures, it is common practice to first perform in-air characterizations of the individual transducers. The purpose for doing so is to establish a baseline of performance and to identify any variation that may exist from one element to the next. Typical in-air measurements include admittance, blocked capacitance, leakage resistance and dissipation factor.

For this thesis, in-air admittance, blocked capacitance, leakage resistance and dissipation factor measurements were made on each of the four quadrants of each of the 15 cylinders that were used in the array. In this chapter, these measurements are presented, and compared to the manufacturer's specification when available.

\subsection{Admittance}

Admittance, $Y$, is the ratio of input current to applied voltage with the output open-circuited. "Open-circuit" implies that the mechanical side is constrained, or clamped, so that it is not free to move, and the displacement, and therefore the strain in the material, is zero. This is achieved with a static applied voltage or with a low frequency applied sinusoidal voltage (Burdic, 1991).

The admittance of each quadrant of each of the 15 cylinders was measured using an Admittance Gain Phase Analyzer. The measurements were made over a range of frequencies near the cylinder's in-water resonance frequency of $42 \mathrm{kHz}$. Figure 22 shows the measured admittance magnitude in milliSiemens $\left(10^{-12}\right.$ Siemens) and phase in degrees for all four quadrants of one cylinder (typical of each cylinder). Figure 23 shows the measured admittance magnitude and phase for one quadrant (typical of each quadrant) for all 15 cylinders. From the plots, it can be seen that there is not much 
variance from one quadrant to another on a given cylinder, but there is a slight variance from one cylinder to another. Admittance magnitude peaks at the resonance frequency of the transducer. From the plots it can be seen that the measured in-air resonance frequencies range from $45.5 \mathrm{kHz}$ to $46.5 \mathrm{kHz}$, slightly higher than the manufacturer's specified in-water resonance frequency of $42 \mathrm{kHz}$.
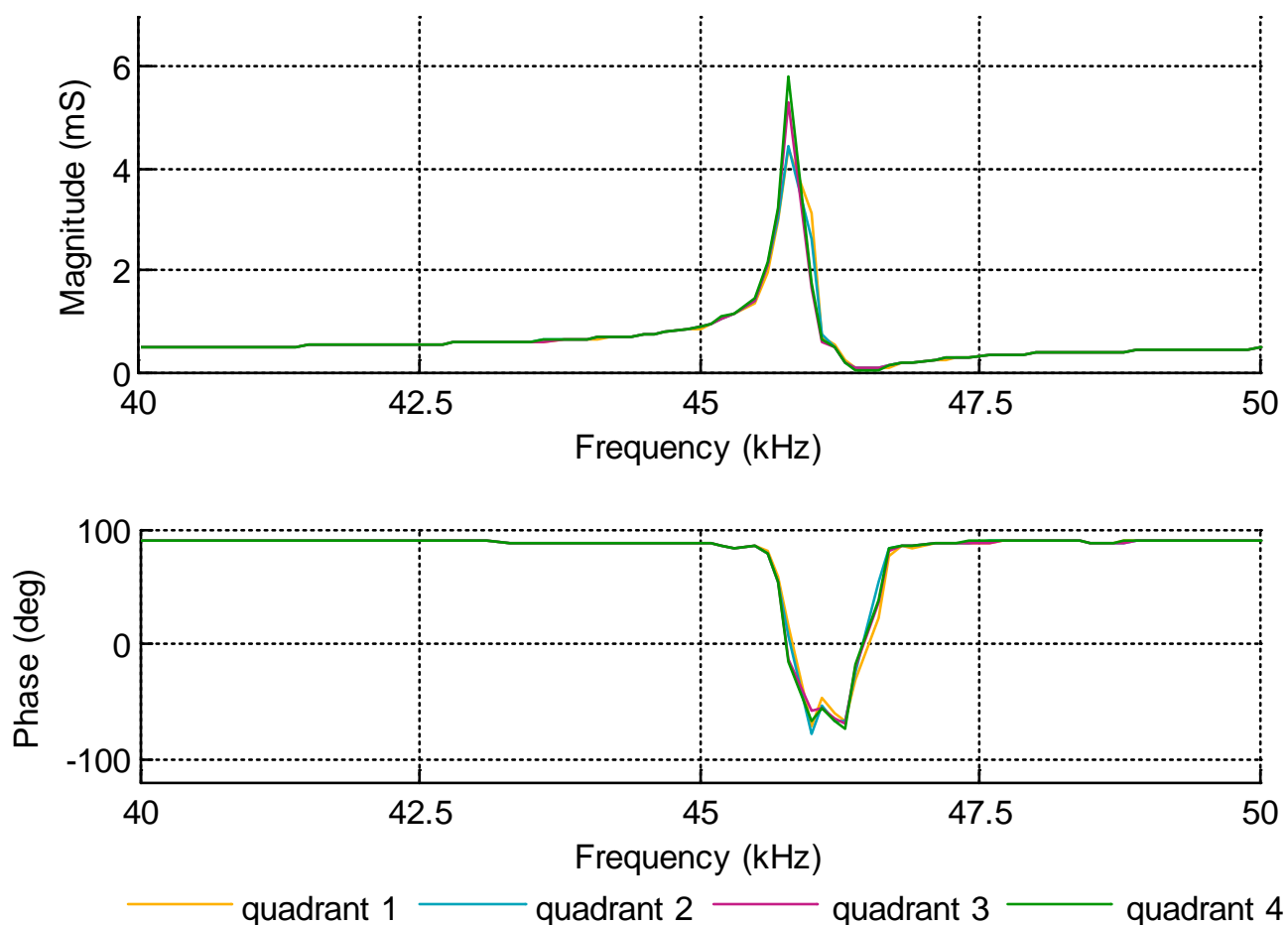

Figure 22 In-air admittance for all four quadrants of cylinder 15 

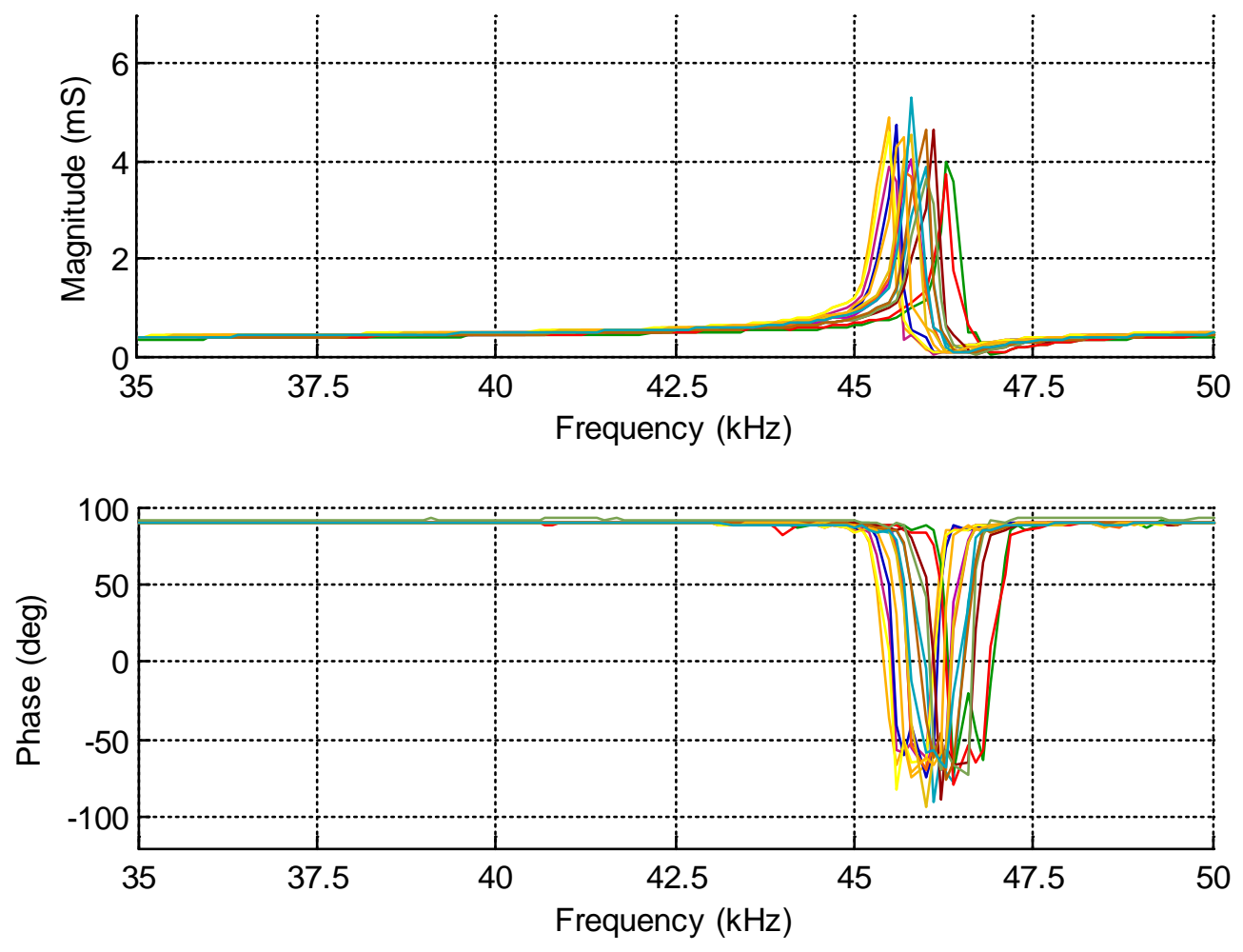

Figure 23 In-air admittance for quadrant 3 of all 15 cylinders

\subsection{Blocked Capacitance}

Blocked capacitance, also referred to as the clamped or static capacitance, is the capacitance in a piezoelectric material under the clamped condition, when no strain (deformation) is permitted. The manufacturer specifies a blocked capacitance of 6600 pF +/- 20\% @ 1kHz. Blocked capacitance of each quadrant of each of the 15 cylinders was measured with an LCR Meter at $1 \mathrm{kHz}$. Figure 24 shows the measured blocked capacitance in picoFarads ( $10^{-12}$ Farads) for all four quadrants of each of the 15 cylinders. 


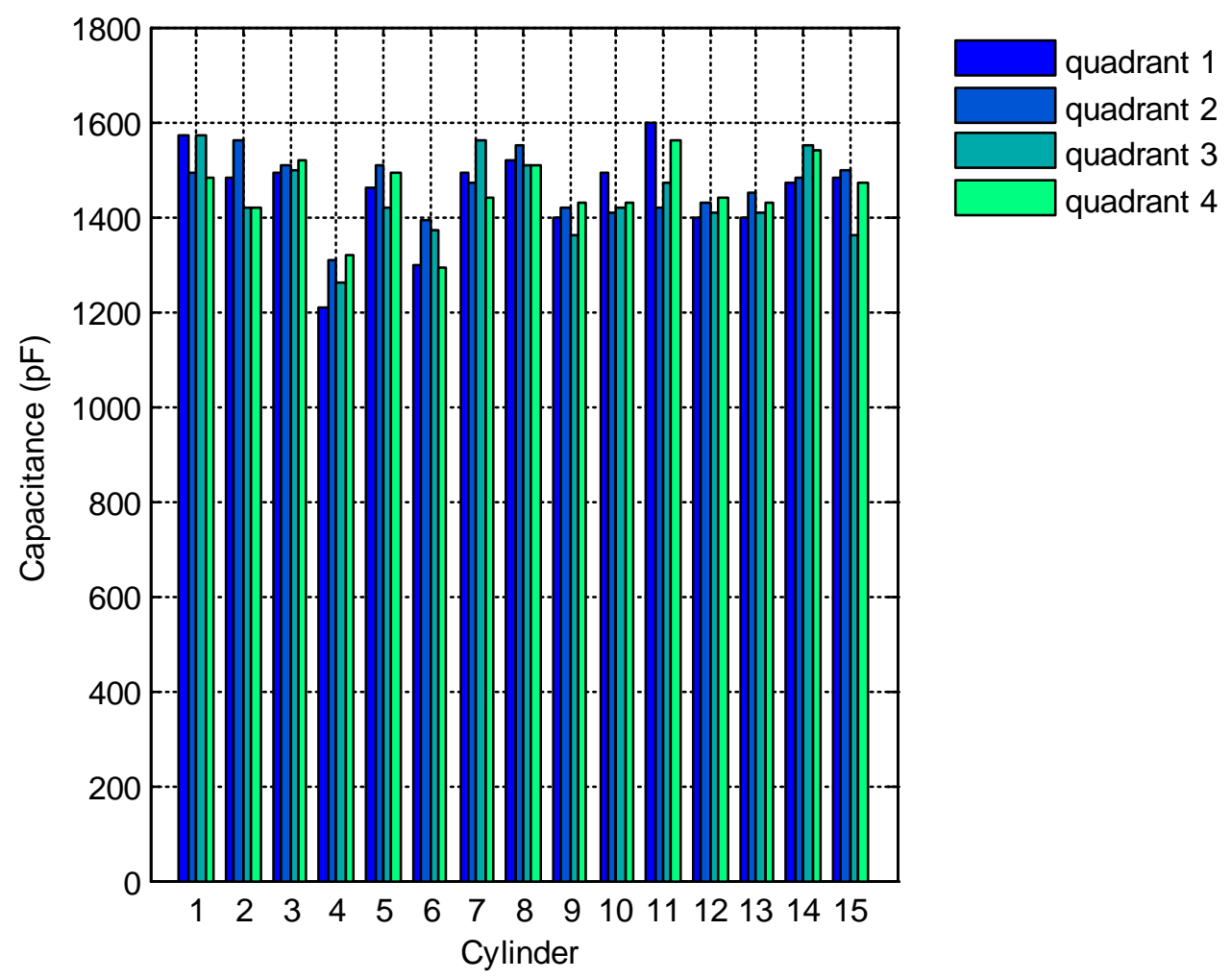

Figure 24 In-air blocked capacitance for all cylinders and segments

\subsection{Leakage Resistance}

Leakage resistance, also referred to as insulation resistance, is the extent to which a dielectric material resists leakage current. In piezoelectric ceramics, a high number such as 10 Gigaohms is desired. The leakage resistance of each quadrant of each of the 15 cylinders was measured with a Megohmmeter. Figure 25 shows the measured leakage resistance in Gigaohms $\left(10^{9} \mathrm{Ohms}\right)$ for all four quadrants of each of the 15 cylinders. 


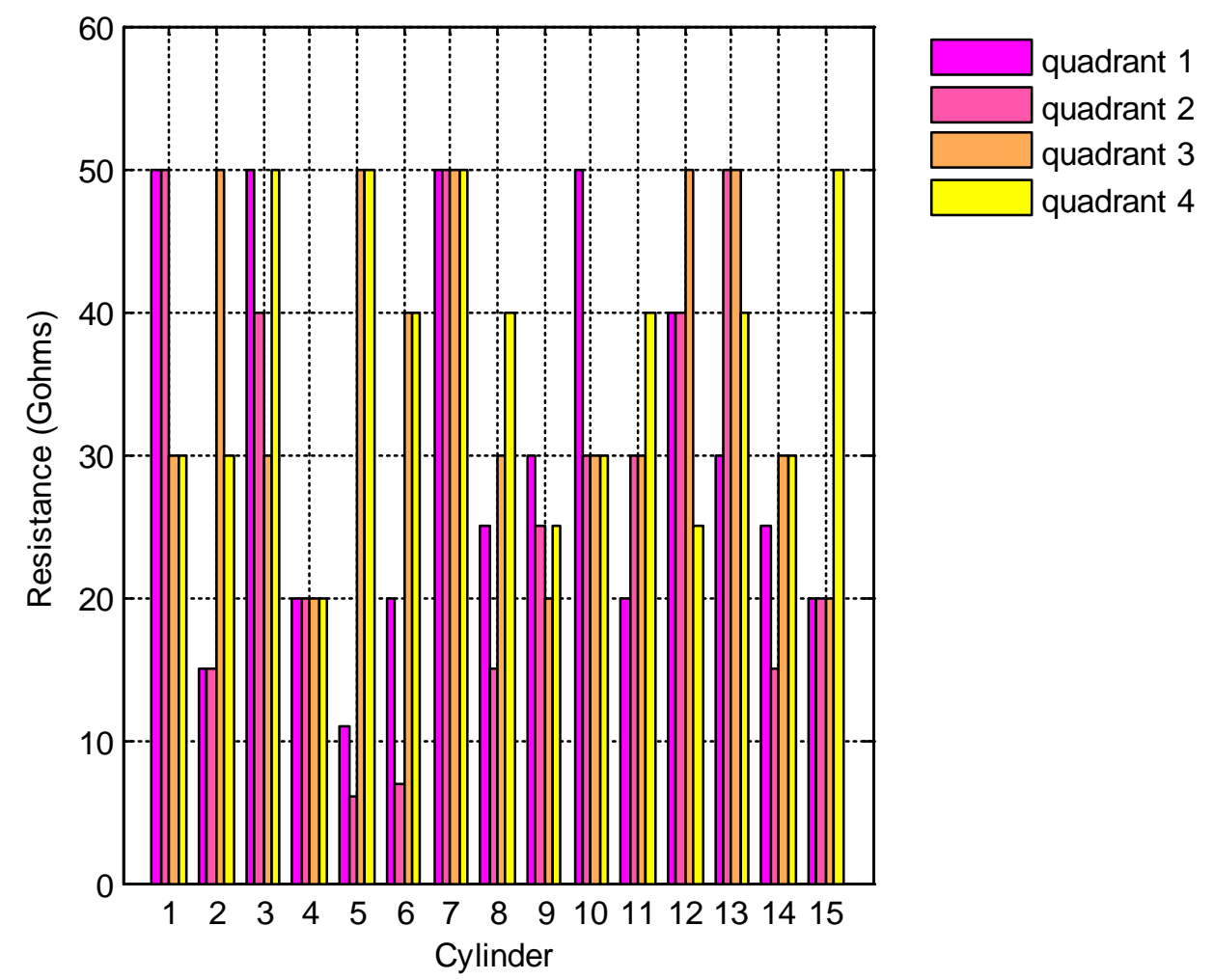

Figure 25 In-air leakage resistance for all cylinders and segments

\subsection{Dissipation Factor}

Dissipation factor is a measure of the losses in a capacitor and is sometimes called the "loss tangent". It is the ratio of the equivalent series resistance to the capacitive reactance, and is usually expressed as a percentage. The reciprocal of the dissipation factor is called the "Q”, or quality factor. The manufacturer specifies a dissipation factor at $1 \mathrm{kHz}$ of $0.5 \%$. The dissipation factor of each quadrant of each of the 15 cylinders was measured at $1 \mathrm{kHz}$ with an Inductance, Capacitance, and Resistance (LCR) Meter. Figure 26 shows the measured dissipation factor percentage for all four quadrants of each of the 15 cylinders. 


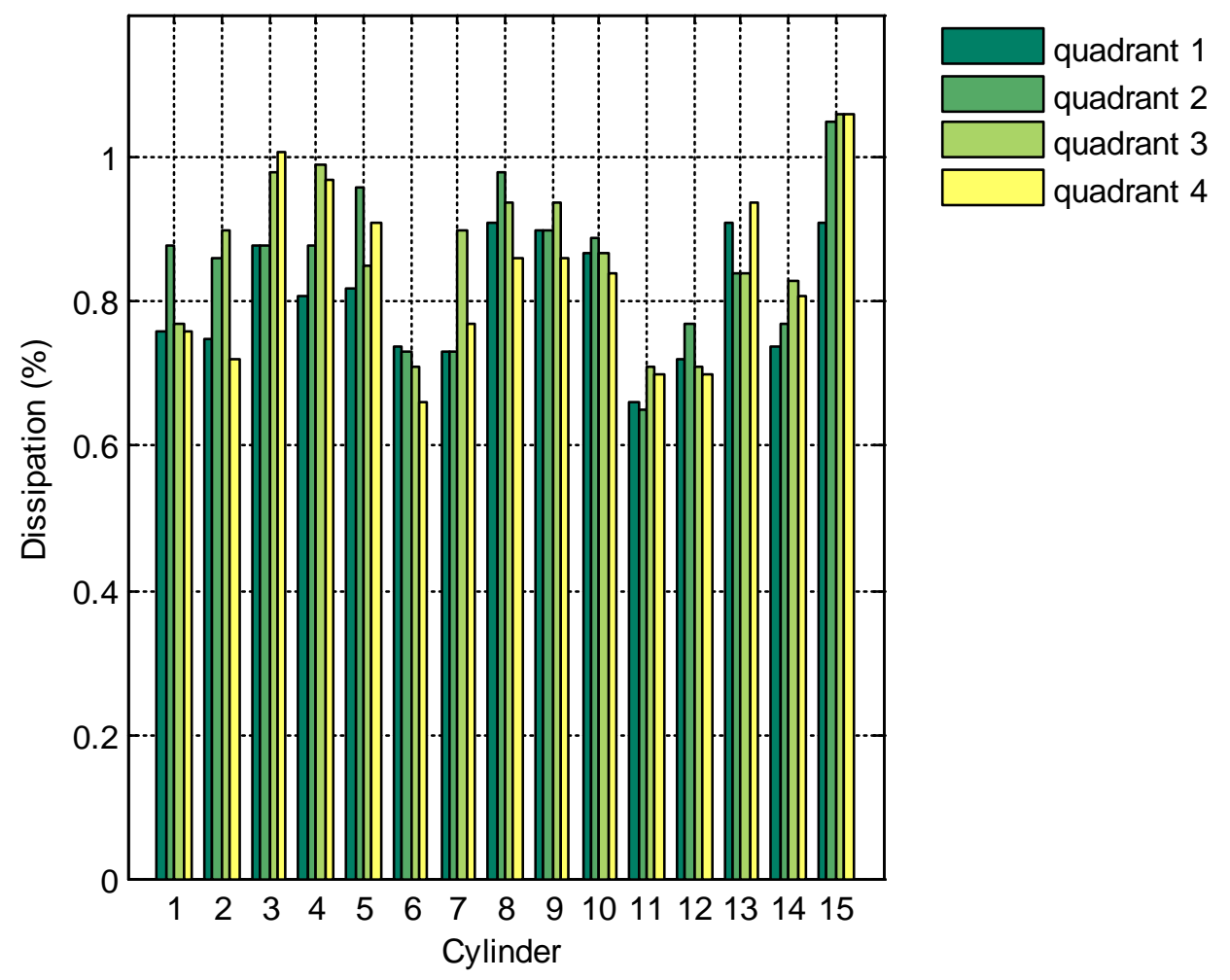

Figure 26 In-air dissipation factor for all cylinders and segments 


\section{CHAPTER 6 HYDROPHONE PROTOTYPE FABRICATION}

As previously mentioned, a single-cylinder hydrophone prototype was first fabricated. Table 7 lists the materials used to build the single-cylinder hydrophone.

Table 7 Hydrophone Material List

\begin{tabular}{|c|c|}
\hline Quantity & Description \\
\hline 1 & $\begin{array}{l}\text { STEMiNC Piezoelectric ceramic (modified PZT-4) } \\
\text { striped } 4 \text { quadrant cylinder, } 42 \text { kHz, radially poled, } \\
\text { 26mm OD x } 22 \text { mm ID x } 13 \text { mm length, part number } \\
\text { SMC2622T13111SP }\end{array}$ \\
\hline 1 & 5-pin connector \\
\hline \multirow[t]{5}{*}{$15 / 8 ”$} & 1 3/4" inch diameter Delrin plastic rod \\
\hline & Heat-shrink plastic tubing \\
\hline & Solder \\
\hline & Soldering flux \\
\hline & Polyurethane potting compound \\
\hline
\end{tabular}

The single-cylinder hydrophone prototype was constructed as follows:

A hollow was machined in the Delrin plastic rod, leaving $1 / 4$ inches of material on the sides and on one end. A 7/16” threaded hole was machined into the closed end, and the 5-pin connector was screwed into the hole. The four signal wires were soldered to the four inside electrodes of the piezoelectric cylinder. The ground wire was soldered to the outside of the cylinder. The cylinder and wires were pushed into the Delrin housing and the cylinder was centered. The potting compound was poured into the housing while taking care to keep the cylinder centered. The potting compound was cured at room temperature. Figure 27 includes photos of the inprocess product. Figure 28 includes a photo of the final product. 

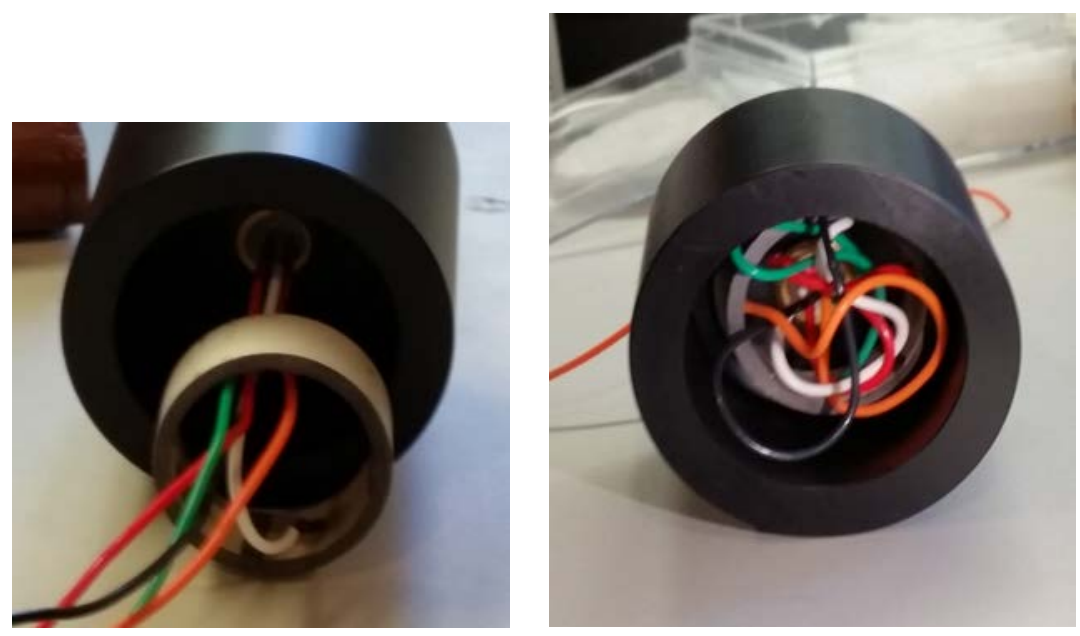

Figure 27 Fabrication of the single-cylinder hydrophone

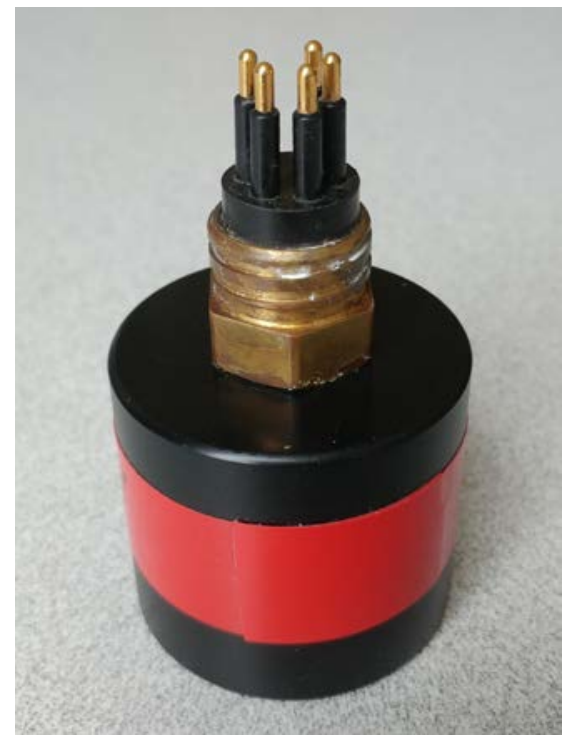

Figure 28 Final single-cylinder hydrophone 


\section{CHAPTER 7 HYDROPHONE PROTOTYPE TESTING}

On October 14, 2016, the single-cylinder hydrophone was tested in the Open Tank Facility (OTF) at the Naval Undersea Warfare Center (NUWC), Newport, RI.

The purpose of the test was two-fold: 1) to characterize the performance of a single-cylinder multimode hydrophone, and 2) to provide data that could be used to test the MATLAB code being written to generate the cardioid beam pattern from the four channels of measured voltages.

\subsection{Test Facility}

The OTF is one of the six measurement facilities maintained by the Underwater Sound Reference Division (USRD) which serves as the U.S. standardizing activity in the area of underwater acoustic measurements. The USRD is part of NUWC Division Newport's Sensor and Sonar Systems Department.

The OTF is a fresh water facility. The tank is an indoor concrete tank, 4.57 meters wide, 4.57 meters deep and 9.14 meters long. Figure 29 is a pictorial of the OTF.

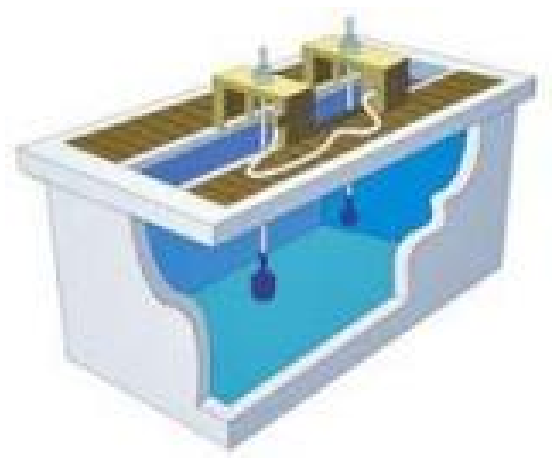

Figure 29 NUWC Open Tank Facility pictorial (Paolero and Évora, 2015)

The transducer (transmitter) and hydrophone (receiver) are attached to 1-inch diameter hollow (free-flooded) stainless steel poles and positioned laterally by a carriage and vertically by a crane. Each carriage holds a turntable which rotates the 
pole about it axis for proper positioning and directivity measurements. Figure 30 is a photo of one of the OTF's positioning systems.

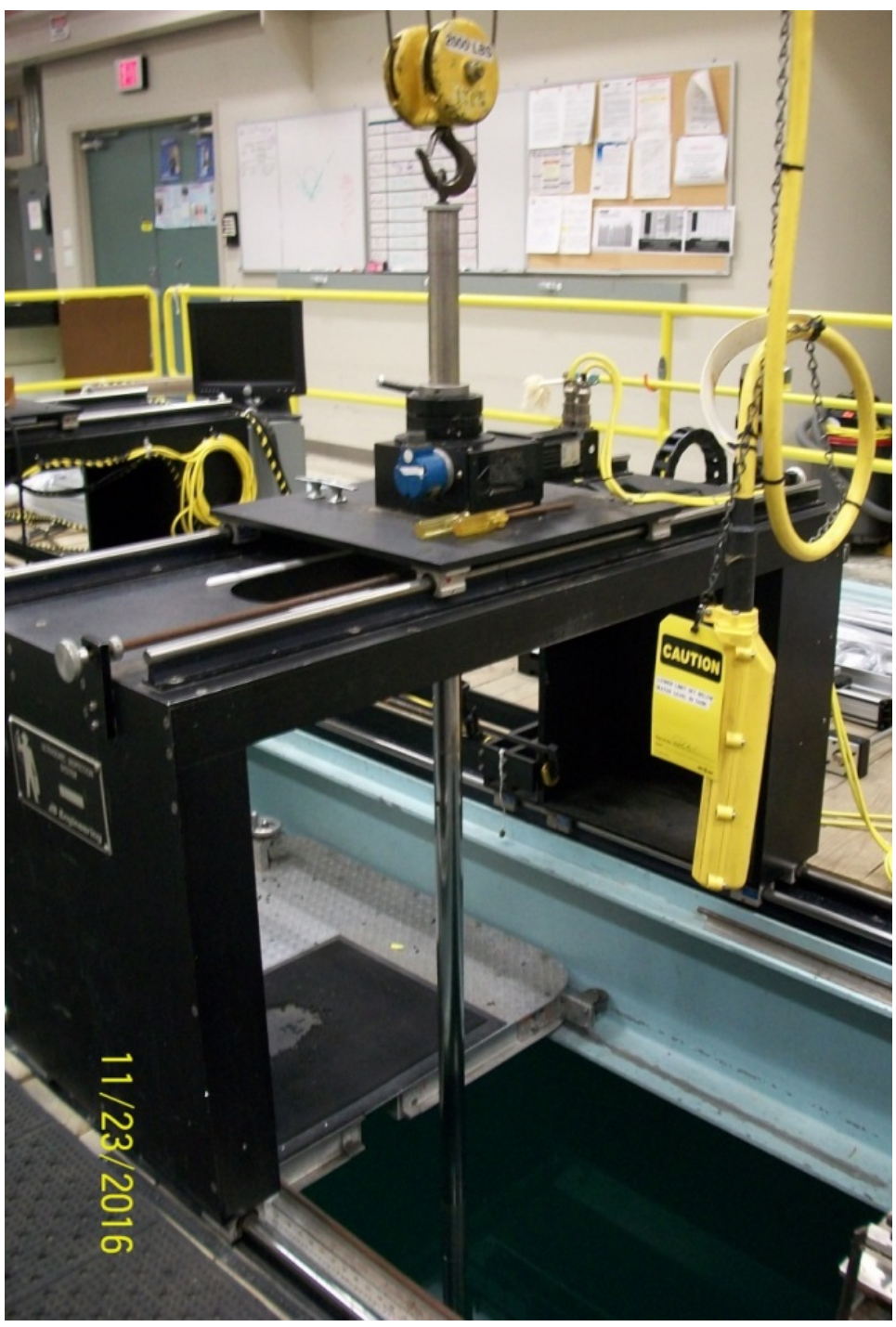

Figure 30 NUWC Open Tank Facility photo 
The test equipment is described below in Table 8 .

Table 8 Open Tank Facility Test Equipment

\begin{tabular}{|c|c|}
\hline Equipment & Description \\
\hline Test Set Hardware & $\begin{array}{l}\text { National Instruments PXI-1045 8-slot PXI } \\
\text { chassis with the following modules: } \\
\text { National Instruments PXI-8360 remote } \\
\text { control module } \\
\text { National Instruments PXI-6652 timing } \\
\text { module } \\
\text { National Instruments PXI-5412 } \\
\text { waveform generator } \\
\text { National Instruments PXI-5105 8-channel } \\
\text { oscilloscope/digitizer } \\
\text { TEGAM 4040B PXI instrumentation } \\
\text { amplifiers (1 per channel) }\end{array}$ \\
\hline Test Set Software & $\begin{array}{l}\text { THAMES (Transducer and Hydrophone } \\
\text { Acoustic Measurement and Evaluation } \\
\text { System), a LabVIEW based system }\end{array}$ \\
\hline Projector & USRD Type F83 transducer \\
\hline
\end{tabular}

\subsection{Test Setup}

A cable assembly with four 10.4 meter coaxial cables (one for each of the cylinder's 4 quadrants), a 5 pin connector at one end and 4 crimp connectors at the other end was used. To extend the length of the cables, a 1.2 meter RG-58 cable extension was added to each. The cable extensions were connected to four test set input channels. Input channel 1 corresponded to quadrant 1 of the PZT cylinder, input channel 2 corresponded to quadrant 2, etc.

The 5 pin connector on the hydrophone was connected to the mating 5 pin connector on the cable. The hydrophone was then mounted to a 1-inch diameter hollow stainless steel pole which was attached to a rolling carriage with a turntable, 
and lowered into the tank to the same depth as the projector, 90 inches below the water surface. The hydrophone was positioned 2 meters from the projector.

For each test, the projector transmitted an acoustic signal at a specified frequency, and in response, the hydrophone generated four analog low-level voltage signals (one for each of the multimode cylinder quadrants) proportional to the acoustic pressure acting upon the hydrophone. An analog-to-digital (A/D) converter converted the analog signals into digital signals which were recorded and processed by the test set.

In order to prevent reflections from the water surface and side of the tank from interfering with the measurements, pulsed continuous waves were transmitted by the projector, and receive gates were used by the test set to exclude the reflections.

The specific environmental conditions and test set parameters are described below in Table 9.

Table 9 Test Conditions October 14, 2016

\begin{tabular}{ll}
\hline \hline Parameter & Value \\
\hline $\begin{array}{l}\text { Separation distance between } \\
\text { projector and hydrophone }\end{array}$ & 2 meters \\
$\begin{array}{l}\text { Depth of projector and } \\
\text { hydrophone }\end{array}$ & $2.28 \mathrm{~m}$ (90 inches) \\
Transmit frequency & $\begin{array}{l}37.5 \mathrm{kHz} \text { (the designed operating } \\
\text { frequency of the hydrophone) }\end{array}$ \\
Water temperature & 19.7 degrees C \\
Sound speed & $1481 \mathrm{~m} / \mathrm{s}$ \\
Bearing resolution & 1.5 degrees \\
Sampling rate & $5 \mathrm{MHz}$ \\
Pulse duration & $1.2 \mathrm{~ms}$ \\
\hline \hline
\end{tabular}




\subsection{Test Procedure}

Free field voltage sensitivity (FFVS) readings were first taken. Free field voltage sensitivity is one of the measurements used to predict the performance of an acoustic aperture. Free field voltage sensitivity is the ratio of the complex voltage output by the aperture to the complex pressure acting on the aperture when in the presence of an undisturbed sound pressure wave in a free field. Free field voltage sensitivity is therefore a complex quantity with both a magnitude and a phase.

“Free field” implies that the sound may propagate free from any form of obstruction. The basic requirements for "free field" measurements are (1) enough space so that interference from boundary reflections can be eliminated by pulsing techniques, anechoic boundaries, or spreading losses due to long distances, (2) a low ambient noise level, and (3) a water medium that is relatively free of anything that will cause refraction and scattering (Bobber, 1988).

The THAMES test set computes free field voltage sensitivity using a comparison replacement calibration method in which a calibrated reference standard hydrophone with known free field voltage sensitivity is used to determine the sound pressure level in the water, then the hydrophone with unknown sensitivity is subjected to the known sound pressure level and computations are performed to deduce its free field voltage sensitivity (Acoustical Society of America, 2012). The THAMES test set computes the FFVS magnitudes expressed in decibels relative to one volt per micropascal (e.g. $\mathrm{dB}$ re $1 \mathrm{~V} / \mathrm{uPa}$ ), but does not compute the FFVS phases. 
For the first set of tests the hydrophone was mounted vertically to the pole (Figure 31).

Using the turntable, the hydrophone was rotated about its central axis. These measurements are referred to herein as the hydrophone vertical measurements.

While the hydrophone was stationary, FFVS measurements were taken over a range of transmit frequencies from $10 \mathrm{kHz}$ to $100 \mathrm{kHz}$. Then the hydrophone was rotated and FFVS measurements were taken at each bearing for the operating frequency of $37.5 \mathrm{kHz}$. FFVS measurements were made for each of the 4 hydrophone quadrants.

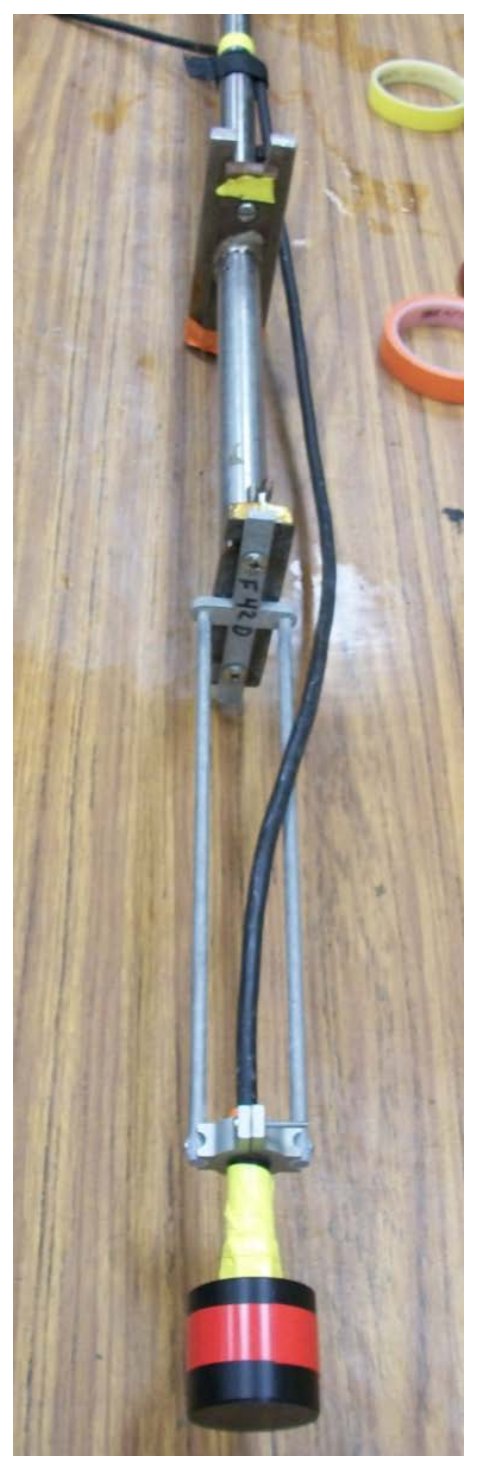

Figure 31 Hydrophone vertical test fixture

Directivity measurements were then taken. At each rotation angle, the time series voltage data was recorded for each of the four (4) quadrants. For each rotation angle, the test set calculated the voltage magnitude and phase from the time series voltage data using a Fourier transform. 
For the second set of tests, the hydrophone was mounted horizontally to the pole (Figure 32). Using the turntable, the hydrophone was rotated about its acoustic center. The same measurements were taken as for the previous orientation. These measurements are referred to herein as the hydrophone horizontal measurements.

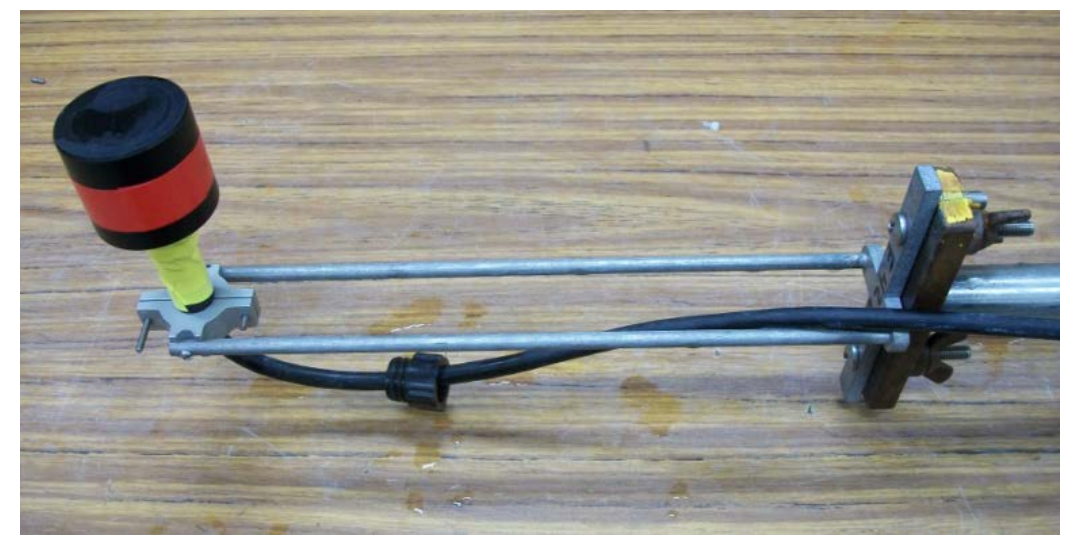

Figure 32 Hydrophone horizontal test fixture 


\section{CHAPTER 8 PROCESSING THE HYDROPHONE TEST RESULTS}

\subsection{Free Field Voltage Sensitivity}

Free field voltage sensitivity (FFVS) magnitudes were provided by the test set for each of the four quadrants over a range of frequencies. Figure 33 contains a plot of the FFVS magnitudes for each quadrant for frequencies from $10 \mathrm{kHz}$ to $60 \mathrm{kHz}$.

FFVS plots typically peak at the resonance frequency. For this cylinder, the resonance frequency is $42.0 \mathrm{kHz}$. The plot shows a peak slightly below this resonance frequency and closer to the operating frequency of $37.5 \mathrm{kHz}$.

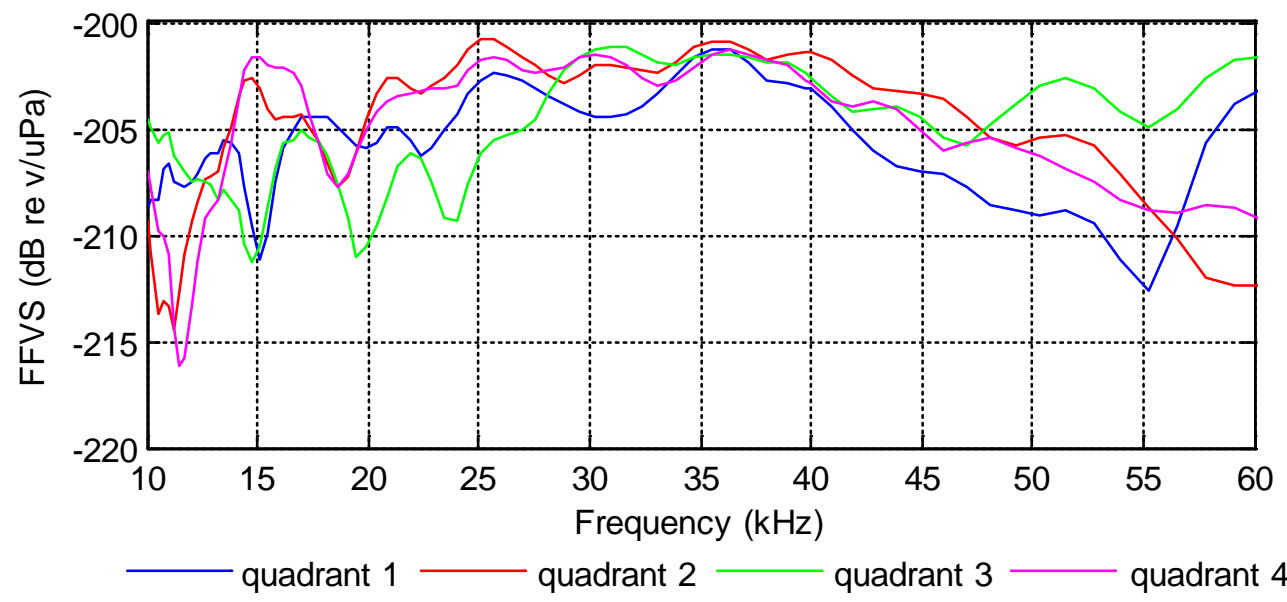

Figure 33 FFVS magnitude vs. frequency for single-cylinder hydrophone

Table 10 lists the FFVS magnitudes for each of the hydrophone's four quadrants at the operating frequency of $37.5 \mathrm{kHz}$.

Table 10 Hydrophone FFVS Magnitude at $37.5 \mathrm{kHz}$

\begin{tabular}{cc}
\hline \hline Quadrant & $\begin{array}{c}\text { FFVS @ } 37.5 \mathrm{kHz} \\
(\mathrm{dB} \text { re } 1 \mathrm{~V} / \mu \mathrm{Pa})\end{array}$ \\
\hline 1 & -202.3 \\
2 & -201.5 \\
3 & -201.8 \\
4 & -201.6 \\
\hline \hline
\end{tabular}




\subsection{Vertical Directivity}

From the voltage time series data that was recorded for each quadrant while the hydrophone was rotated in its vertical orientation, the test set calculated the complex voltage (magnitudes and phase) for each quadrant, at each angle of arrival. Figure 34 shows how the voltage magnitudes and phases varied from one quadrant to the next as the hydrophone was rotated.
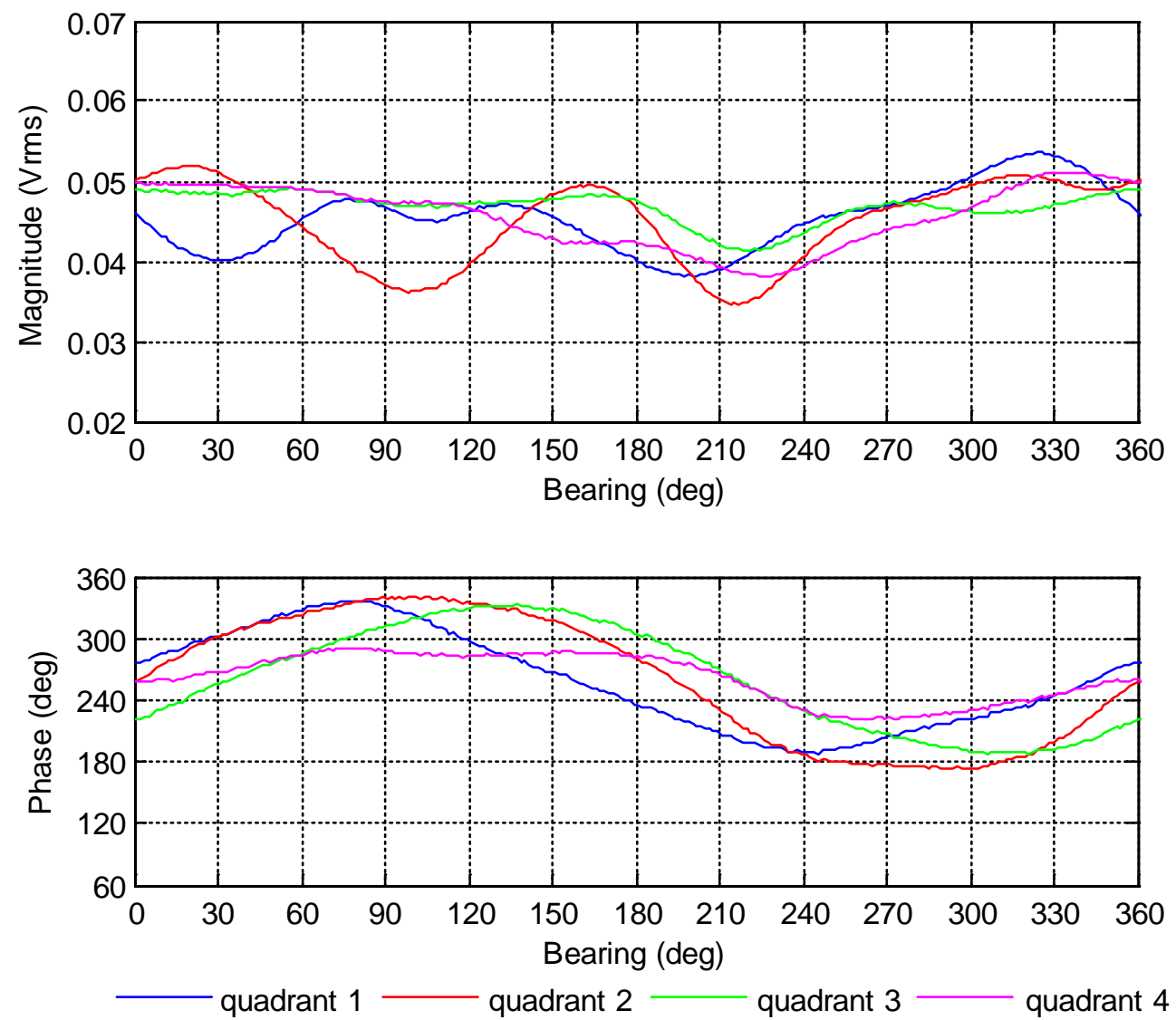

Figure 34 Hydrophone voltage magnitudes and phases at each angle in the vertical (xz) plane

The complex voltages for quadrants 1 through $4, V_{1}, V_{2}, V_{3}$ and $V_{4}$, respectively, were used in Equations (26) through (34) to calculate the actual omni, dipole and cardioid beam patterns for this vertical rotation. The values used in Equation (31), and the resulting value for $R$, are shown here: 


$$
\mathrm{R}=\frac{-\mathrm{V}_{\mathrm{O}}(\pi)}{\mathrm{V}_{\mathrm{D}}(\pi)}=\frac{-(-0.0897-0.1084 i)}{-0.0466+0.0451 i}=0.1662-2.1633 \mathrm{i}
$$

The actual (measured) omni, dipole and cardioid beam patterns for this vertical rotation are compared to the theoretical (predicted) beam patterns in Figure 35(a)-(c).

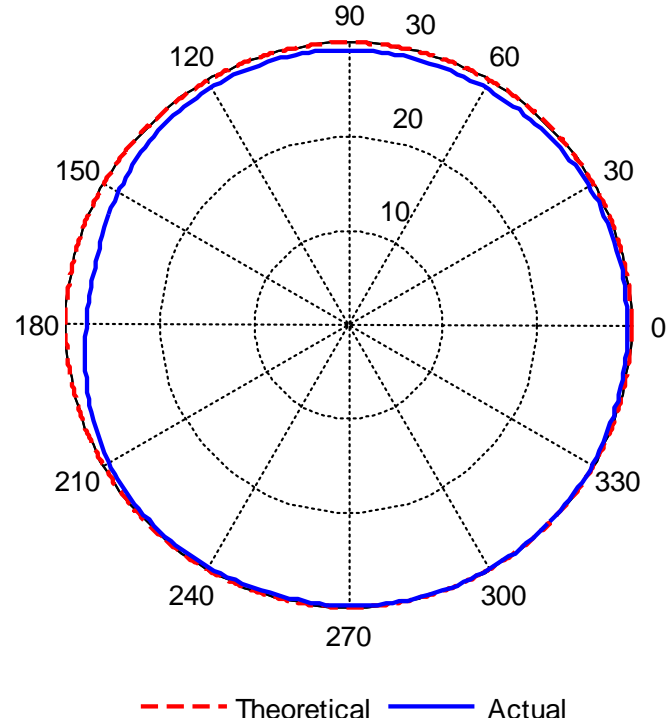

(a) Hydrophone omni beam pattern

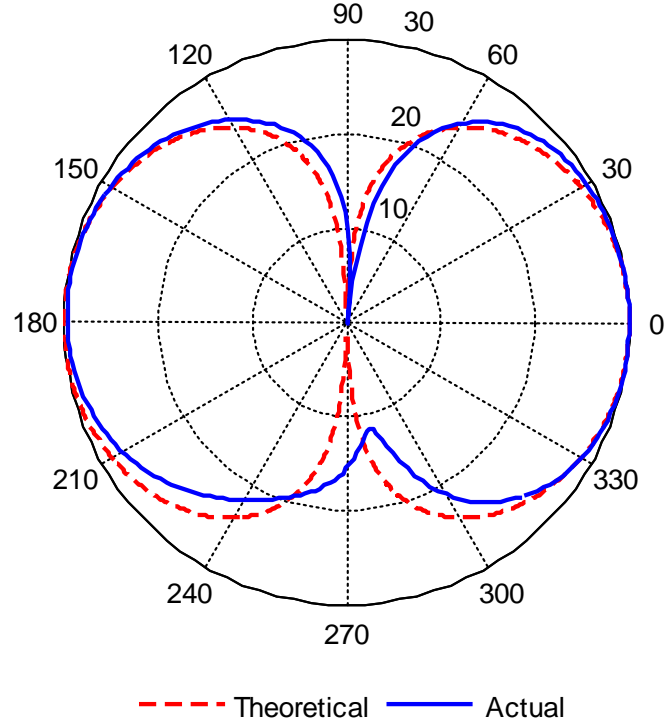

(b) Hydrophone dipole beam pattern

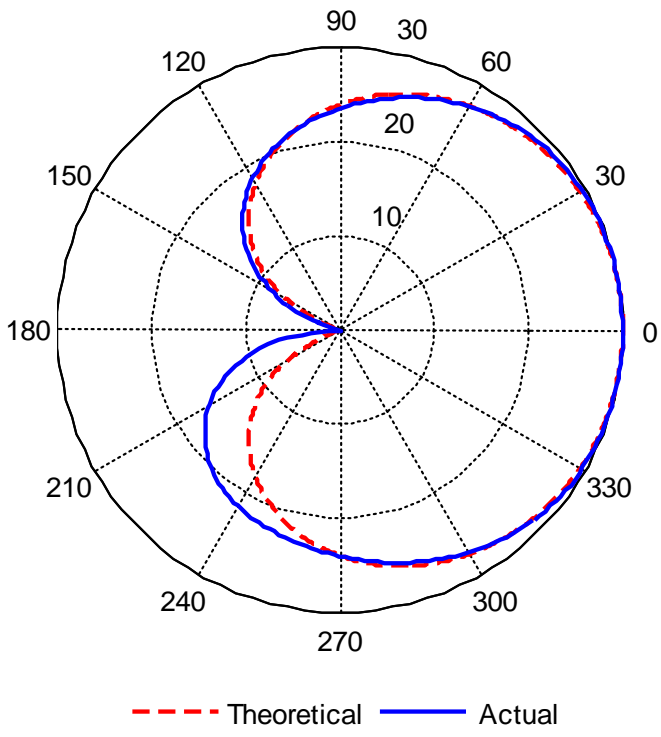

(c) Hydrophone cardioid beam pattern

Figure 35 Hydrophone actual vs. theoretical 2-D beam patterns in the vertical (xz) plane 
The actual plots in Figure 35(a)-(c) are not identical to the theoretical plots but are close. It appears that the asymmetry of the actual beam patterns is the result of the hydrophone not being rotated precisely about its central axis. There is evidence of this in the voltage magnitude plot in Figure 34: the dip in all four magnitudes near 215 degrees, the increase in all four magnitudes near 325 degrees, and the wide variation in the quadrant 2 magnitudes all indicate that the hydrophone was not rotated exactly about its central axis.

From the beam patterns, the actual 2-D vertical omni, dipole and cardioid directivity indexes were calculated using Equation (19), and the respective beam widths were measured. Table 11 displays the actual values next to the theoretical values. As with the beam patterns, the actual directivity indexes and beam widths are very close to the respective theoretical values.

Table 11 Hydrophone actual vs. theoretical vertical directivity indexes and beam widths

\begin{tabular}{ccccc}
\hline \hline & \multicolumn{2}{c}{ Theoretical } & \multicolumn{2}{c}{ Actual } \\
Pattern & 2-D DI & Beam Width & 2-D DI & Beam Width \\
\hline Omni & $0.0 \mathrm{~dB}$ & --- & $0.7 \mathrm{~dB}$ & --- \\
Dipole & $3.0 \mathrm{~dB}$ & 90.0 deg. & $3.2 \mathrm{~dB}$ & $93.0 \mathrm{deg}$. \\
Cardioid & $4.3 \mathrm{~dB}$ & 131.8 deg. & $4.1 \mathrm{~dB}$ & $129.0 \mathrm{deg}$. \\
\hline \hline
\end{tabular}

\subsection{Horizontal Directivity}

From the voltage time series data that was recorded for each quadrant while the hydrophone was rotated in its horizontal orientation, the test set calculated the complex voltage (magnitudes and phase) for each quadrant, at each angle of arrival. The plan had been to process the horizontal measurements in the same manner as the 
vertical measurements. However, when the horizontal measurements were taken, the hydrophone was not oriented so that the line separating quadrant 1 and quadrant 2 was facing the transmitter. That particular orientation - where the elevation angle theta $\theta$ equals 90 degrees - is the orientation of the theoretical beam pattern, and the only orientation that results in the horizontal cardioid beam pattern.

Therefore, the only horizontal actual vs. theoretical beam pattern comparison that can be made is the omnidirectional beam pattern comparison. This comparison is shown in Figure 36.

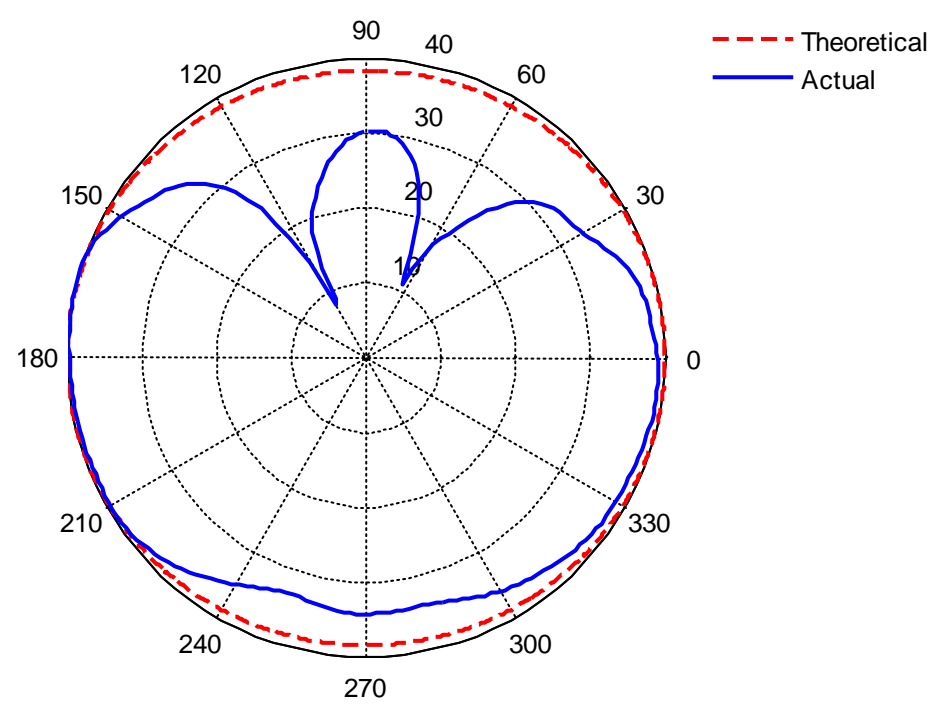

Figure 36 Hydrophone actual vs. theoretical 2-D beam pattern in the horizontal (xy) plane

From Figure 36, it can be seen that the actual horizontal beam pattern does not match the theoretical beam pattern. However, a probable explanation for the nulls that occur at roughly 60 and 120 degrees is that they were caused by interference from the fixture (shown in Figure 32). Furthermore, the arrival angles that matter are the broadside angles of 0 degrees and 180 degrees, and the values at these angles match almost exactly. 


\subsection{Conclusion}

Based upon the minimal differences between the theoretical and actual (vertical and horizontal) prototype beam patterns, directivity indexes and beam widths, it has been determined that this prototype served its intended purpose and demonstrated that cardioid receive beam patterns could be generated by a single four-quadrant multimode cylinder. 


\section{CHAPTER 9 ARRAY FABRICATION}

Next, the 15-cylinder linear hydrophone array was fabricated. Table 12 contains the material list.

Table 12 Array Material List

\begin{tabular}{cl}
\hline \hline Quantity & Description \\
\hline 15 & STEMiNC Piezoelectric ceramic (modified PZT-4) striped \\
& 4-quadrant cylinder, 42 kHz, radially poled, 26 mm OD x 22 \\
mm ID x 13 mm length, part number SMC2622T13111SP \\
15 & Gum rubber rings, 30mm OD x 26 mm ID x 9.5 mm length \\
1 & Array housing with 1.5” diameter brass cylinder endcaps \\
& and 6 - 1/8” diameter steel rods \\
15 feet & 24 AWG stranded bare wire \\
1 & 5-pin connector \\
3 feet & Clear rubber tubing, 1.5” ID x 1.875” OD \\
4 & Stainless steel hose clamps \\
6 inch & 2.0” diameter Delrin plastic rod \\
4 & Cable ties \\
& Heat-shrink plastic tubing \\
& Castor oil \\
& Solder \\
& Soldering flux \\
& Loctite 420 instant adhesive \\
\hline \hline
\end{tabular}

The array was constructed in the following manner in the order described:

The rubber rings were glued to the outside of the cylinders with a single small amount of instant adhesive. One of the six steel rods was removed from the frame so that the cylinders could be inserted. One of the remaining five steel rods was marked every 3.5 centimeters for placement of the cylinders. One at a time, each cylinder was placed within the steel rods and lined up with the appropriate placement mark. A drop of instant adhesive was used to secure the rubber ring to one of the steel rods in order 
to prevent the cylinder from moving. The 4 signal wires were pulled through the cylinder and connected to the appropriate inside quadrant with two solder dots.

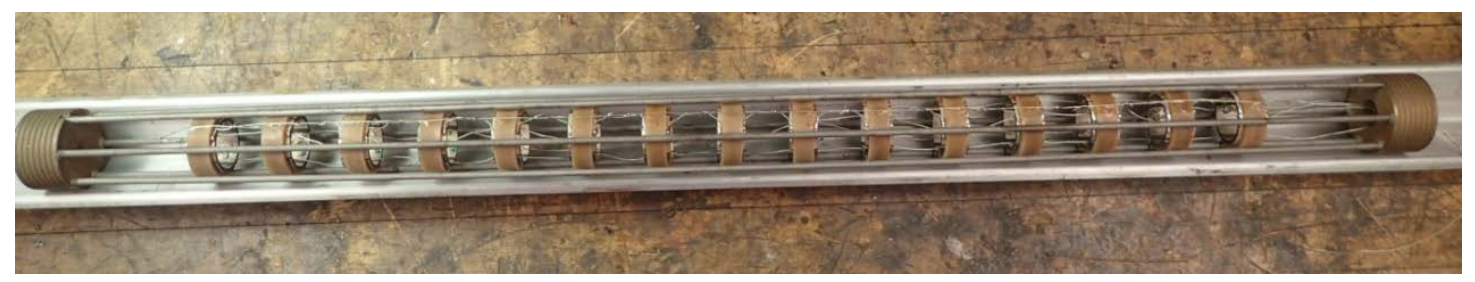

Figure 37 Photo 1 of array fabrication

After the fifteen cylinders were positioned and wired, the single ground wire was extended from cylinder 1 to cylinder 15 , and a small amount of adhesive was used to glue the wire to the outside of each rubber ring. The ground wire was then connected to the outside of each cylinder in two places (for redundancy) by two small wires.

The sixth steel rod was re-inserted into the frame, and three cable ties were wrapped around the steel rods to hold the rods securely against the rubber rings. A small amount of instant adhesive was used to glue the five remaining steel rods to the rubber rings.

Figures 37 and 38 are photos of the array during fabrication.

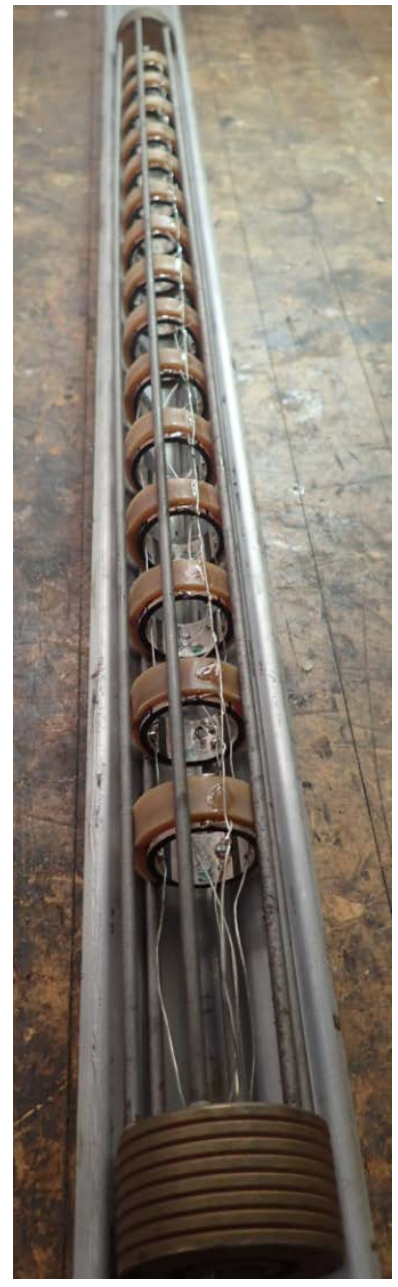

Figure 38 Photo 2 of array fabrication 
The five wires were cut so that they were long enough to extend just beyond the brass cylinder at the end of the frame. Heat shrink tubing was attached to the last few inches of the wires to prevent the bare wires from touching each other or the frame. These now shielded wires were brought through the hole in the brass cylinder at the end of the frame. The wires of the 5-pin connector were brought through the single hole in the first Delrin plug and the 5-pin connector was screwed into the plug. The 5 wires from the array were then soldered to the 5 wires of the 5-pin connector, and the solder joints were covered with additional heat shrink tubing (Figure 39).

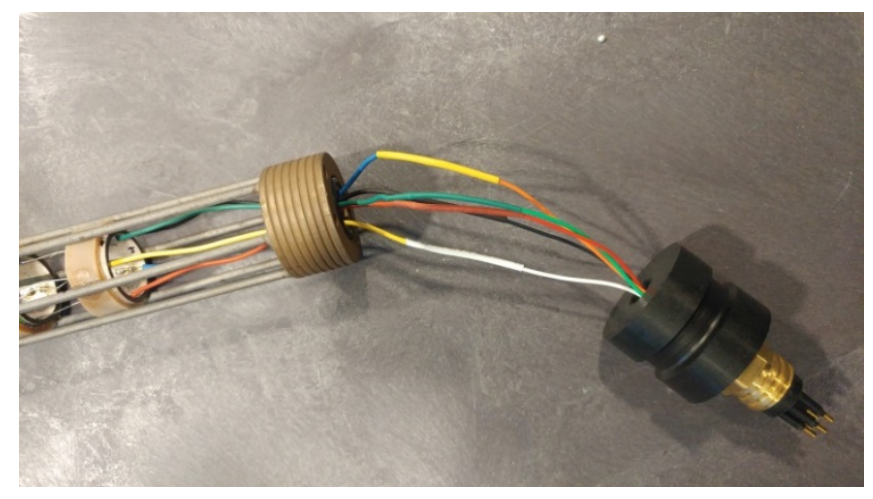

Figure 39 Photo 3 of array fabrication

The above assembly was slid into the rubber tubing (referred to as a boot). One clamp was placed around the rubber boot at the location of the brass cylinder and tightened. A second clamp was placed around the rubber boot at the location of the Delrin plug and tightened.

The booted array was filled with castor oil through the hole in the brass cylinder at the end opposite the 5-pin connector. When the oil had reached the brass cylinder, a second Delrin plug, this one with two small holes drilled through it, was inserted into the rubber boot about an inch above the brass cylinder. Again, one clamp was placed around the rubber boot at the location of the brass cylinder and tightened, and a second 
clamp was placed around the rubber boot at the location of the Delrin plug and tightened.

The area between the brass cylinder and the second Delrin plug was filled with castor oil through one of the holes in the plug - the other hole allowed the air to escape. Sealing screws were used to seal the two holes.

Figure 40 is a photo of the final 15-cylinder multimode linear hydrophone array.

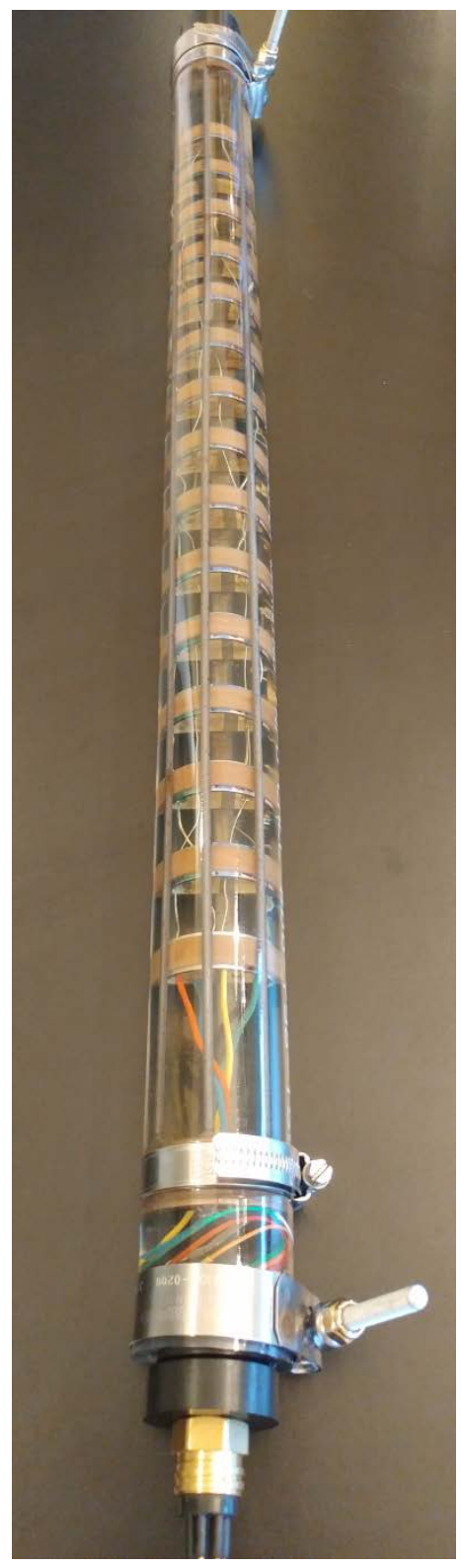

Figure 40 Photo of final array 


\section{CHAPTER 10 ARRAY TESTING}

On March 12, 2018, the multimode linear hydrophone array was tested in the Open Tank Facility (OTF) at the Naval Undersea Warfare Center (NUWC), Newport, RI - the same facility where the single-cylinder hydrophone prototype had been tested. The same test equipment was used.

This time the distance between the transmitter and array was increased from 2 meters to 6 meters to ensure far-field plane wave propagation. The following equation for the minimum separation distance for far-field water tank measurements for a linear array (Bobber, 1991) was used to determine the required separation:

$$
\mathrm{d} \geq \frac{\mathrm{L}^{2}}{\lambda}=\frac{.49^{2}}{.04}=6.0
$$

where

$d$ is the distance between the transmitter and the acoustic center of the array $L$ is the length of the linear array

$\lambda=c / f$ is the wavelength

$c$ is the sound speed

$f$ is the operating frequency

The same cable assembly that had been used to test the single-cylinder hydrophone was used, but because the array was now further from the transmitter, a 1.2 meter, a $1.6 \mathrm{~m}$ and a 1.8 meter RG-58 cable extension had to be added to each of the four cables.

The same tests that were previously run for the single-cylinder hydrophone prototype were repeated for the array. The test conditions are described in Table 13. 
Table 13 Test Conditions March 12, 2018

\begin{tabular}{ll}
\hline Parameter & Value \\
\hline $\begin{array}{l}\text { Separation distance between } \\
\text { projector and hydrophone }\end{array}$ & 6 meters \\
$\begin{array}{l}\text { Depth of projector and } \\
\text { hydrophone }\end{array}$ & $2.28 \mathrm{~m}$ (90 inches) \\
Transmit frequency & $\begin{array}{l}37.5 \mathrm{kHz} \text { (the designed operating } \\
\text { frequency of the hydrophone) }\end{array}$ \\
Water temperature & 18.8 degrees C \\
Sound speed & $1479 \mathrm{~m} / \mathrm{s}$ \\
Horizontal resolution & $0.25 \mathrm{degrees}$ \\
Vertical resolution & $0.50 \mathrm{degrees}$ \\
Sampling rate & $5 \mathrm{MHz}$ \\
Pulse duration & $0.50 \mathrm{~ms}$ \\
\hline \hline
\end{tabular}

For the vertical tests, a $2.5 \mathrm{lb}$. weight was attached to the bottom of the array to help keep it aligned. For the horizontal tests, the fixture shown in Figure 41 was used.

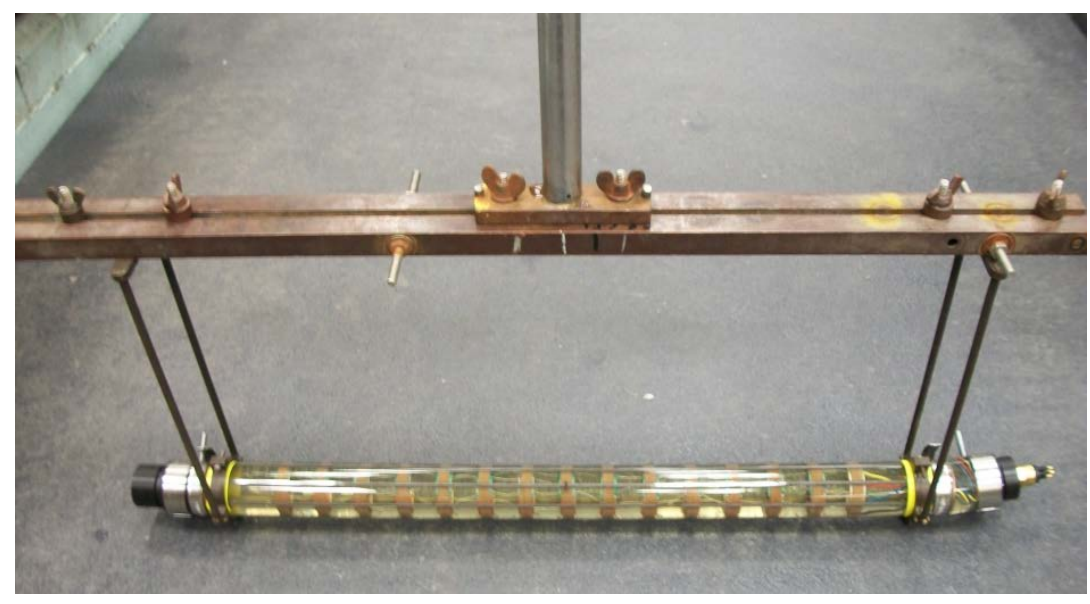

Figure 41 Array Horizontal Test Fixture 


\section{CHAPTER 11 PROCESSING THE ARRAY TEST RESULTS}

In this chapter, a quadrant refers collectively to the particular quadrant of all 15 cylinders, and a quadrant's data originates from the single wire attached to the particular quadrant of all 15 cylinders.

\subsection{Free Field Voltage Sensitivity}

As with the single-cylinder hydrophone, free field voltage sensitivity (FFVS) magnitudes were provided by the test set for each of the four quadrants over a range of frequencies. Figure 42 contains a plot of the FFVS magnitudes for each quadrant for frequencies from $10 \mathrm{kHz}$ to $50 \mathrm{kHz}$.

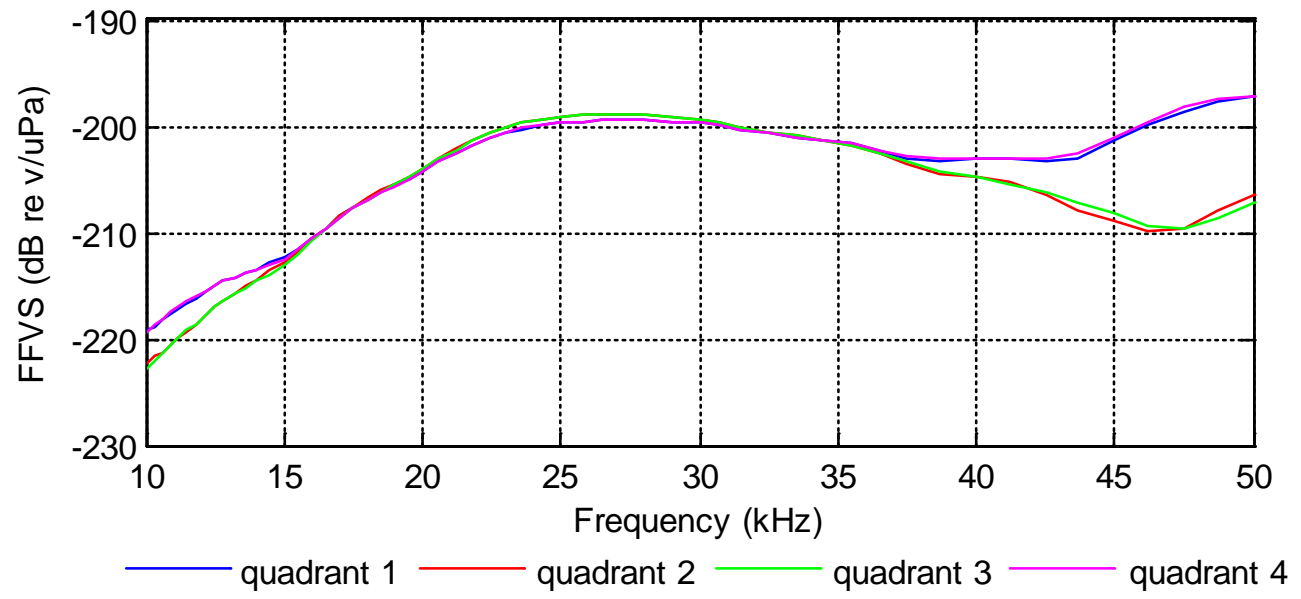

Figure 42 FFVS magnitude vs. frequency for hydrophone array

Table 14 lists the FFVS magnitudes that were calculated for each of the array's four quadrants at the operating frequency of $37.5 \mathrm{kHz}$. 
Table 14 Array FFVS Magnitude at $37.5 \mathrm{kHz}$

\begin{tabular}{cc}
\hline \hline Quadrant & $\begin{array}{c}\text { FFVS @ } 37.5 \mathrm{kHz} \\
(\mathrm{dB} \text { re } 1 \mathrm{~V} / \mu \mathrm{Pa})\end{array}$ \\
\hline 1 & -203.0 \\
2 & -203.5 \\
3 & -203.4 \\
4 & -202.8 \\
\hline \hline
\end{tabular}

\subsection{Vertical Directivity}

From the voltage time series data that was recorded for each quadrant while the array was rotated in its vertical orientation, the test set calculated the complex voltage (magnitudes and phase) for each quadrant, at each angle of arrival. Figure 43 shows how the voltage magnitudes and phases varied from one quadrant to the next as the array was rotated.
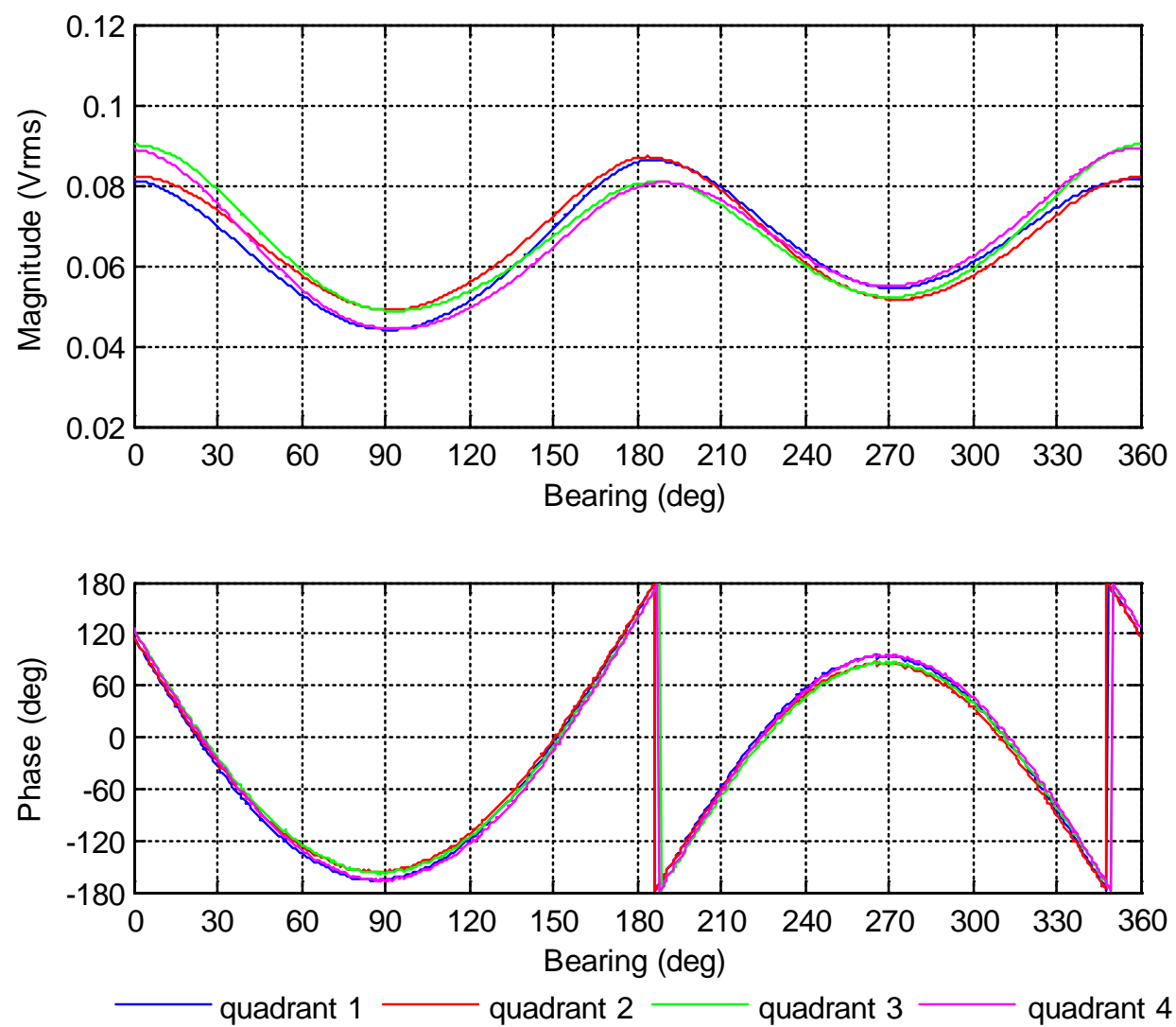

Figure 43 Array voltage magnitudes and phases at each angle in the vertical (xz) plane 
Again, Equations (26) through (34) were used to calculate the actual omni, dipole and cardioid beam patterns for this vertical rotation. The values used in Equation (31), and the resulting value for $R$, are shown here:

$$
\mathrm{R}=\frac{-\mathrm{V}_{\mathrm{O}}(\pi)}{\mathrm{V}_{\mathrm{D}}(\pi)}=\frac{-(-0.2819+0.1753 i)}{-0.0241-0.0145 i}=-5.3615+10.4939 \mathrm{i}
$$

The actual (measured) omni, dipole and cardioid beam patterns for this vertical rotation are compared to the theoretical (predicted) beam patterns in Figure 44(a)-(c).

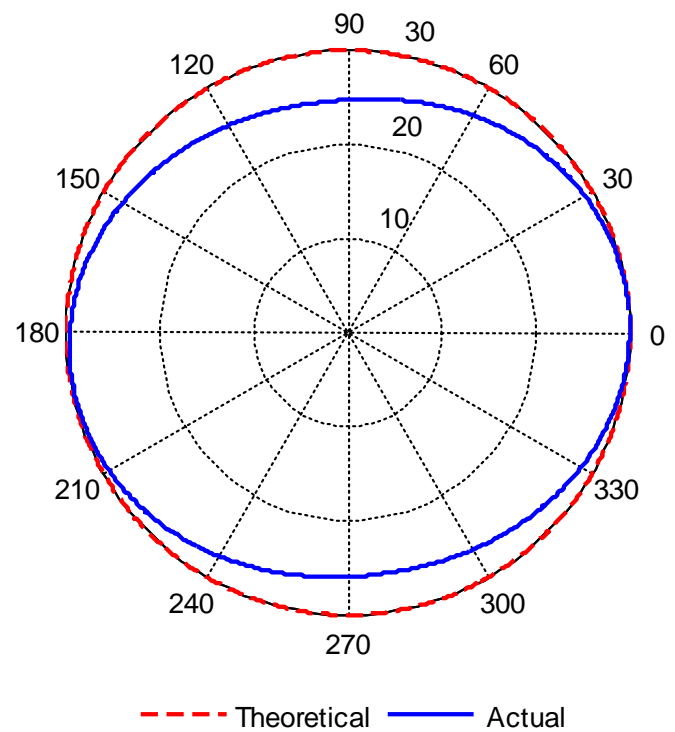

(a) Array omni beam pattern

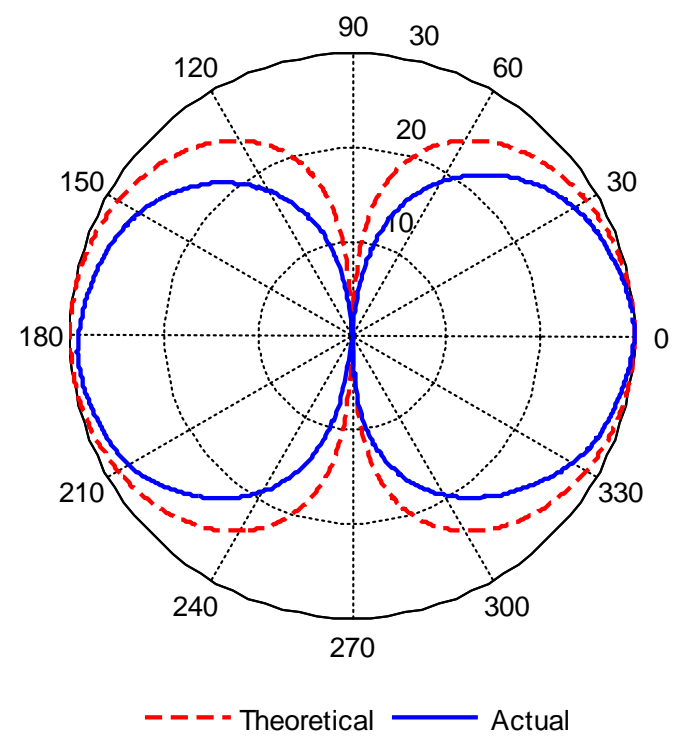

(b) Array dipole beam pattern

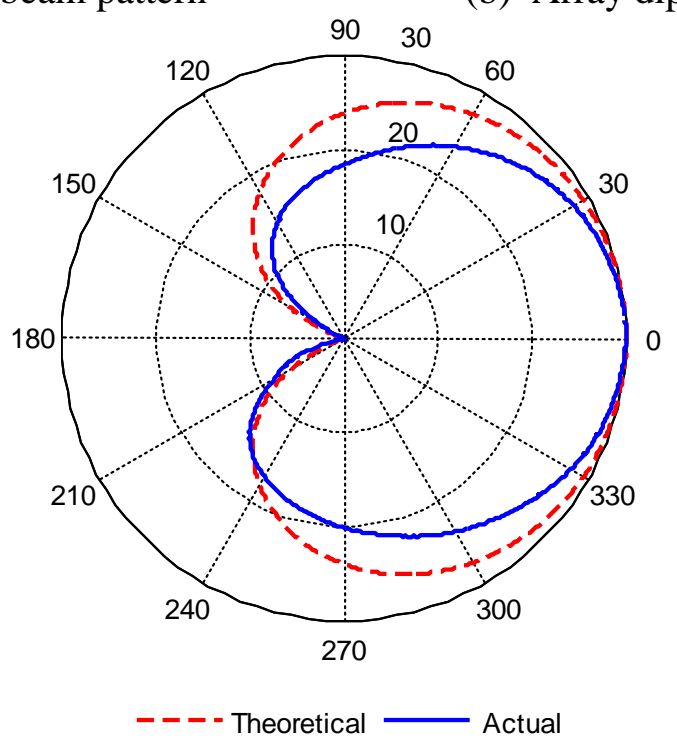

(c) Array cardioid beam pattern

Figure 44 Array actual vs. theoretical 2-D beam patterns in the vertical (xz) plane 
As with the single-cylinder hydrophone, the actual plots in Figure 44(a)-(c) are not identical to the theoretical plots, but share a strong resemblance. And again, it appears that the difference is due to the array not being rotated precisely about its central axis. There is evidence of this in the voltage magnitude plot in Figure 43 - at 90 and 270 degrees, all quadrants have a much reduced magnitude. This means that at those rotational angles the array was slightly further from the transmitter. These reduced magnitudes result in reduced magnitudes at 90 and 270 degrees in the beam pattern plots in Figure 44(a)-(c). Had the array been rotated precisely around its central axis, the actual and theoretical beam patterns would have likely been an excellent match.

From the beam patterns, the actual 2-D vertical omni, dipole and cardioid directivity indexes were calculated using Equation (19), and the respective beam widths were measured. Table 15 displays the actual values next to the theoretical values. Because of the reduced beam pattern magnitudes at 90 and 270 degrees, the actual directivity indexes and beam widths end up being much better than the respective theoretical values. Had the array been rotated precisely around its central axis, the actual and theoretical directivity indexes and beam widths would have likely been extremely close.

Table 15 Hydrophone actual vs. theoretical vertical directivity indexes and beam widths

\begin{tabular}{ccccc}
\hline \hline & \multicolumn{2}{c}{ Theoretical } & \multicolumn{2}{c}{ Actual } \\
Pattern & 2-D DI & Beam Width & 2-D DI & Beam Width \\
\hline Omni & $0.0 \mathrm{~dB}$ & --- & $2.1 \mathrm{~dB}$ & --- \\
Dipole & $3.0 \mathrm{~dB}$ & $90.0 \mathrm{deg}$. & $4.7 \mathrm{~dB}$ & $62.0 \mathrm{deg}$. \\
Cardioid & $4.3 \mathrm{~dB}$ & $131.8 \mathrm{deg}$. & $6.1 \mathrm{~dB}$ & $75.6 \mathrm{deg}$. \\
\hline \hline
\end{tabular}




\subsection{Horizontal Directivity}

From the voltage time series data that was recorded for each quadrant while the array was rotated in its horizontal orientation, the test set calculated the complex voltage (magnitudes and phase) for each quadrant, at each angle of arrival. As with the horizontal measurements of the single-cylinder hydrophone, when the horizontal array measurements were taken, the array was not oriented so that the line separating quadrant 1 and quadrant 2 was facing the transmitter (again, the orientation of the theoretical beam pattern, and the only orientation that results in the horizontal cardioid beam pattern). So, again, the only horizontal actual vs. theoretical beam pattern comparison that can be made is the omnidirectional beam pattern comparison. This comparison is shown in Figure 45.

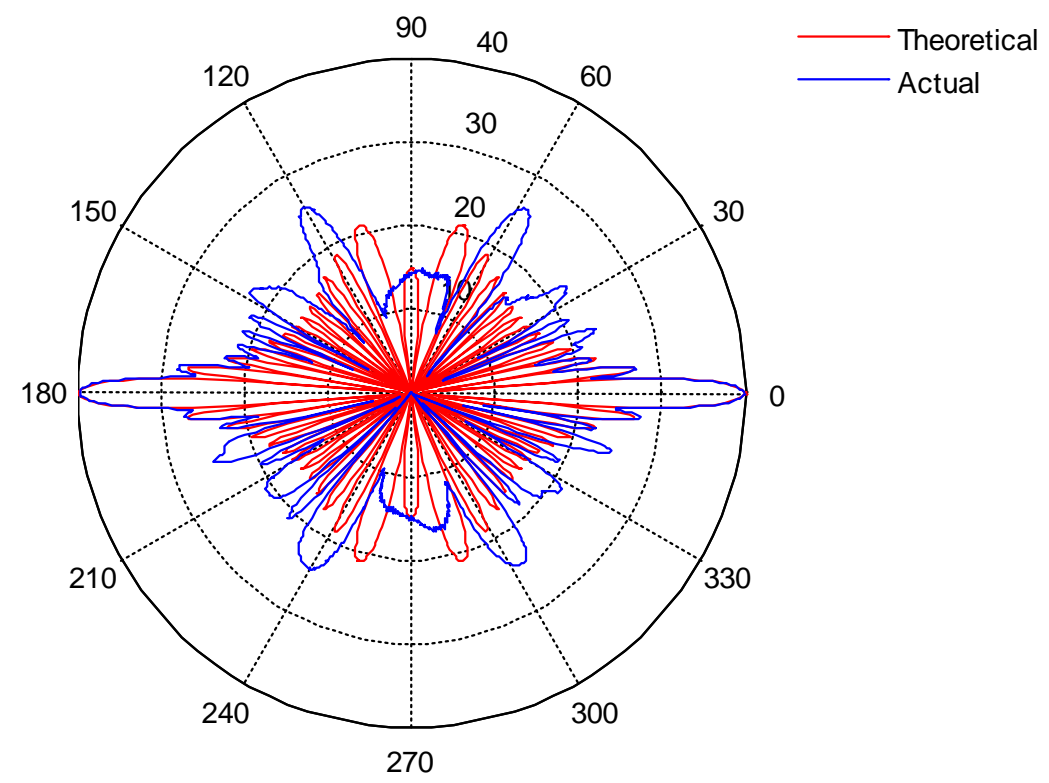

Figure 45 Array actual vs. theoretical 2-D beam pattern in the horizontal (xy) plane

The beam patterns are not a perfect match, but there is nothing about the actual beam pattern that indicates a problem. The following quantitative comparison 
between theoretical and actual directivity indexes and beam widths provides more conclusive information.

From the actual horizontal beam pattern, the actual directivity index was calculated using Equation (18), and the beam width was measured. Table 16 displays the actual values next to the theoretical values. This data shows that the actual 2-D horizontal directivity index is very close to the theoretical ( $15.0 \mathrm{~dB}$ vs. $15.8 \mathrm{~dB})$ and that the actual horizontal beam width is slightly better than the theoretical ( 3.8 degrees vs. 4.3 degrees).

Table 16 Actual vs. theoretical horizontal directivity indexes and beam widths

\begin{tabular}{cccc}
\hline \multicolumn{2}{c}{ Theoretical } & \multicolumn{2}{c}{ Actual } \\
2-D DI & Beam Width & 2-D DI & Beam Width \\
\hline $15.8 \mathrm{~dB}$ & $4.3 \mathrm{deg}$. & $15.0 \mathrm{~dB}$ & $3.8 \mathrm{deg}$. \\
\hline
\end{tabular}

\subsection{Conclusion}

The minimal differences between the theoretical and actual (vertical and horizontal) 2-D beam patterns, directivity indexes and beam widths, justify the usage of the array's theoretical 3-D Directivity Index (DI) of $18.35 \mathrm{~dB}$ (calculated in Chapter 4) when estimating the detection range $(r)$ of the array. 


\section{CHAPTER 12 SONAR EQUATION ANALYSIS}

Based upon 1) what is known about the existing TPL-25 system, and 2) the predicted Directivity Index (DI) of the 15-cylinder multimode linear hydrophone array (hereafter referred to as the proposed design), this chapter estimates the detection range $(r)$ for each, and compare the results. This chapter is organized as follows:

Section 12.1: Specifications and Assumptions

Section 12.2: Performance Analysis of Existing TPL-25 System

1. Estimate Transmission Loss (TL)

2. Estimate Directivity Index (DI)

3. Estimate Detection Threshold (DT)

4. Estimate Figure of Merit (FOM)

5. Estimate detection range $(r)$

Section 12.3: Performance Analysis of Proposed Design (multimode linear hydrophone array)

1. Use Transmission Loss (TL) from Section 12.2

2. Use Directivity Index (DI) from Chapter 11

3. Use Detection Threshold (DT) from Section 12.2

4. Estimate Figure of Merit (FOM)

5. Estimate detection range $(r)$

Section 12.4: Comparison of Proposed Design to Existing TPL-25 System

\subsection{Specifications and Assumptions}

The following details are based on Benthos and Dukane emergency beacon specifications (BEA, 2011): 
Beacon center frequency $=37.5 \mathrm{kHz}$

Source Level $(S L)=160 \mathrm{~dB}$

Signal duration $(t)=.01$ second

The following assumptions were made regarding the environment:

Ambient noise limited

Sea State $=3$

Salinity $=35 \mathrm{ppt}$

$\mathrm{pH}=8$

Water temperature $=4^{\circ} \mathrm{C}\left(39^{\circ} \mathrm{F}\right)$

Ideal volume noise model

The following assumptions were made regarding the system:

Desired Probability of Detection $p(D)=0.5$ (50\% probability of detection)

Desired Probability of False Alarm $p(F A)=0.0001$ ( 1 chance in $10^{4}$ of a false alarm occurring in the signal duration)

Integration time $(T)=.5$ seconds

Search depth $(d)=9843$ feet (3000 meters) (required for calculation of absorption coefficient $\alpha$ )

\subsection{Performance Analysis of Existing TPL-25 System}

The below ambient noise limited passive sonar equation was used to analyze the performance of the system (Urick p. 22, 1983):

$$
\mathrm{SL}-\mathrm{TL}=\mathrm{NL}-\mathrm{DI}+\mathrm{DT}
$$

\section{Source Level (SL)}

Source Level (SL) was given to be $160 \mathrm{~dB}$. 


\section{Noise Level (NL)}

From the Urick fig. 7.5 (1983) Wenz curve, given frequency of $37.5 \mathrm{kHz}$ and Sea State of 3, the Ambient Noise Level $(N L)$ is determined to be $35 \mathrm{~dB}$.

\section{Transmission Loss (TL)}

From Urick fig. 5.5 (1983), the absorption coefficient at zero depth $\alpha_{0}$ for the given frequency of $37.5 \mathrm{kHz}$, water temperature of $4^{\circ} \mathrm{C}$, salinity of $35 \mathrm{ppt}$, and $\mathrm{pH}$ of 8 is determined to be $8 \mathrm{~dB} / \mathrm{kyd}$. When $\alpha_{0}$ of $8 \mathrm{~dB} / \mathrm{kyd}$ and search depth $d$ of 9843 feet (3000 meters) are plugged into the following equation (Urick p. 108, 1983), the absorption coefficient $\alpha$ is determined to be $6.5 \mathrm{~dB} / \mathrm{kyd}$.

$$
\alpha=\alpha_{0}\left(1-1.93 \times 10^{-5} d\right)
$$

Transmission Loss (TL) for ranges $r$ between 0 and 10,000 yards is defined by Urick (p. 111, 1983) to be the following:

$$
\mathrm{TL}(r)=20 \log r+\alpha \mathrm{r} \times 10^{-3}
$$

\section{Directivity Index (DI)}

Given that the single hydrophone used in the TPL 25 is omnidirectional (Sasse, 2014), the beam pattern is given by:

$$
\mathrm{b}(\varnothing, \theta)=1
$$

When the beam pattern is plugged into Equation (16), the resulting Directivity Index $(D I)$ is $0 \mathrm{~dB}$.

\section{Detection Threshold (DT)}

From the Urick fig. 12.7 (1983) ROC curve, given desired Probability of Detection of 50\% and desired Probability of False Alarm of .01\%, the detection index, $d$, is determined to be 16. For the case of known signal wave shape (frequency, time 
of occurrence and duration), Urick (p. 394, 1983) gives the following equation for Detection Threshold (DT):

$$
\mathrm{DT}=10 \log \frac{d}{2 t}
$$

However, when the integration time is not "matched" to the signal duration, an additional term must be added to the equation as follows (Urick p. 394, 1983):

$$
\mathrm{DT}=10 \log \frac{d}{2 t}+\left|5 \log \frac{T}{t}\right|
$$

When detection index $(d)$, signal duration $(t)$ and integration time $(T)$ are plugged into Equation (45), Detection Threshold (DT) for the existing TPL-25 system is obtained:

$$
\mathrm{DT}=10 \log \frac{d}{2 t}+\left|5 \log \frac{T}{t}\right|=10 \log \frac{16}{2 \cdot .01}+\left|5 \log \frac{.5}{.01}\right|=37.5 d B
$$

\section{Figure of Merit (FOM)}

Figure of Merit $(F O M)$ is the amount of transmission loss that results in a signal excess of $0 \mathrm{~dB}$ (Hodges p. 20, 2010). FOM is therefore calculated by setting the signal excess to zero and solving the sonar equation for Transmission loss (Hodges p. 21, 2010). For a passive sonar system, FOM is defined by Urick (p. 23, 1983) as follows:

$$
\mathrm{FOM}=\mathrm{SL}-(\mathrm{NL}-\mathrm{DI}+\mathrm{DT})
$$

When Source Level $(S L)$, Noise Level $(N L)$, Directivity Index $(D I)$ and Detection Threshold (DT) are plugged into Equation (46), Figure of Merit (FOM) for the existing TPL-25 system is obtained:

$$
\mathrm{FOM}=\mathrm{SL}-(\mathrm{NL}-\mathrm{DI}+\mathrm{DT})=160-(35-0+37.5)=87.5
$$




\section{Detection Range ( $r$ )}

By knowing the FOM, one can look at a plot of Transmission Loss (TL) versus range and determine the maximum range at which a sonar can detect its target (Hodges p. 21, 2010) - the range where TL and FOM intersect. This is the detection range $(r)$.

Figure 46 contains a plot of FOM for the existing TPL-25 system and Transmission Loss (TL) versus range.

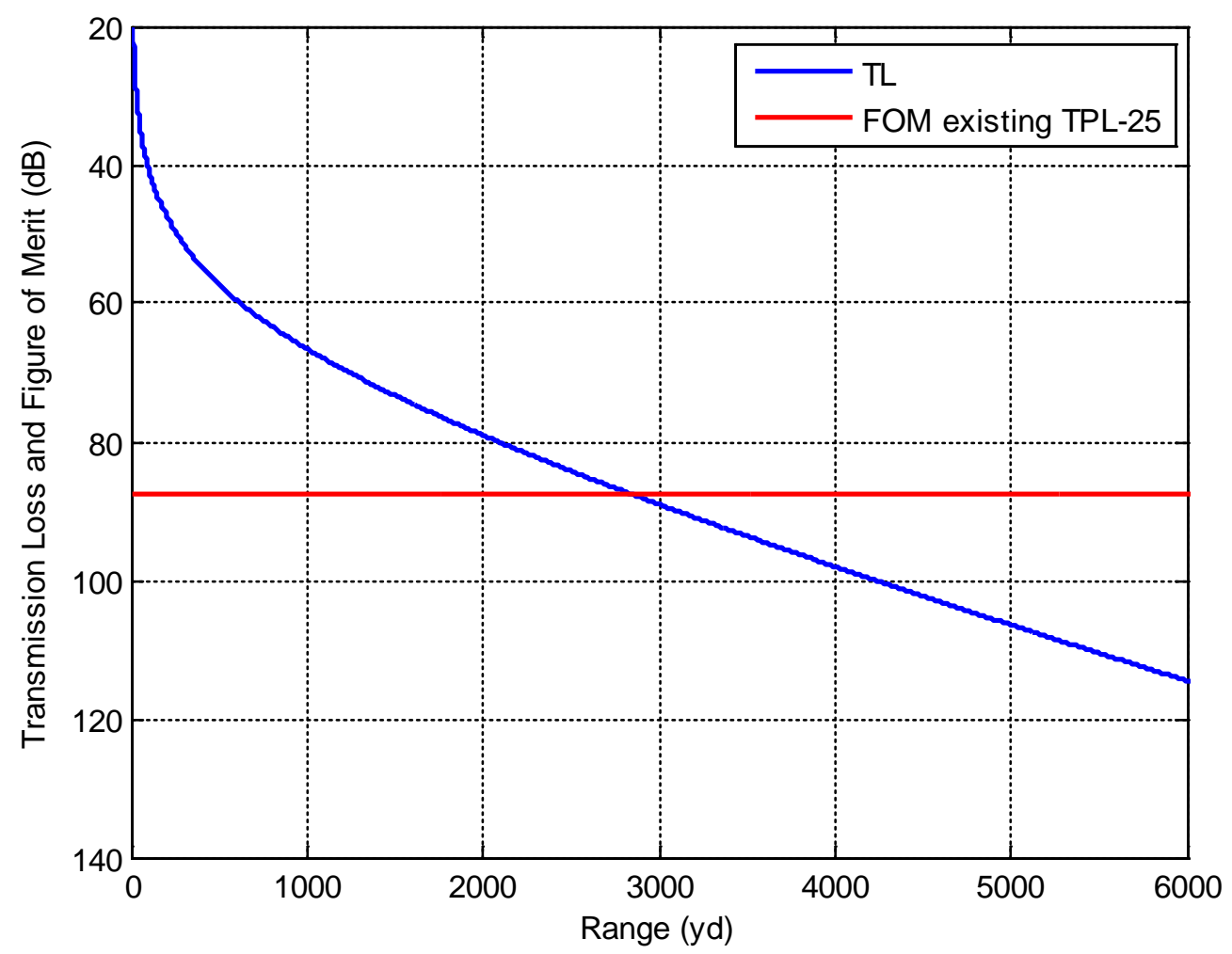

Figure 46 Transmission Loss and Figure of Merit of the Existing TPL-25 System

From the above plot, it can be determined that the detection range $(r)$ for the existing TPL-25 system is 2840 yards (2597 meters). 
Summary of Performance analysis for existing TPL-25 system

Table 17 contains a summary of the performance analysis for the existing TPL-25 system.

Table 17 Summary of existing TPL-25 system performance analysis

\begin{tabular}{lcc}
\hline \hline Parameter & Value & Source \\
\hline Beacon center frequency & $37.5 \mathrm{kHz}$ & given \\
Beacon Source Level $(S L)$ & $160.0 \mathrm{~dB}$ & given \\
Beacon signal duration $(t)$ & $0.01 \mathrm{sec}$ & given \\
Probability of Detection $p(D)$ & $50.00 \%$ & given \\
Probability of False Alarm $p(F A)$ & $0.01 \%$ & given \\
Integration Time $(T)$ & $0.5 \mathrm{sec}$ & given \\
Ambient Noise Level $(N L)$ & $35.0 \mathrm{~dB}$ & from Wenz curve \\
Detection index $(d)$ & 16 & from ROC curve \\
Absorption coefficient $(\alpha)$ & $6.5 \mathrm{~dB} / \mathrm{kyd}$ & Urick fig. 5.5/ \\
& & calculated \\
Directivity Index $(D I)$ & $0 \mathrm{~dB}$ & calculated \\
Detection Threshold $(D T)$ & $37.5 \mathrm{~dB}$ & calculated \\
Figure of Merit $(F O M)$ & $87.5 \mathrm{~dB}$ & calculated \\
Detection range $(r)$ & $2597 \mathrm{~m}$ & calculated \\
\hline
\end{tabular}

\subsection{Performance Analysis of Proposed Design (Multimode Linear Array)}

\section{Directivity Index (DI)}

Directivity Index $(D I)$ of $18.35 \mathrm{~dB}$ is being used for the 15-cylinder multimode linear hydrophone array (Section 11.4). 


\section{Figure of Merit (FOM)}

When Source Level $(S L)$, Noise Level $(N L)$, Directivity Index $(D I)$ and Detection Threshold (DT) are plugged into Equation (46), Figure of Merit (FOM) for the proposed design is obtained:

$$
\mathrm{FOM}=\mathrm{SL}-(\mathrm{NL}-\mathrm{DI}+\mathrm{DT})=160-(35-18.35+37.5)=105.8
$$

\section{Detection Range ( $r$ )}

Figure 47 contains a plot of FOM for the existing TPL-25 system (red), FOM for the proposed design (green), and Transmission Loss (TL) versus range.

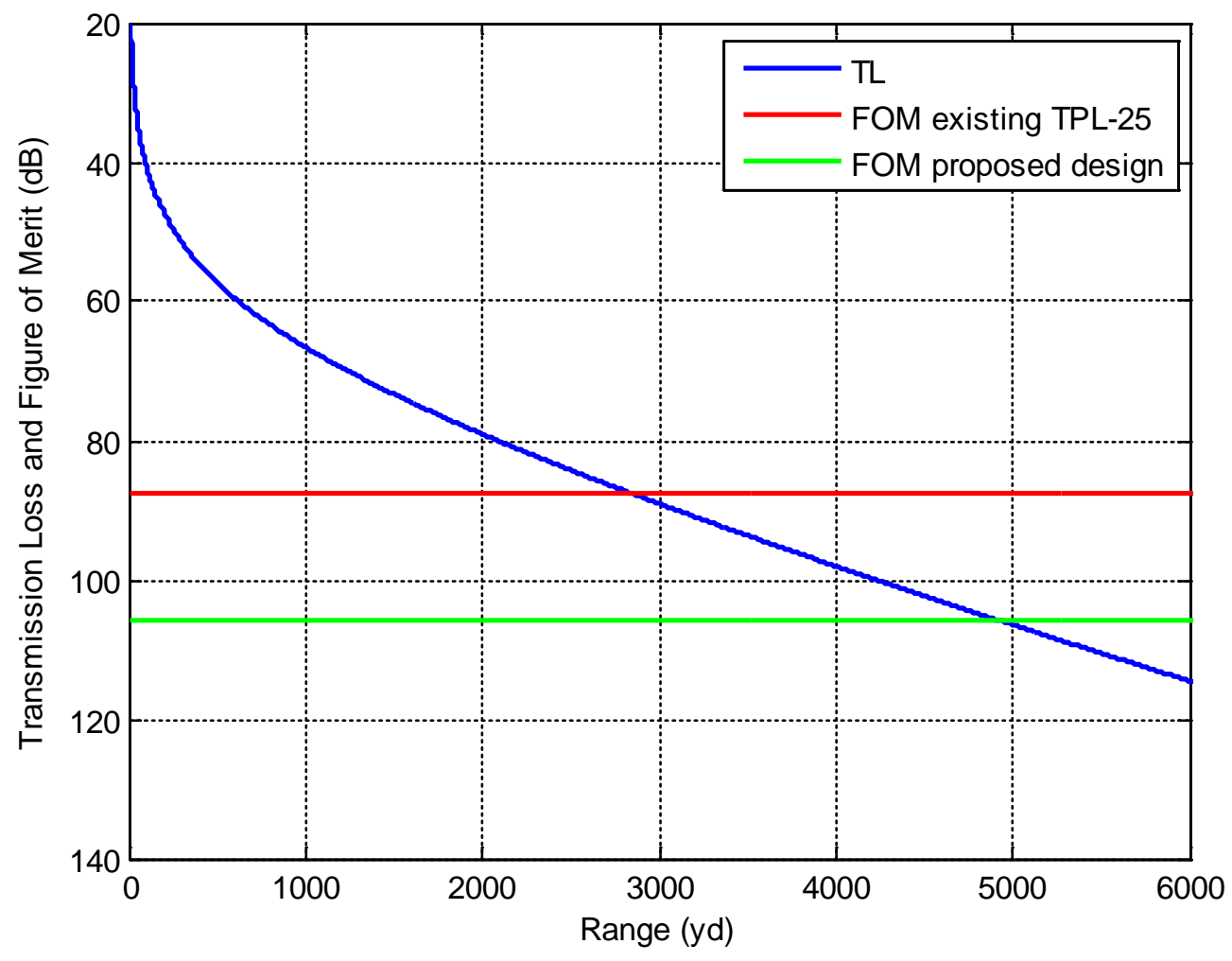

Figure 47 Transmission Loss and Figure of Merit of the Proposed Design

From the above plot, it can be determined that the detection range $(r)$ for the proposed design is 4932 yards (4510 meters). 
Summary of Performance analysis for proposed design

Table 18 contains a summary of the performance analysis for the proposed design.

Table 18 Summary of proposed design performance analysis

\begin{tabular}{lcc}
\hline Parameter & Value & Source \\
\hline Beacon center frequency & $37.5 \mathrm{kHz}$ & given \\
Beacon Source Level $(S L)$ & $160.0 \mathrm{~dB}$ & given \\
Beacon signal duration $(t)$ & $0.01 \mathrm{sec}$ & given \\
Probability of Detection $p(D)$ & $50.00 \%$ & given \\
Probability of False Alarm $p(F A)$ & $0.01 \%$ & given \\
Integration Time $(T)$ & $0.5 \mathrm{sec}$ & given \\
Ambient Noise Level $(N L)$ & $35.0 \mathrm{~dB}$ & from Wenz curve \\
Detection index $(d)$ & 16 & from ROC curve \\
Absorption coefficient $(\alpha)$ & $6.5 \mathrm{~dB} / \mathrm{kyd}$ & Urick fig. 5.5/ \\
& & calculated \\
Directivity Index $(D I)$ & $18.35 \mathrm{~dB}$ & calculated \\
Detection Threshold $(D T)$ & $37.5 \mathrm{~dB}$ & calculated \\
Figure of Merit $(F O M)$ & $105.8 \mathrm{~dB}$ & calculated \\
Detection range $(r)$ & $4510 \mathrm{~m}$ & calculated \\
\hline \hline
\end{tabular}

\subsection{Comparison of Proposed Design to Existing System}

Table 19 compares FOM and detection range $(r)$ between the existing TPL-25 system and the proposed design:

Table 19 Comparison of FOM and detection range

\begin{tabular}{lcc}
\hline \hline & Figure of Merit & Detection Range \\
\hline Existing TPL-25 System & $87.5 \mathrm{~dB}$ & $2597 \mathrm{~m}$ \\
Proposed Design & $105.8 \mathrm{~dB}$ & $4510 \mathrm{~m}$ \\
\hline
\end{tabular}




\section{CHAPTER 13 SUMMARY AND CONCLUSION}

\subsection{Summary}

This thesis explored how replacing the TPL-25's single omnidirectional hydrophone with a device that has an increased directivity index, specifically with a linear array of directional multimode hydrophones, could increase the detection range of the TPL-25.

The use of multimode technology as a means to increase the directionality of a transducer was researched and this research was presented in Chapter 2. This multimode technology was first applied to a single-cylinder hydrophone prototype. The theoretical directivity of the single-cylinder multimode hydrophone prototype was predicted (Chapter 4), and the prototype was fabricated (Chapter 6) and then tested in an acoustic test tank (Chapter 7). The data collected in the tank was processed with multimode signal processing, a cardioid beam pattern was successfully created, and the prototype's calculated horizontal and vertical directivity indexes were shown to be comparable to the predicted values (Chapter 8).

The multimode technology was then applied to a 15-cylinder linear hydrophone array. The array was designed (Chapter 3), and the theoretical directivity of the multimode linear hydrophone array was predicted (Chapter 4). The array was fabricated (Chapter 9), and then tested in the acoustic test tank (Chapter 10). The data collected in the tank was processed with multimode signal processing, and the calculated horizontal and vertical beam patterns and directivity indexes were shown to be comparable to those predicted (Chapter 11). 
The theoretical 3-D Directivity Index (DI) of the 15-cylinder multimode linear hydrophone array was calculated to be $18.35 \mathrm{~dB}$ (Chapter 4), and this DI was used in the passive sonar equation to determine the predicted Figure of Merit $(F O M)$ and detection range $(r)$ of the multimode linear hydrophone array (Chapter 12).

\subsection{Conclusion}

When the 15-cylinder multimode linear hydrophone array's theoretical Directivity Index $(D I)$ of $18.35 \mathrm{~dB}$ was plugged into the passive sonar equation, the Figure of Merit (FOM) was calculated to be $105.8 \mathrm{~dB}$ - this FOM is $18.3 \mathrm{~dB}$ higher than the FOM calculated for the current TPL-25 system.

The multimode array's detection range $(r)$, based upon this increased FOM, was calculated to be 4510 meters - this detection range is 1913 meters (73.7\%) greater than the detection range calculated for the current TPL-25 system.

This thesis, which researched, designed, fabricated and tested the application of multimode technology to a linear hydrophone array, concludes that a linear array of 15 multimode cylinders would be able to increase the detection range of the TPL-25 - the system currently used by the U.S. Navy to locate the black boxes from downed aircraft - by $73.7 \%$.

\subsection{Areas of Future Research}

One area of future research would be to calculate the Area Coverage Rate (ACR) for both the existing TPL-25 system and the multimode linear hydrophone array based upon the detection ranges and other relevant factors. 
Another area of future research would be to explore the use of the multimode technology (using the phase difference between the dipole response and the omnidirectional response) to identify the Direction of Arrival (DOA) of the emergency beacon's signal.

Still another area of future research would be to test the multimode linear hydrophone array in the ocean or bay with an actual emergency beacon. 


\section{LIST OF ACRONYMS AND ABBREVIATIONS}

2-D Two-Dimensional

3-D Three-Dimensional

ACR Area Coverage Rate

A/D Analog-to-Digital

AF $\quad$ Array Factor

BEA Bureau d'Enquêtes et d'Analyses pour la sécurité de l'aviation civile

(translated as "Bureau of Enquiry and Analysis for civil aviation safety")

dB Decibel

DI Directivity Index

DOA Direction of Arrival

FEM Finite Element Method

FFVS Free Field Voltage Sensitivity

ID Inside Diameter

LCR Inductance, Capacitance, and Resistance

MRA Main Response Axis

NAVSEA Naval Sea Systems Command

NUWC Naval Undersea Warfare Center

OD Outside Diameter

OTF Open Tank Facility

PZT Lead Zirconate Titanate

SNR Signal-to-Noise Ratio

STEMiNC Steiner \& Martins, Inc.

SUPSALV Supervisor of Salvage and Diving 
THAMES Transducer \& Hydrophone Acoustic Measurement \& Evaluation System

ULB Underwater Locator Beacon

USRD Underwater Sound Reference Division 


\section{BIBLIOGRAPHY}

Acoustical Society of America, AMERICAN NATIONAL STANDARD: Procedures for Calibration of Underwater Electroacoustic Transducers, ANSI/ASA S1.202012, Melville, NY, 2012.

Alvanas, E. A., Performance Analysis and Upgraded Design for Phoenix Towed Pinger Locator 25, University of Rhode Island OCE 472 Final Project, 13 December 2015.

Balanis, C. A., Antenna Theory: Analysis and Design, Third Edition, John Wiley \& Sons, Inc., Hoboken, New Jersey, 2005.

Bloomberg, Australia Narrows 'Pinger' Search for MH370 to Within Kilometers, gCaptain, April 2014, http:/gcaptain.com/australia-narrows-pinger-search-mh370within-kilometers/, accessed April 2018.

Bobber, R. J., Underwater Electroacoustic Measurements, Peninsula Publishing, Los Altos, CA, 1988.

Burdic, W. S., Underwater Acoustic System Analysis, 2nd Edition, Prentice Hall, Englewood Cliffs, NJ, 1991.

Bureau d'Enquêtes et d'Analyses (BEA) pour la sécurité de l'aviation civile (translated as "Bureau of Enquiry and Analysis for civil aviation safety"), $\underline{\text { Measurement of }}$ acoustic beacon mounted on flight recorders, Paris, November 2011.

Butler, J. L. and Butler, A. L., Multimode Synthesized Beam Transduction Apparatus, U.S. Patent 6,734,604 B2, May 2004.

Butler, J. L., Butler, A. L. and Rice, J. A., A tri-modal directional transducer, Journal of the Acoustical Society of America, Vol. 115, No. 2, pp. 658-665, February 2004. 
Butler, S. C., A directional dogbone flextensional transducer, Acoustical Society of America, Proceedings of Meetings on Acoustics, Vol. 11, 030001, 2010.

Butler, S. C., Butler, J. L., Butler, A. L. and Cavanagh, G. H., A low-frequency directional flextensional transducer and line array, Journal of the Acoustical Society of America, Vol. 102, No. 1, pp. 308-315, July 1997.

Ehrlich, S. L. and Frelich, P. D., Sonar Transducer, U.S. Patent 3,290,646, December 6, 1966.

Ellis, D. D., Effect of Cardioid and Limacon Directional Sensors on Towed Array Reverberation Response, Canadian Acoustics, Vol. 34, No. 3, 2006.

Hodges, R. P., Underwater Acoustics: Analysis, Design and Performance of Sonar, John Wiley \& Sons, Ltd., West Sussex, United Kingdom, 2010.

Hu, J. H., Li, H. L., Chan, H. L. W. and Choy, C. L., A ring-shaped piezoelectric transformer operating in the third symmetric extensional vibration mode, Elsevier Science Sensor and Actuators, Vol. 88, pp. 79-86, 2001.

Kinsler, L. E., Frey, A. R., Coppens, A. B. and Sanders, J. V., Fundamentals of Acoustics, Fourth Edition, John Wiley \& Sons, Inc., Hoboken, NJ, 2000.

Lamothe, D., Why the Navy's Black Box Locator Could Be Useless in the Hunt for Missing Flight 370, Foreign Policy (FP), March 2014, http://foreignpolicy.com/2014/03/24/why-the-navys-black-box-locator-could-beuseless-in-the-hunt-for-missing-flight-370/, accessed April 2018.

Li, H. L., Hu, J. H. and Chan, H. L. W., Finite Element Analysis on Piezoelectric Ring Transformer, IEEE Ultrasonics Symposium, 2002. 
Lim, Y., Joh, C., Seo, H. and Roh, Y., Design and Fabrication of a Multimode Ring Vector Hydrophone, Proceedings of Symposium on Ultrasonic Electronics, Vol. 34 (2013) pp. 131-132, 20-22 November, 2013.

McConnell, J. A., Haberman, R. C., McCormick, T. A. and Rudzinsky, R., Forming First- and Second-Order Cardioids with Multimode Hydrophones, The Journal of the Acoustical Society of America, 119, 3445 (2006).

Naval Sea Systems Command (NAVSEA), Ocean Search Assets, http://www.navsea.navy.mil/Home/SUPSALV/00C2-Salvage/Ocean-SearchAssets/, accessed April 2018.

Nuttall, A. H. and Cray, B. A., Approximations to Directivity for Linear, Planar, and Volumetric Apertures and Arrays, IEEE Journal of Oceanic Engineering, Vol. 26, No. 3, July 2001.

Paolero, A. E. and Évora, V. F., An Overview of the Underwater Sound Reference DivNpt (USRD), Presented to The National Institute of Standards and Technology, Gaithersburg, Maryland, 15 June 2015.

Sasse, R., NAVSEA 00C, SUPSALV Deep Ocean Search \& Recovery, Emerging Flight Data \& Locator Technology Forum, 7 October 2014.

Sherman, C. H. and Butler, J. L, Transducers and Arrays for Underwater Sound, Springer, New York, 2007.

Steiner \& Martins, Inc. (STEMiNC), https://www.steminc.com/PZT/en/, accessed April 2018.

Urick, R. J., Principles of Underwater Sound, Third Edition, Peninsula Publishing, Los Altos, CA, 1983. 
U.S. 7th Fleet Public Affairs, U.S. Navy Maps Downed C-2A Greyhound, February 2018, https://www.dvidshub.net/news/printable/266248, accessed April 2018. 